\title{
WestVirginiaUniversity
}

THE RESEARCH REPOSITORY @ WVU

Graduate Theses, Dissertations, and Problem Reports

2007

\section{Fabrication and functional analysis of SPARROW biosensor}

Praneetha Poloju
West Virginia University

Follow this and additional works at: https://researchrepository.wvu.edu/etd

\section{Recommended Citation}

Poloju, Praneetha, "Fabrication and functional analysis of SPARROW biosensor" (2007). Graduate Theses, Dissertations, and Problem Reports. 4328.

https://researchrepository.wvu.edu/etd/4328

This Thesis is protected by copyright and/or related rights. It has been brought to you by the The Research Repository @ WVU with permission from the rights-holder(s). You are free to use this Thesis in any way that is permitted by the copyright and related rights legislation that applies to your use. For other uses you must obtain permission from the rights-holder(s) directly, unless additional rights are indicated by a Creative Commons license in the record and/ or on the work itself. This Thesis has been accepted for inclusion in WVU Graduate Theses, Dissertations, and Problem Reports collection by an authorized administrator of The Research Repository @ WVU. For more information, please contact researchrepository@mail.wvu.edu. 


\title{
FABRICATION AND FUNCTIONAL ANALYSIS OF SPARROW BIOSENSOR
}

\author{
By \\ Praneetha Poloju

\begin{abstract}
Thesis Submitted to the
College of Engineering and Mineral Resources at

West Virginia University

in partial fulfillment of the requirements for the degree of
\end{abstract} \\ Master of Science \\ in \\ Electrical Engineering
}

Committee members:

Dr. Lawrence A. Hornak (Committee Chairperson)

Dr. Dimitris Korakakis

Dr. Xian A. Cao

Lane Department of Computer Science and Electrical Engineering

Morgantown, West Virginia

2007

Keywords: Electron Beam evaporation, SPARROW Biosensor, Biolayer, Flow cell experiments, Waveguide Characterization 


\section{Abstract \\ FABRICATION AND FUNCTIONAL ANALYSIS OF SPARROW BIOSENSOR}

\section{Praneetha Poloju}

Biosensors are powerful tools aimed at providing selective identification of toxic chemical compounds in industrial products, chemical substances, environmental samples or biological systems (e.g., bacteria, virus, or tissue components) for biomedical diagnosis. A new generation of biosensors are being developed, which combine the sensitivity and at the low cost of microelectronics.

The Nano/Micro Devices group at Microelectronics Systems Research Center (MSRC) has been researching stacked waveguide optical sensors useful for detecting and quantifying events in biological molecular chemistry, for example, binding events. This thesis describes the results of our initial research of (SPARROW) based technology. This study contains the design and implementation of a SPARROW biosensor with detailed disclosure of building the biosensor involving various steps such as Modeling, fabrication, optical measurements, waveguide loss calculations, simulation software for designing and analyzing the waveguide behavior and experiments to determine the sensors output for water and different concentrations of sucrose.

The SPARROW sensor is based on evanescent wave technique and can detect the changes in refractive index arising from the surface alterations. This architecture can operate as a biosensor through the change in the coupled optical power resulted from antigen bonding to the bio-layer. This thesis describes the process developed to optically characterize the alumina waveguides of the SPARROW device and investigates waveguide film quality as a function of fabrication parameters. These films were fabricated using Ion Source assisted Electron Beam Evaporator. Losses of 1-3 dB/cm have been measured for guiding films using the scattered power measurement technique. The optical binding of the microfluidic channel to the SPARROW device and results from the flow cell experiments are detailed.

This research has been published at the Fall 2006 MRS Conference sensors conference. 


\section{Acknowledgements}

First and foremost, I would like to thank and express my deep appreciation to Dr. Hornak, who has been more than a guide to me during this research. His valuable insight into all areas of the project and his invaluable moral support during the course of the research will never be forgotten.

I would like to thank Dr. Korakakis for his guidance and support. His inputs have been extremely valuable during the course of the thesis and his "sense of seriousness" made this project a fun-filled learning experience.

I would also like to thank Dr. Cao for serving on the advisory committee and for his invaluable inputs, Dr. Brown (Cleanroom Supervisor) for training me with the cleanroom equipment and helping me troubleshoot problems with the Ebeam evaporator and other equipment. Special thanks to my colleague, Joshua Ryan Nightingale and project team members Dr. Min Lim, Ke Feng and all my labmates. I enjoyed every moment working with them.

I thank the funding organization, Office of Naval Research for funding the project.

Thanks are also owed to my dear friends - Hiranmay, Abhilash, Dinesh and my other friends at WVU, whose continuous support made it much easier for me to finish this thesis.

Finally, I would like to express my gratitude and special thanks to my parents and siblings Karuna, Vasavi and Nikhil, Dr. Aminul Islam and his family for their love and encouragement, who were extremely supportive during the work. Without their support, this work would not have been a success. 


\section{Contents}

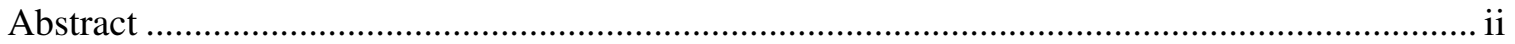

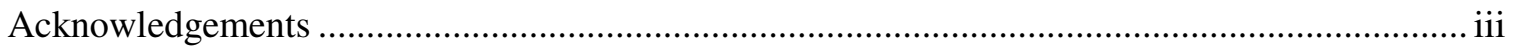

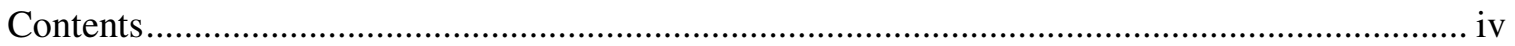

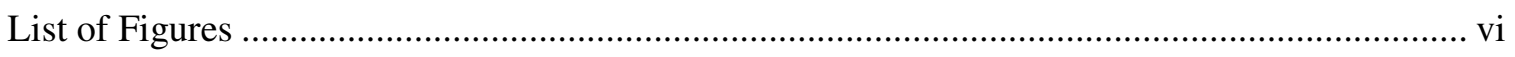

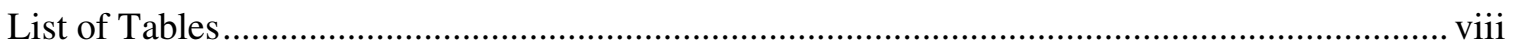

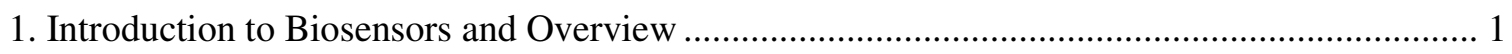

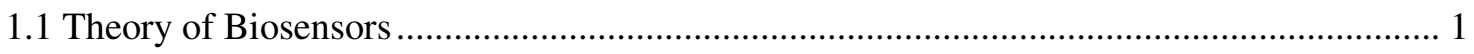

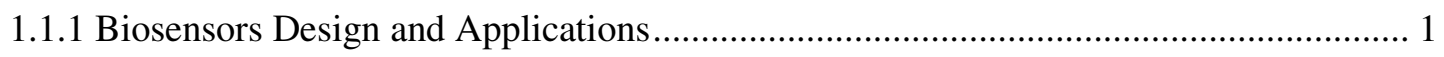

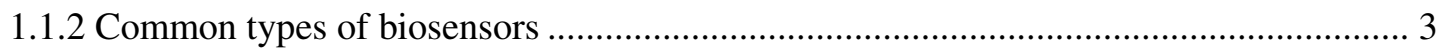

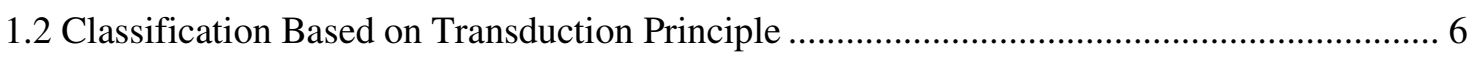

1.2.1. Chemomechanical or Mass-Sesnsitive sensors ........................................................... 7

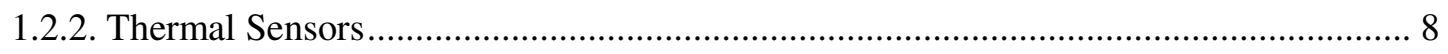

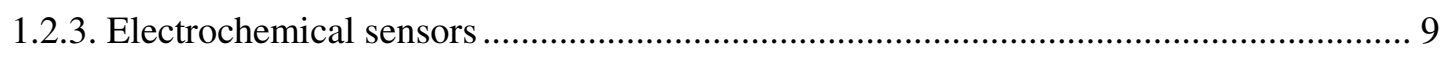

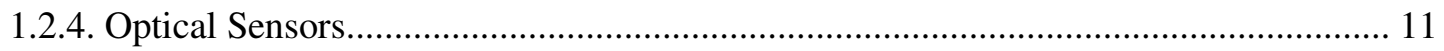

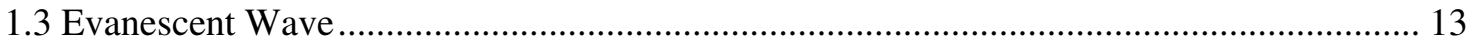

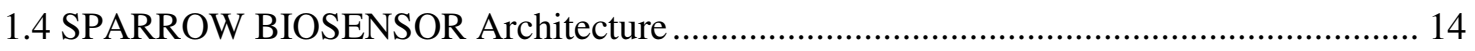

1.4.1 Fundamental Design of SPARROW Biosensor ....................................................... 14

1.4.2 Principle of Operation of SPARROW Biosensor........................................................ 15

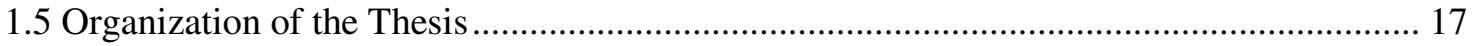

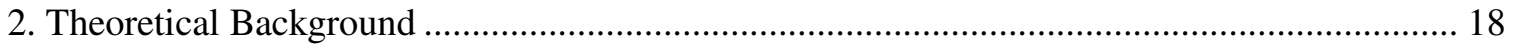

2.1 Basics of Electromagnetic Theory and Dielectric waveguides......................................... 18

2.1.1 Boundary Conditions at Dielectric Interfaces .......................................................... 19

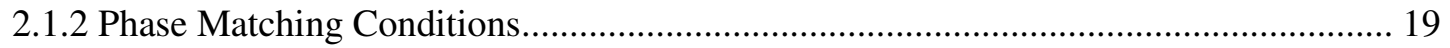

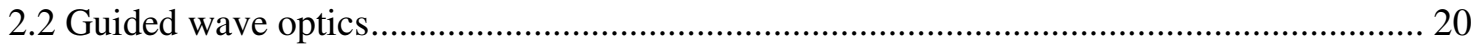

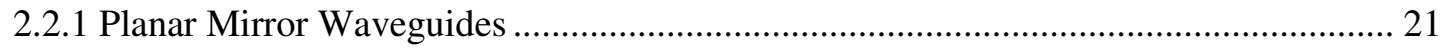

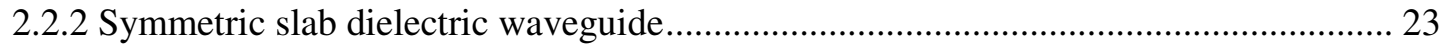

2.2.3 Analysis of Symmetric Slab Dielectric Waveguides .................................................. 24

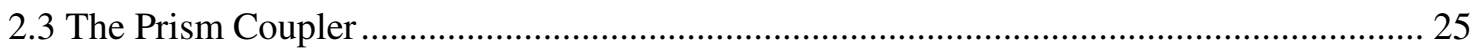

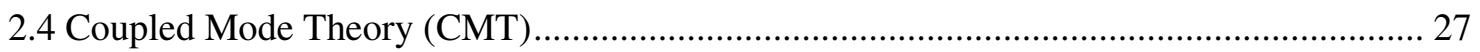

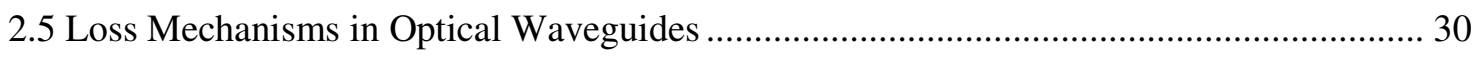

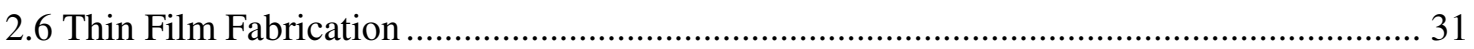

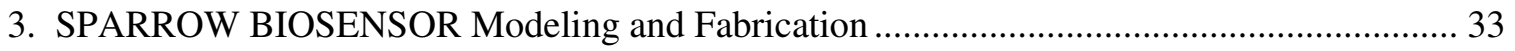




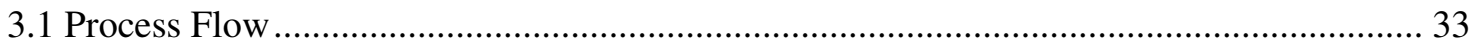

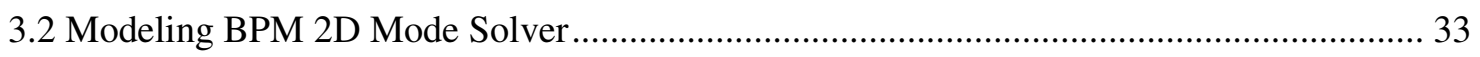

3.3 Waveguide Film Stack Growth using Electron-Beam Evaporator ..................................... 39

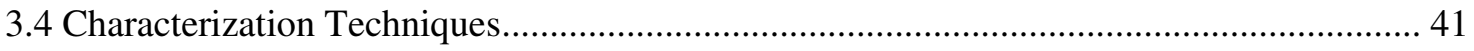

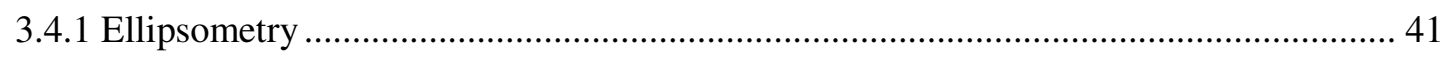

3.4.2 Prism Coupler and Effective Refractive Index [28] .................................................... 41

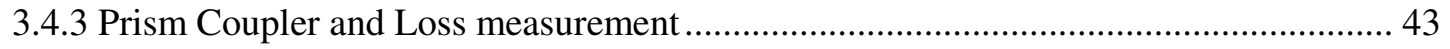

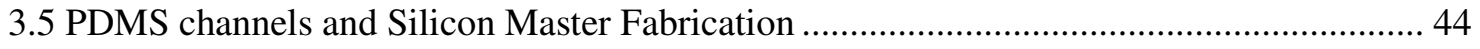

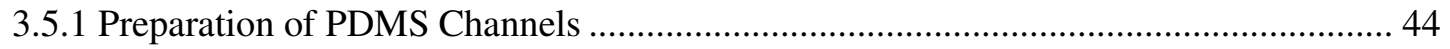

3.5.2 Process flow for Fabrication of Silicon Master.............................................................. 45

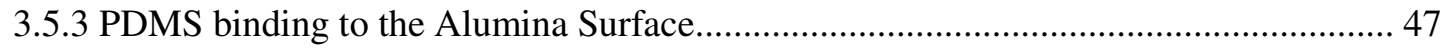

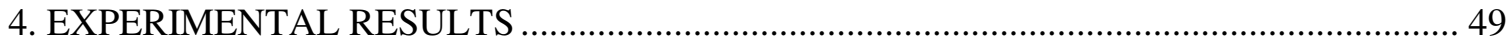

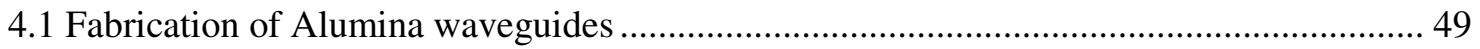

4.1.1 Refractive index function of Ion source parameters \& Optical Propagation Loss

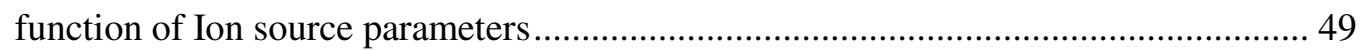

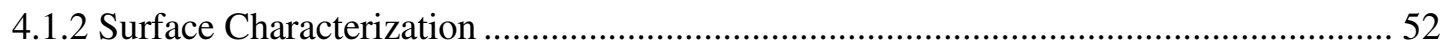

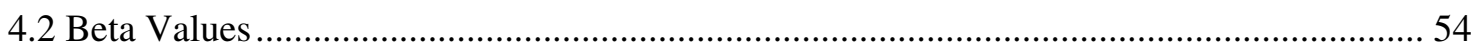

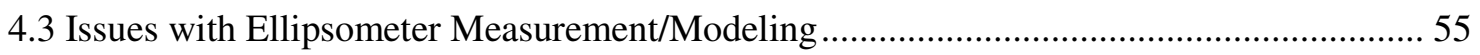

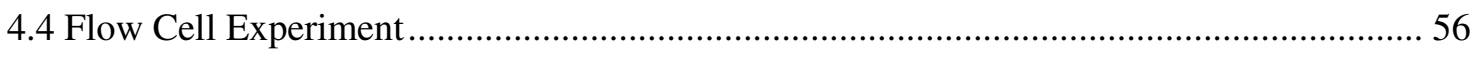

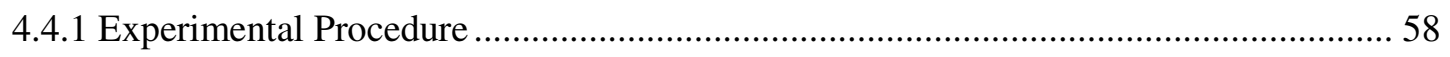

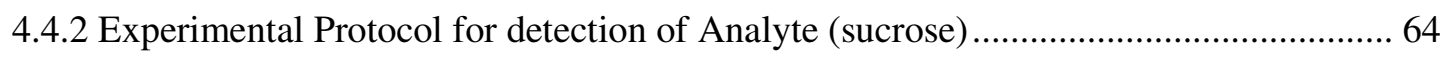

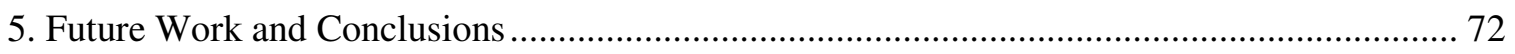

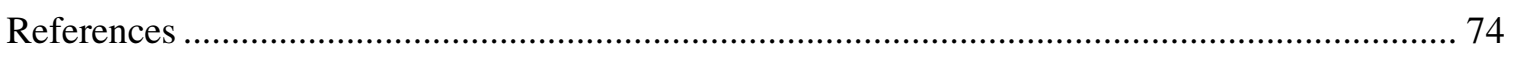

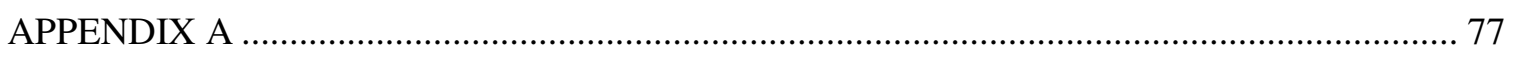

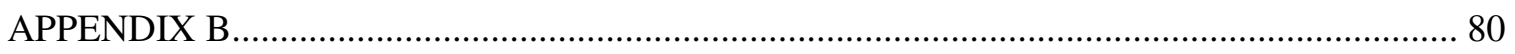

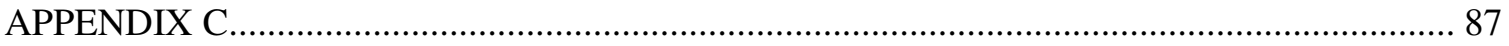




\section{List of Figures}

Figure 1.1 Classification of biosensors based on potential applications..................................... 2

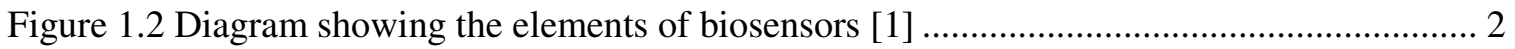

Figure 1.3 shows the bioelements and the sensing elements of biosensors [1] ........................... 3

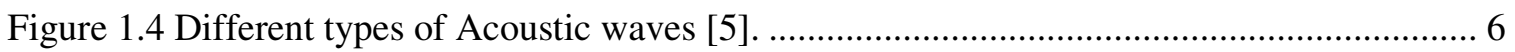

Figure 1.5 Schematic of an FPW device. The side view shows the different layers and the membrane movement. Interdigitated electrodes are used for actuation [7] .................. 7

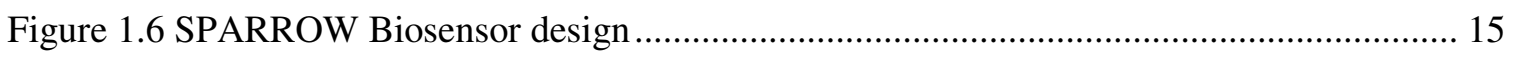

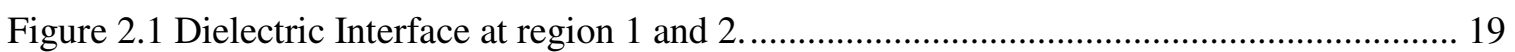

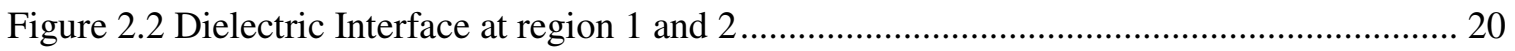

Figure 2.3 propagation of wave in a Planar Mirror waveguide ................................................. 21

Figure 2.4 shows the waves traveling along a symmetric slab dielectric waveguide.................... 23

Figure 2.5 Prism coupler coupling light into slab waveguide ................................................. 26

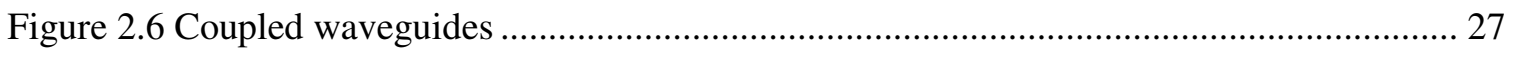

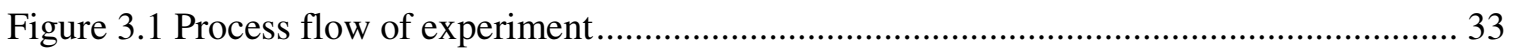

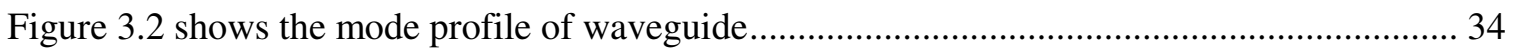

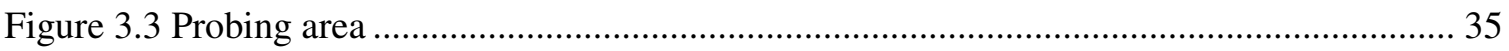

Figure 3.4a Simulation model for a sample structure................................................................ 37

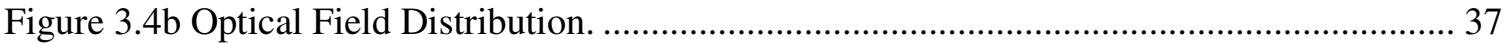

Figure 3.5 the model with the green color layers of Alumina for simulation in OPTIBPM 7.0 ... 37

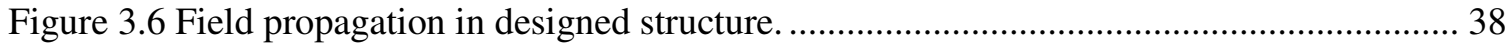

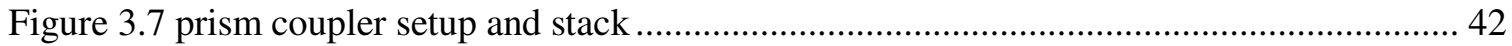

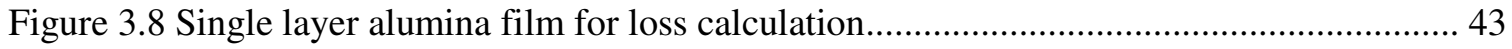

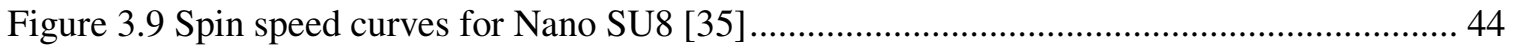

Figure 3.10 Process flow for silicon master fabrication and photomask .................................... 45

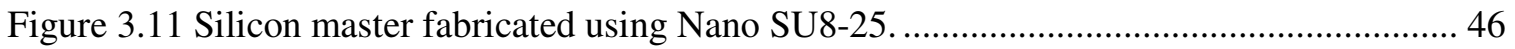

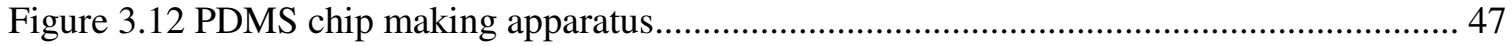

Figure 4.1 Variation of propagation loss for various oxygen flow rates with ion source drive

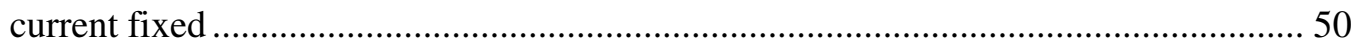

Figure 4.2 Optical propagation loss as a function of Ion source Drive current............................ 51

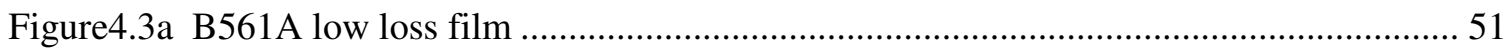

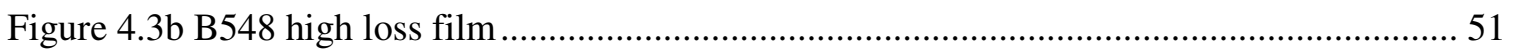

Figure 4.4 Refractive index as a function of Ion source Drive current ....................................... 52 
Figure 4.5 Surface characterization done on two different films with low loss and high loss film52

Figure 4.6 Optical propagation loss as a function of oxygen flow rate. 53

Figure 4.7 AFM image left (2000 nm x $2000 \mathrm{~nm}$ ) RMS roughness of $0.28 \mathrm{~nm}$, right (5000 nm x

$5000 \mathrm{~nm}$ ) RMS roughness of $0.48 \mathrm{~nm}$ of bare alumina surface. 53

Figure 4.8 SEM image of a single alumina layer (sample B540A) ............................................. 54

Figure 4.9 EDS for the above sample to check for contamination............................................... 54

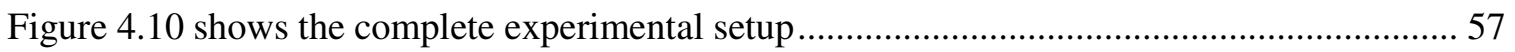

Figure 4.11a shows observation area with light coupled into waveguides................................... 58

Figure $4.11 \mathrm{~b}$ shows the experimental setup in the Laser lab (WVU).......................................... 58

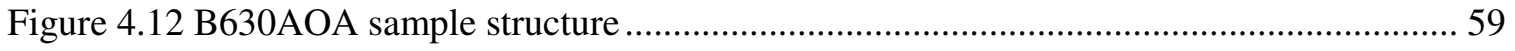

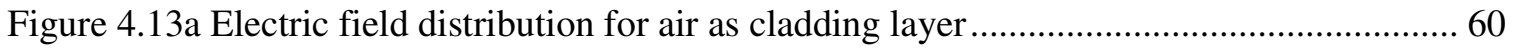

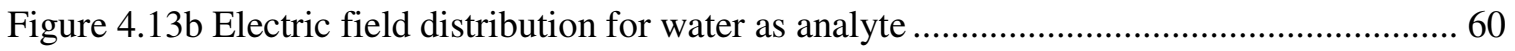

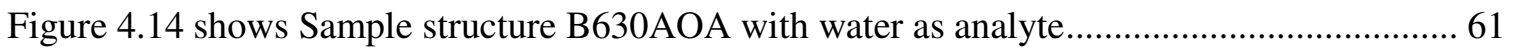

Figure 4.15 Loss variation in the observation area for different analyte solutions ...................... 62

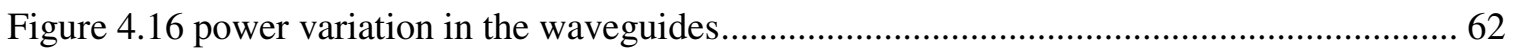

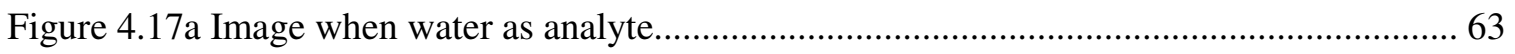

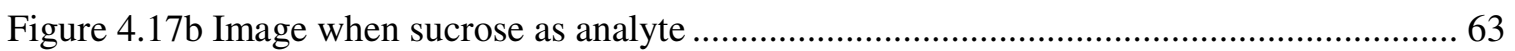

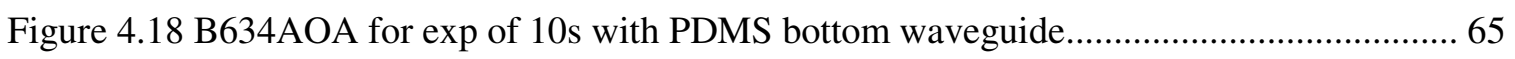

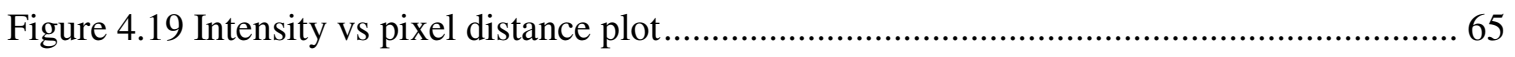

Figure 4.20 B634AOA for exp of 10s with PDMS bottom waveguide with SU 8 ....................... 66

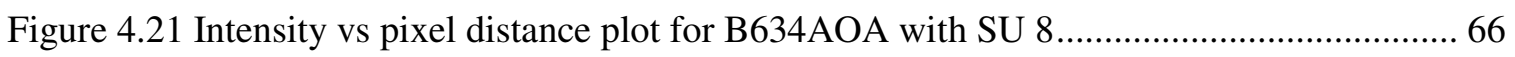

Figure 4.22 B634AOA for exp of 10s with PDMS bottom waveguide with water in the channel 66

Figure 4.23 B634AOA for exp of 10s with PDMS bottom waveguide with water rinse.............. 67

Figure 4.24 Intensity vs pixel distance plot for B634AOA for water and water rinse .................. 67

Figure 4.25 B634AOA for exp of 10s with PDMS SU8 2\% sucrose bottom waveguide ............. 68

Figure 4.26 B634AOA for exp of 10s with PDMS SU8 2\% sucrose + rinse (9.04) bottom

waveguide 68

Figure 4.27 Intensity vs pixel distance plot for B634AOA for PDMS SU8 2\% sucrose + rinse (9.04). 69

Figure 4.28 B634AOA for exp of 10s with PDMS SU8 2\% sucrose + rinse(9.04) + water....

Figure 4.29 The response for normalized output power vs Index of cladding (analyte) $1200 \mu \mathrm{m} 70$ Figure 4.30 The response for normalized output power vs Index of cladding (analyte) $1275 \mu \mathrm{m} 70$ 


\section{List of Tables}

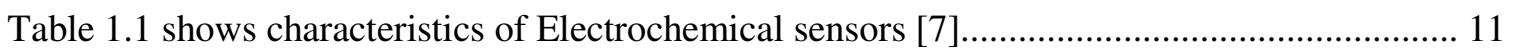

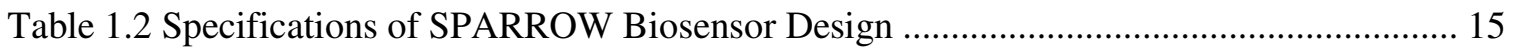

Table 2.1 shows the applications of thins films in various fields [31] ...................................... 31

Table 4.1 shows different sample grown under different drive currents with fixed oxygen flow rate

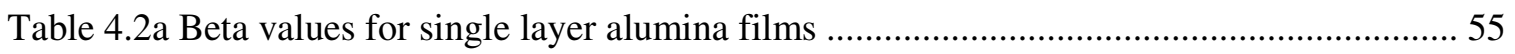

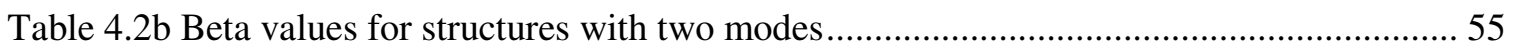

Table 4.3 shows the data of top and bottom waveguide in a stack.............................................. 56

Table 4.4 Data obtained from Simulations, Ellipsometer and Prism Coupler............................... 65

Table 4.5 Optical Propagation Loss Data obtained for each step............................................... 69 


\section{Introduction to Biosensors and Overview}

With the increased threat of using biological agents as weapons has emerged, there is great demand for a device that can rapidly, consistently, and accurately detect biological agents. Biosensors can serve as low cost and highly efficient devices for bioagent detection as well as in day-to-day applications such as medicine, agriculture, and biotechnology. A biosensor is a device that detects, records and transmits information associated with the recognition of a chemical, molecule or biological material or biomolecule in the environment. "A biosensor is an analytical device incorporating a deliberate and intimate combination of a specific biological element (that creates a recognition event) and a physical element (that transduces the recognition event)," as defined by S.P.J. Higson and D.M. Frazer [1].

\subsection{Theory of Biosensors}

\subsubsection{Biosensors Design and Applications}

The first biosensors were the enzyme electrodes developed by Leland C. Clark in the year 1962. Since then many biosensing devices were developed which are more refined, reliable, and mature. Biosensors are widely applied for the measurement both in vitro and in vivo. Figure 1 shows the various applications of biosensors. A major application is in blood glucose sensing because of its copious market potential. The range of biosensor applications has been extended from clinical laboratories to the point of care. Glucose meters for patients have been commercialized. Enzyme electrodes have been dedicated to critical care. Analyte combinations of electrolytes, blood gases and key metabolites are used for diagnostics under critical metabolic circumstances. The detection of nucleic acids, action of drugs on receptors, and transmitter-receptor interactions are some more applications of biosensors [2]. 


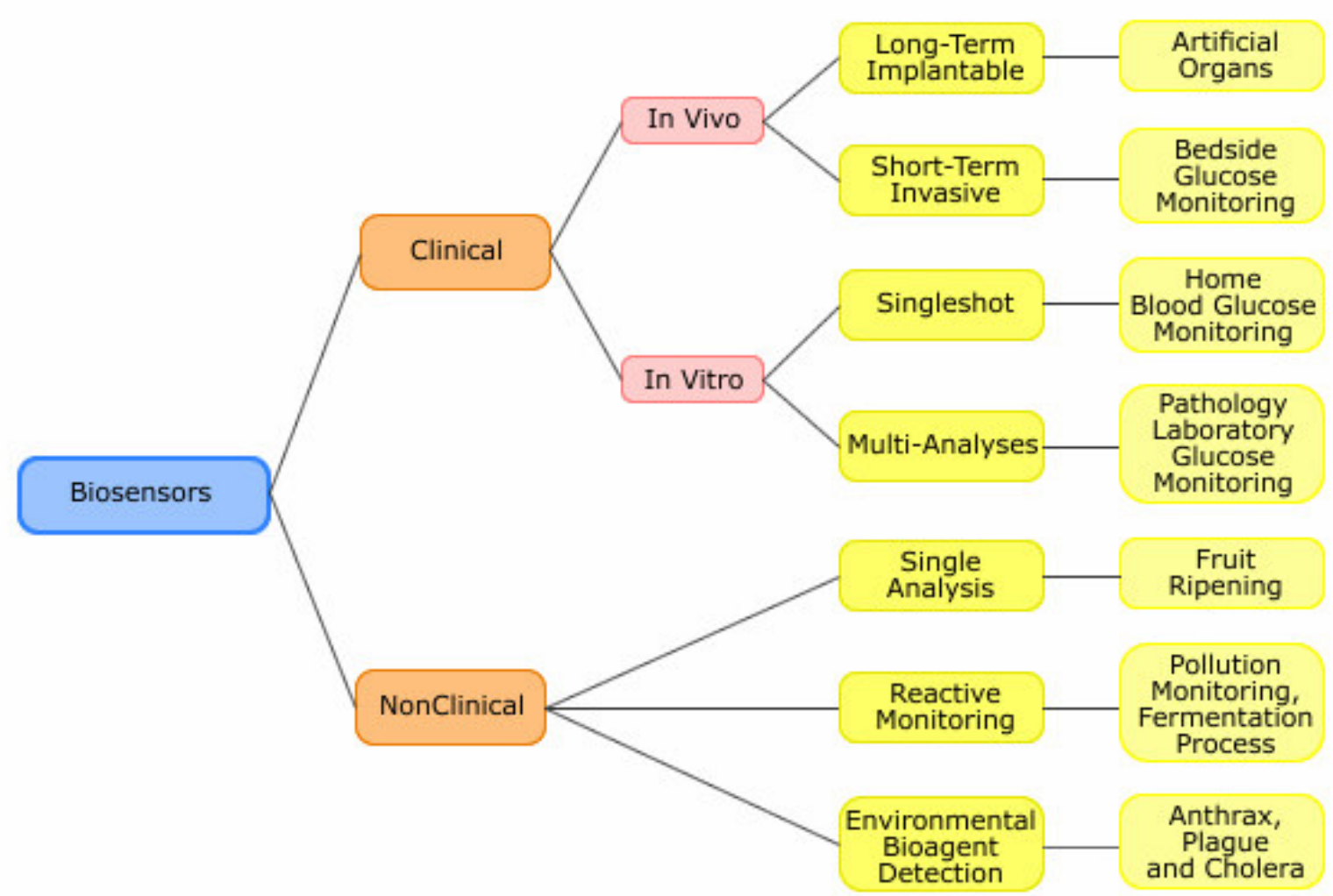

Figure 1.1 Classification of biosensors based on potential applications.

A biosensor consists of a bioelement and a sensor element, the schematic diagram of biosensor device is shown in Figure 1.2.

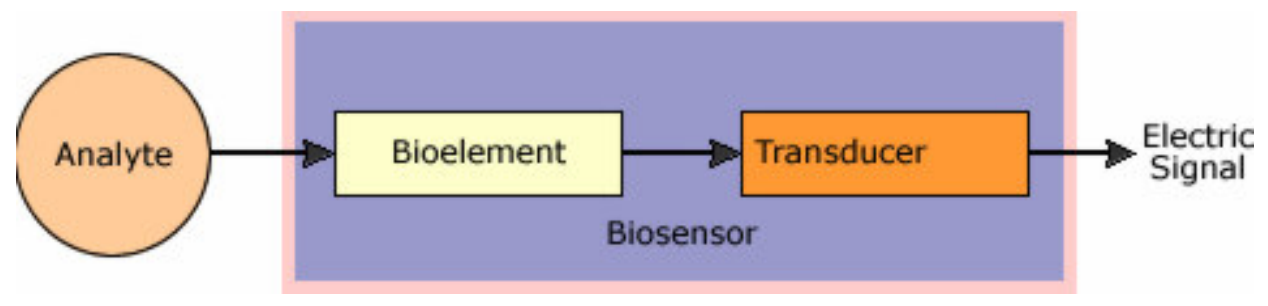

Figure 1.2 Diagram showing the elements of biosensors [1]

Depending on the analyte, a specific bioelement recognizes the analyte and the sensor element which is a transducer senses the change in the biomolecule and converts into an electrical signal. Ideally, the bioelement only recognizes a specific analyte and is insensitive to others. The bioelement can be an enzyme, antibody, living cells, or tissue. The sensing element can be electric current, electric potential, etc. 


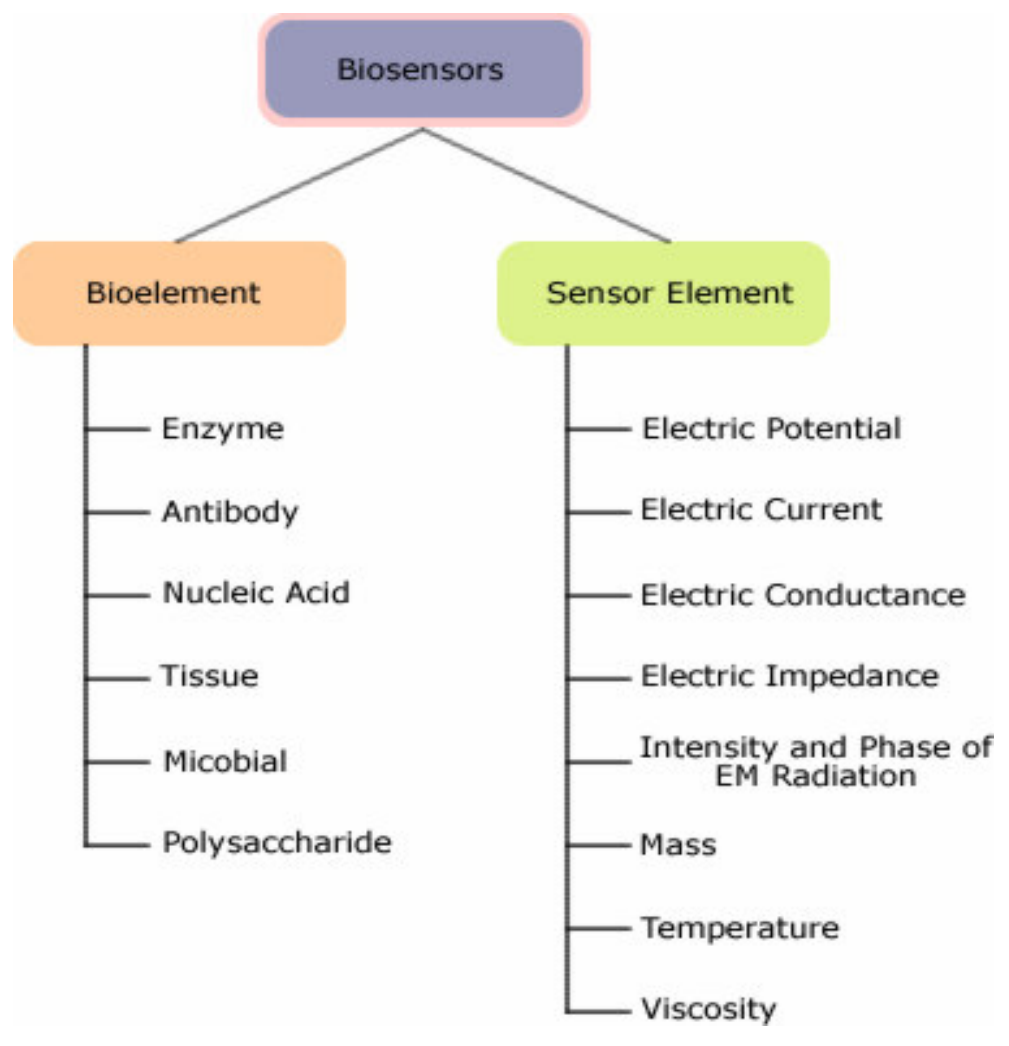

Figure 1.3 shows the bioelements and the sensing elements of biosensors [1].

Biosensors can produce signals modulated by the concentration of analytes either directly or indirectly. A direct action sensor measures a signal due to production of heat, light, chemical products whose strength is proportional to analyte concentration. There are four general ways that the biological target species and sensor elements can be coupled and they are Membrane entrapment in which a semi permeable membrane separates the analyte and bioelement and the sensor is attached to the bioelement. Physical adsorption which is a combination of van der Waals forces, hydrophobic forces, hydrogen bonds, and ionic forces to adhere the biomaterial to the surface of the sensor. Matrix entrapment, in this scheme a porous encapsulation matrix forms around the biological material that helps in adhering to it to the sensor. Lastly in the covalent bonding mechanism the sensor surface is treated as a reactive group to which the biological materials can bind.

\subsubsection{Common types of biosensors}

\section{Glucose biosensors}


The first experiment carried out by Leland C. Clark served as the origin of glucose biosensors [1]. Amperometric glucose biosensors are commercially used and are available in the market as glucose pens and glucose displays. Glucose sensors are prime examples of direct action biosensors [3]. Leland C. Clark used platinum (Pt) electrodes shown in to detect oxygen. The activity of the enzyme changes depending on the oxygen concentration in the surroundings, the enzyme glucose oxidase (GOD) was physically trapped against the electrodes with a piece of dialysis membrane. Glucose reacts with GOD to form gluconic acid by producing two electrons and two protons. In turn this reduced GOD reacts with the electrons and protons to form hydrogen peroxide and oxidized GOD, thus the higher the glucose the more oxygen is consumed [1]. These amperometric biosensors are disposable. Glucose biosensors are used for diabetic selfmonitoring of capillary blood glucose.

\section{Biosensor to monitor cell morphology}

This type of biosensor is used to monitor cell morphology in tissue culture environments, based on the principle known as electric cell-substrate impedance sensing. Impedance is measured across the electrodes due to changes caused when cells get attached and spread on the electrodes. This biosensor has many advantages such as it is less time consuming, possible to automate and quantify cell morphology measurements, and the signature for a cell can be assigned by using the fluctuating pattern.

\section{Mechanical Resonance}

Electrochemical mechanical oscillator can be used as a biosensor, similar to intracavity sensing. Changes in effective mass result in changes the resonance frequency. Electrochemical sensors are implemented with quartz crystal.

\section{Fiber-optic sensors}

In the field of communication and semiconductor industries rapid innovations have been made to produce low-loss fibers, circuitry for processing optical signals, integrated beam splitters and wavelength filters and spectrally pure light sources such as LEDs (light-emitting diodes) and semiconductor lasers [4]. At the National Institutes of Health, Gerald G. Vurek and Robert Bowman in 1969 exhibited the first fiber-optic 
sensor which is a calorimeter that measured the binding of dyes to kidney tubules. Fiberoptic sensors are classified into three categories. The first is a straightforward extension of electronic biosensor techniques detecting changes in a target substance's optical properties rather than the electrical properties. This type of sensor consists of a cell bounded by a semi permeable membrane, reagents inside the membrane, a fiber to illuminate the cell and detectors to measure the change in optical properties.

The other two categories are based on evanescent-wave and surface plasmon devices which depend upon the way the optical fibers transmit light. The evanescent wave biosensor senses competitive binding directly, these detectors are based on the fact that in an optical fiber the optical energy is not only confined to the core and travels within the core but travels through a region that extends outward on the order of a few hundreds of nanometers into the medium surrounding the core.

A surface plasmon device is made of thin metal film deposited on the surface of a thin piece of glass that acts as a light guide. The plasmon or the pathway provided by the conductive film changes the critical angle of incidence at which light is trapped within the glass, this changed critical angle depends sensitively on the amount of material adsorbed on the metal film. As this method does not require labeled molecules and competitive assays, it can be used for a wide range of substances.

In resonant wave biosensors an acoustic wave transducer is coupled with an antibody or bioelement. The change in the mass of the membrane when an analyte molecule or antigen adheres to the membrane results in a subsequent change in the resonant frequency of the transducer. Biosensors made based on the acoustic wave technology are sensitive, accurate, small, portable, robust and have excellent aging properties [5]. Acoustic waves can be generated by a variety of means such as piezoelectric, magnetostrictive, optical and thermal. An acoustic sensor has frequency as the output sensor signal, which is a function of the magnitude of the parameter being measured. 


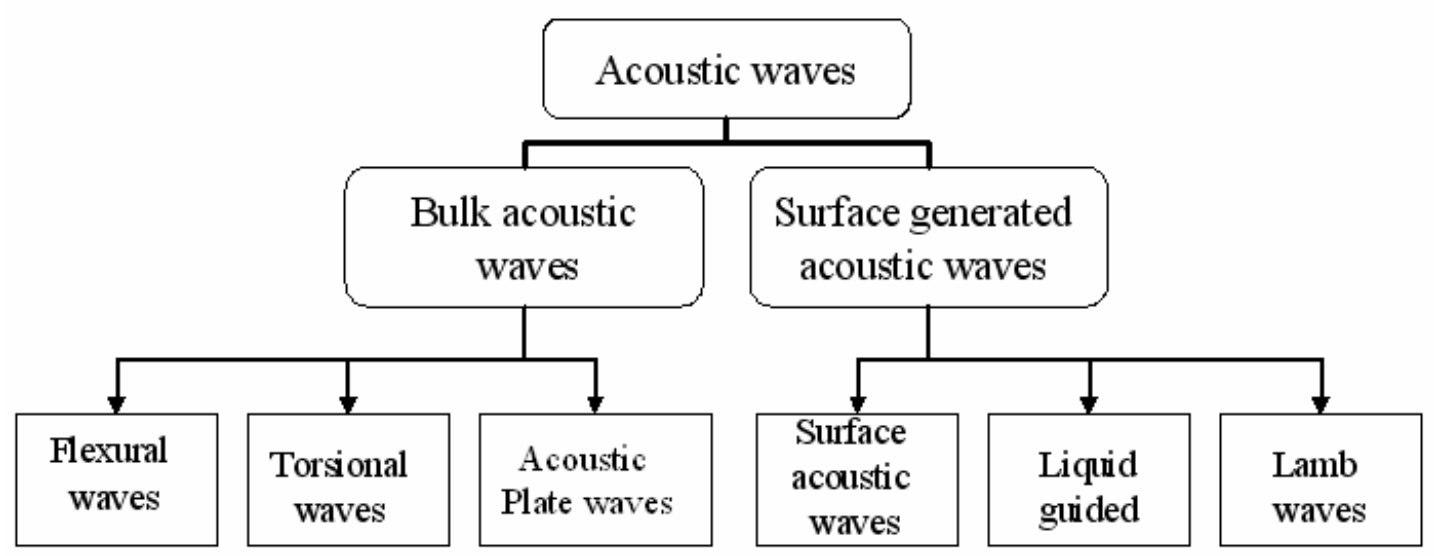

Figure 1.4 Different types of Acoustic waves [5].

Progress in biosensors has been enormously influenced by the sensor technology. The direct signal transfer of the signal gained in the analyte recognition from the biocomponent to the transducer offer distinct advantages as compared with a process exploiting the mass transfer of one reaction partner:

First, the loss of sensitivity by migration of the signal mediating component into the bulk phase can be minimized.

Second, the direct transfer is restricted to the phase boundary at the transducer, thus restricting contributions of the bulk phase.

Optical techniques such as surface plasmon, resonance, reflectometric interference spectroscopy, and grating couplers do not require a signal generating reaction partner [2].

\subsection{Classification Based on Transduction Principle}

Based on the transduction principles used [6] biosensors can be classified as

1. Chemomechanical or mass-sensitive sensors

2. Thermal sensors

3. Electrochemical sensors

4. Optical sensors 


\subsubsection{Chemomechanical or Mass-Sesnsitive sensors}

Chemomechanical or Mass-Sensitive sensors are based on the principle of mass changes upon absorption. The species to be sensed has to be immobilized for the sensor to respond. Mass changes are monitored in two ways. One is by deflecting a micromechanical structure due to stress changes or static measurements i.e., mass loading and the second is by determining frequency change in proportion to stress changes or mass loading on the device [7]. Some of the chemomechanical devices are TSMR (thickness shear mode resonator), QMB (quartz microbalance), and the SAW (Rayleigh surface acoustic wave) devices. These devices are based on piezoelectric quartz substrates. Flexural-plate-wave or lamb-wave devices (FPWs) and micro machined cantilevers are based on semiconductor technology.
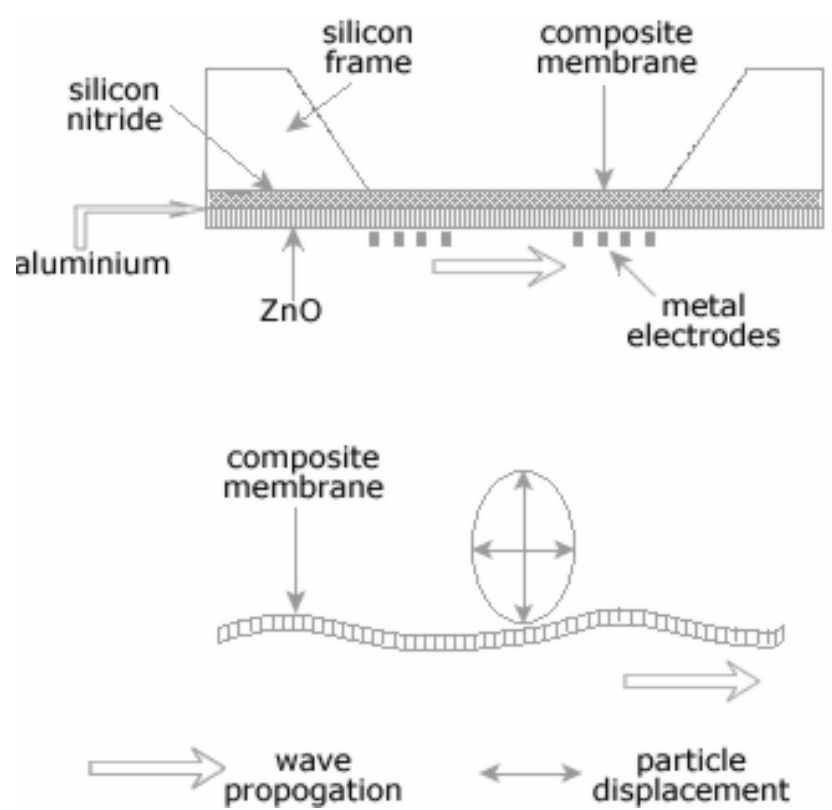

Figure 1.5 Schematic of an FPW device. The side view shows the different layers and the membrane movement. Interdigitated electrodes are used for actuation [7].

1) Flexural-Plate-Wave or Lamb-Wave Devices: These devices have high sensitivity and mass at a low operating frequency. On these devices there are interdigital transducers that generate flexural waves having retrograde elliptical particle motions as in SAW devices Figure 1.5. A series of plate modes are generated by these flexural waves and one of these has a lower frequency than the other possible modes. With decreasing plate thickness the velocity of this unique wave decreases and the plate is set into motion 
[8]. High mass sensitivity results from the confinement of the acoustic energy. There is flexibility with this device as the sensitive layer can be deposited on either side of the membrane. The fabrication steps involved are first to deposit $\mathrm{Al}$ (sputtering) and $\mathrm{Si}$ nitride (LPCVD), back-side etching $(\mathrm{KOH})$ to obtain the membrane structure, zinc oxide deposition, for IDT processing aluminum or gold are either evaporated or sputter coated then the polymers are spin or spray coated and finally biological particles are deposited. FPWs are used in the detection of different organic volatiles in the gas phase by polymeric layers and in the detection of breast cancer antigens [9], [10], [11].

2) Resonating Cantilevers: A mass-sensitive cantilever is a layered structure having, silicon, silicon oxide/nitride, and metallization. The base of the cantilever is fixed to a silicon chip and the free suspended end of the cantilever is coated with a sensitive layer. The cantilever can be operated in two modes. 1) Static mode: the stress changes or mass loading causing cantilever deflections are measured by means of a laser via beam reflections. The cantilever has to be large and flexible to get large deflections, and can be used in liquid media. 2) Dynamic mode: when there is mass loading the change in the resonant frequency upon excitation of the cantilever in its fundamental mode is measured in correspondence to other mass-sensitive transducers [7]. The setup for this method is simple as it requires short and stiff cantilevers to obtain higher operating frequencies. Piezoelectric materials are used for excitation of cantilevers and piezoresistors embedded in the cantilever base detect the cantilever deflection [12]. Fabrication steps involved [13] are first bulk micromachining by backside etching $(\mathrm{KOH})$ to obtain a membrane structure, then RIE on the wafer front side for release of the cantilevers. Finally layers are coated and biological entities are deposited. Cantilever sensors find applications in biosensing in liquids such as hybridizations and in the detection of complementary strands of DNA fragments (oligonucleotides) [11].

\subsubsection{Thermal Sensors}

Thermal Sensors are also known as calorimetric sensors. Thermal detection biosensors detect the changes in the temperature when a reaction takes place in a medium, this change is temperature is due to either absorption or production of heat when reactions occur. Thermal biosensors do not need recalibration as other transducers and do 
not respond to optical and electrochemical properties of the sample. These sensors are a combination of immobilized enzyme molecules with temperature sensors and are based on the principle of measuring the enthalpy change produced by the chemical to be detected this change in temperature is converted into an electrical signal by the transducer. Chemical reactions are of two types: exothermic reactions which release heat and endothermic reactions which absorb heat from the surroundings. At thermodynamic equilibrium there is no heat produced hence no measurable signal, therefore many competing restrictions are applied while designing thermal sensors such as the desire to thermally isolate the sensing area for improved the sensitivity the sensing area while at the same time interacting with the chemical species [7].

1) Catalytic Thermal Sensors also called Pellistors use a resistance thermometer placed near the catalyst measure the heat generated during the combustion of flammable gaseous compounds in ambient air on the surface of the hot catalyst. This device consists of a ceramic bead having a platinum coil. The ceramic bead is used as the sensor signal is directly proportional to the active surface area. Catalytic thermal sensors are used in monitoring and detection of flammable gas hazards in industrial, commercial and domestic environments.

2) Thermoelectric or Seeback-Effect-Based Sensors: If two different semiconductors are joined together at the hot point and a temperature difference $\mathrm{T}$ is maintained between this point and the cold point, then an open circuit voltage $\mathrm{V}$ is developed between the two different leads at the cold point. This voltage is proportional to the difference of Fermi levels of the two materials and proportional to the temperature difference. This effect, called the Seebeck effect after its discoverer T. J. Seebeck (1770 - 1831) [6]. Thermocouples are connected in series to form a thermopile to obtain higher thermoelectric voltage. Applications include the detection of different kinds of organic volatiles in the gas phase by using polymeric layers and the biosensing of glucose, urea, and penicillin in the liquid phase.

\subsubsection{Electrochemical sensors}

Electrochemical sensors make use of electrochemical or charge-transfer reactions from an electrode to a solid or liquid phase or from liquid to solid phase. Chemical 
changes take place at the electrodes or in the probed sample volume, and the current is measured across them. The charge transport in the sample can be ionic or electronic or both and the transducer branch is electronic [7]. These sensors are far advanced and are commercially used. Electrochemical biosensors are mainly used for the detection of DNA, DNA-binding drugs, and glucose concentration. The principle of the biosensors is to sense the change in the electrical properties of the solution undergoing chemical reactions and producing or consuming ions or electrons and the changes in the electrical properties are measured. Classification of the electrochemical sensors is based on their electro-analytical principles [14]. Based on the current-voltage relationship measurement the sensors are called voltammetric sensors. Chemoresistors based on conductometry measure conductance by applying an ac potential with small amplitude to a pair of electrodes in order to prevent polarization. When an analyte interacts with the film the changes in the electric conductivity are measured. These chemoresistors are classified as high temperature and low temperature chemoresistors. The high temperature chemoresistor requires a thermally isolated stage like a suspended membrane allowing the sensing materials to maintain high temperature, an integrated heater, electrodes and a temperature sensor [15]. Applications include the detection of inorganic gases such as hydrogen, oxygen, nitrogen oxide, carbon monoxide and organic volatiles.

Potentiometric sensors measure the potential at an electrode at equilibrium state without any current flow and this potential is proportional to the logarithm of the concentration of the electro-active species. Chemotransistors are based on the FET (fieldeffect transistor). When a positive voltage is applied to the gate electrode of a n-channel MOSFET [13] (metal- oxide-semiconductor field-effect transistor), a conducting channel is created between source and drain and the conductivity of the channel can be varied by changing the strength of the electrical field between gate electrode and the silicon perpendicular to the silicon surface [16]. The sensitivity and selectivity patterns of gassensitive FET devices depend on the type and thickness of the catalytic metal. Applications of FET sensors include detection of ammonia, amines and molecules that give rise to polarization in a thin metal film or cause dipoles on the insulator surface [7].

The conductometric sensors have relatively low sensitivity compared to amperometric sensors. 


\begin{tabular}{|c|c|c|c|}
\hline & Conductometric sensors & Amperometric sensors & Potentiometric sensors \\
\hline Measured Parameter & Conductance/Resistance & Current & PotentialVoltage \\
Applied Voltage & Sinusoidal (AC) & Constant Potential (DC) & Ramp Voltage \\
Sensitivity & Low & High & \\
Governing Equation & Incremental Resistance & Cottrell Equation & Nerst Equation \\
Fabrication & FET + Enzyme & FET + Enzyme & FET + Enzyme \\
& & 2 Electrodes & Oxide electrode \\
\hline
\end{tabular}

Table 1.1 shows characteristics of Electrochemical sensors [7].

\subsubsection{Optical Sensors}

Optical techniques are chosen as they offer good selectivity and various transduction mechanisms when compared to other sensing methods. The output signal measured from the transducer is light. This technique uses characteristic properties of the electromagnetic waves like amplitude, frequency, state of polarization, phase, and scattering [17]. The biosensor can be made based on electrochemiluminescence, surface plasmon, resonance, reflectometric interference spectroscopy, and grating couplers. The wavelength of the radiation can be tuned to match the energy of desired resonance or absorption process. When a sample is illuminated with electromagnetic waves the following effects can occur, the radiation can be reflected (metallic reflection, total internal reflection involving evanescent waves), refracted, scattered, absorbed, at the interface or can produce phosphorescence/fluorescence (absorption-emission process) or chemiluminescence (conversion of chemical energy into light) [18]. The following are the semiconductor and microoptoelectromechanical based transducers.

1) Integrated Mach-Zehnder Interferometer: Planar waveguides with guided waves or modes are used to make integrated optical (IO) sensors. High refractivity silicon dioxide or titanium dioxide and silicon nitride films are used as waveguides on oxidized silicon substrates. Transverse electric (TE) or s polarized, surface-normal and transverse magnetic (TM) or p polarized, surface-parallel are the modes or guided waves in planar optical waveguides. Whenever there are changes in the refractive index distribution in the proximity of the waveguide surface within the penetration depth of few hundred nanometers of the evanescent field in the sample then the there are changes in the effective refractive index of a guided mode. The evanescent field dies out exponentially 
with increasing distance within the waveguide surface area. Evanescent waves are formed when sinusoidal waves are internally reflected off an interface at an angle greater than the critical angle so that total internal reflection occurs. Changes in the effective refractive index can be induced by absorption of an adlayer onto the surface of the waveguide from gas or liquid phase or by interaction of an analyte molecule on the waveguide surface [15]. The change in the effective refractive index of the waveguide is smaller than the change in the refractive index of the cladding. Phase changes can be detected as a consequence of changes in propagation constant

$$
\beta=n_{e} k_{o}
$$

where

$k_{o}=\frac{2 \pi}{\lambda}$ and $n_{e}:$ the effective refractive index.

The change in the effective refractive index is of the order of 0.1 times the change in the refractive index of the cladding $n_{c}$ for the classic dielectric waveguide [19]. In this device the relative phase change observed in is given by

$$
\frac{\Delta \varphi}{2 \pi}=\frac{\Delta n e_{f f} l}{\lambda}
$$

Equation $2, l$ is the interaction length and the sensitivity is proportional to the interaction length.

Mach-Zehnder Integrated Optical $[\mathrm{IO}]$ devices are single mode channel waveguides. A waveguide divided into an open measurement path and a protected reference path and recombined after some distance as shown in Fig. In the sensing path the phase difference is detected by interference effects introduced by the refractive index change due to analyte interaction.

The fabrication steps for Mach-Zehnder IO devices are patterning of silicon nitride as waveguide (LPCVD, RIE, lithography) [20], deposition of an silicon oxide cladding layer (PECVD) [20], and deposition of the chemically sensitive layer or immobilization of biological entities [19].

Mach-Zehnder IO devices are used to detect different organic solvents in liquid phase, and biotin/streptavidin-mediated immunosensing involving antibody-antigen binding [20]. 
2) Fabry-Pérot-Based Microspectrometer (FPI): This device consists of two partially reflecting, low-loss, and parallel mirrors separated by a gap [17]. If this gap is equal to multiples of half a wavelength of the incident light then a series of sharp resonant transmission peaks due to multiple reflections of the light in the cavity occurring through the mirrors. Small changes in the gap can produce large changes in the transmission response and this can be achieved by using highly reflecting mirrors also the transmission is not only the function of gap spacing but also the radiation wavelength. Fabrication steps include wafer fusion bonding then etching is done to achieve movable mesa, again etching is performed to achieve the gap and control electrode spacing, and finally to multilayer dielectric mirror layers and control electrodes have to be deposited [17]. Applications include gas sensors to monitor carbon monoxide and hydrocarbons.

3) Surface Plasmon sensors are the most commercially used sensors but are expensive and have the problem of portability and are not applicable to the real time applications due their large size. Photonic crystal sensors draw upon changes in transmission properties in the crystal upon similar changes in material optical characteristics arising from binding within selected regions of the crystal lattice [21]. Both evanescent (explained in next section) and photonic crystal based sensors are important as they hold significant potential for integration and are the subject of intense investigation. In our project work here we concentrate on evanescent wave technique to design our model of SPARROW biosensor.

\subsection{Evanescent Wave}

Evanescent wave based sensors are based on the interaction of evanescent wave with the bioagent and the resultant change in the wave parameter is used to detect the presence of the bioagent. The sensitivity and the portability are the primary characteristics of evanescent wave-based optical biosensor. Since they are surface sensitive these sensors are used to detect organic bioagents which are grown on the surface. The surface detects the bioagent by determining the change in its optical characteristics. 
An evanescent wave is an electromagnetic wave propagating along the direction of light decay exponentially with distance. Evanescent waves are formed when sinusoidal waves are internally reflected off an interface at an angle greater than the critical angle so that the internal reflection occurs. Their existence is due the reason that the electric and magnetic fields cannot be discontinuous at a boundary. "Evanescent" means tends to vanish, as the intensity of evanescent waves decays exponentially with the distance from the interface at which they are formed. When light travels from a medium of higher refractive index to a medium of lower refractive index and exceed the critical angle give rise to evanescent waves.

$$
E=E_{o} \exp \left(-x / d_{p}\right)
$$

where ' $\mathrm{x}$ ' is the distance equal to which the intensity of the wave reduces to $1 / \mathrm{e}$ of the original intensity and ' $d_{p}$ ' is called the penetration depth [22].

\subsection{SPARROW BIOSENSOR Architecture}

\subsubsection{Fundamental Design of SPARROW Biosensor}

A current direction in the field of optical sensors is development of low cost compact sensors that can sense exactly the target that needs to be measured. The Stacked Planar Affinity Regulated Resonant Optical Waveguide (SPARROW) biosensor is a device being developed that exploits evanescent wave interactions and power transfer between two waveguides for biohazard detection and identification which is both compact and holds the potential for low cost device. The SPARROW sensor consists of two planar, single mode resonant coupled waveguides separated vertically by a lower refractive index silicon dioxide layer. In this study alumina waveguides were chosen for their stable physical (thickness and surface roughness) and optical (refractive index and loss) properties [23]. Aluminum oxide films can be produced by many techniques such as vacuum evaporation of $\mathrm{Al}_{2} \mathrm{O}_{3}$ [24]. It is desirable to have a material that exhibits low optical loss, is chemically and mechanically stable, and can be deposited easily with uniform thickness and also have a high contrast between the indices of the layers. For fabrication of such alumina optical waveguides, controlled highly precise thin film processes are required [23], we have employed a Temescal BJD-2000 electron beam deposition system with a Denton Vacuum CC-105 cold cathode gridless ion source. 
Borofloat was used as a substrate in order to evaluate the wave guiding properties of the deposited alumina film.

\begin{tabular}{|c|c|c|c|}
\hline & Materials & Thickness $(\mathrm{nm})$ & Refractive Index \\
\hline Top waveguide & Alumina $\left(\mathrm{Al}_{2} \mathrm{O}_{3}\right)$ & 194 & 1.659 \\
\hline Spacer & Silica $\left(\mathrm{SiO}_{2}\right)$ & 1100 & 1.457 \\
\hline Bottom waveguide & Alumina $\left(\mathrm{Al}_{2} \mathrm{O}_{3}\right)$ & 144 & 1.659 \\
\hline Substrate & Borofloat & 1000 & 1.4701 \\
\hline
\end{tabular}

Table 1.2 Specifications of SPARROW Biosensor Design

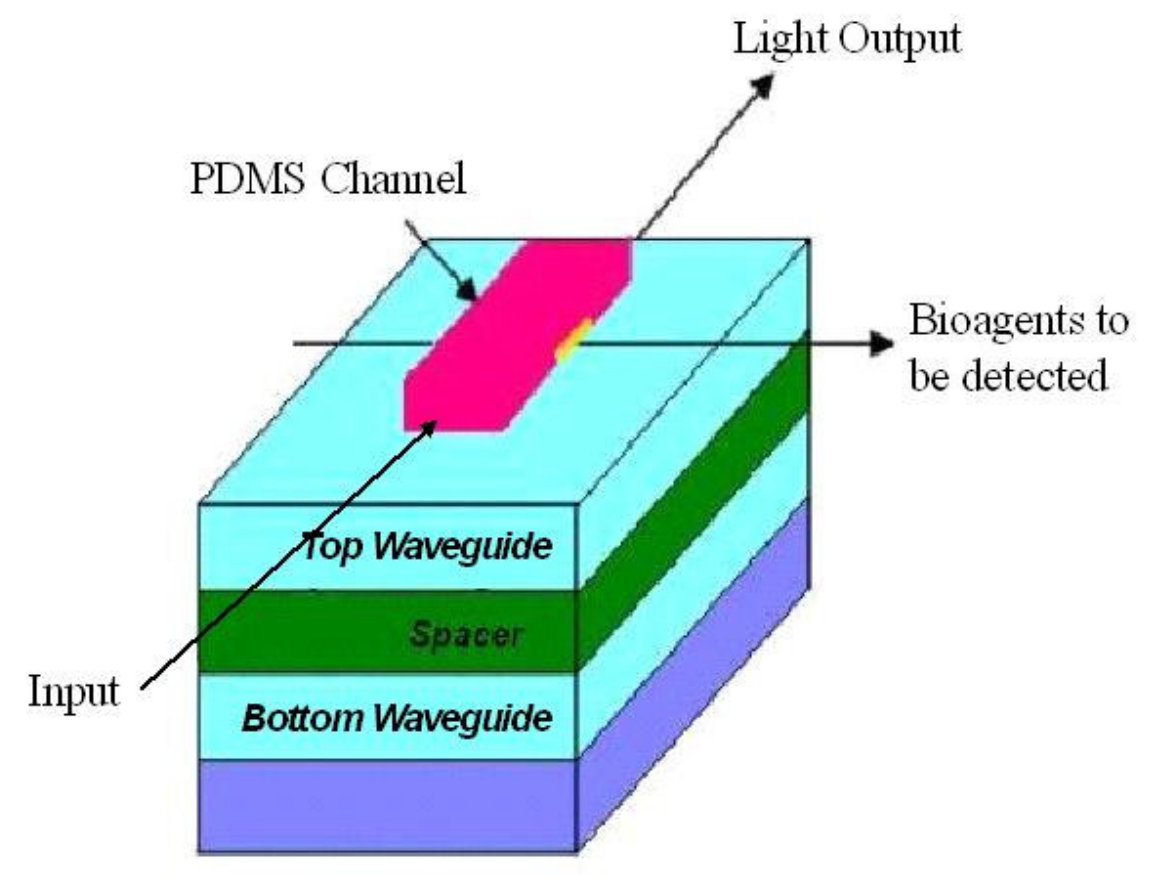

Figure 1.6 SPARROW Biosensor design

\subsubsection{Principle of Operation of SPARROW Biosensor}

A polarized coherent beam of light from Helium-Neon laser is introduced into the top waveguide. When the waveguides are under tuned condition, they optically resonate, and periodically exchange all of the input optical power, back and forth, along a fixed interaction length in the direction of light propagation and out to terminal. For sensing purposes, the top guide is covered with a thin surface layer of analyte. It has a biolayer which acts as a receptor, a stacked coupled waveguides (Figure 1.6) that acts as a transducer; a detector and CCD camera are used to register images to detect the power in 
the upper or lower guide after interaction with the biolayer. SPARROW biosensors work on the principle of resonance between the waveguides. The structure is comprised of two single mode planar waveguides of higher index material separated by spacer layer of lower index on a low index substrate. Optical power enters the top waveguide and under tuned conditions all the optical power is transferred in the lower waveguide from the upper guide after traversing some distance along the length of the device. The periodic exchanges of optical power take place between the two waveguides. The two waveguides act as tuned resonators and hence the name of resonant optical waveguide biosensor. This transfer of power continues over the whole length of the device. This condition is called phase match or a tuned condition. Properly designed, this tuning is strongly dependent on the effective refractive index of the medium above the top waveguide and any change in the effective index changes the strength of coupling between the two guides. The detection of a bioagent takes place at the top of the waveguide where any change in environment creates a change in the effective index. This changes the phase matched condition and detunes the two waveguides. This is the basis of transduction which detects any changes in the immediate environment at the surface of the upper waveguide.

In ideal operation of the device, there should be no power detected from the top waveguide when there are no bio-agents bonded on the biolayer. Presence of bioagents alters the effective refractive index of the biolayer and thus produces a change in the power detected at the ends of the waveguide. The change in power is correlated to the refractive index and thus the index of the bio-agent is determined.

The key issues of this sensor are designing and fabrication of coupled waveguides, bio-layer design, wave guide characterization and flow cell experiments. Fabrication of coupled waveguides as per the design is very important as a single nanometer change from the design would produce a huge variation in the power at the ends of the waveguide. The biolayer designed should be less in height as the sensitivity of the biosensor is within the penetration depth of the evanescent wave. This is usually around $100 \mathrm{~nm}$ and hence the biolayer height should be as less as possible. Power observed at the ends of the waveguide is dependent on attenuation present in the wave guide. Hence the waveguides have to be optically characterized to determine the actual power observed at the ends of the waveguide. The final issue is with the flow cell 
experiments. The employed setup should be such that there is no change in the coupling spot before and after the bio-agent bonding. This means that the whole setup meant for flow cell experiments has to be physically at the same location before and after the bioagent bonding.

This thesis aims at designing resonant coupled waveguides, performing waveguide characterization and setting up for flow cell experiments. Sensitivity analysis was also made to see the variation produced in power and coupling length due to change in thickness and refractive index of the top wave guide.

The goal of the thesis is to design, fabricate and to characterize SPARROW biosensor.

\subsection{Organization of the Thesis}

My work is organized as follows. Chapter 2 introduces the basic electromagnetic theory. This includes applications of ray optics and wave optics in slab waveguides, evanescent wave technique and coupling mode theory. In Chapter 3, complete process flow, modeling of waveguides using mode solver software and design of waveguides using OPTIWAVE software, fabrication method of stacked waveguide, characterization techniques and the PDMS channel and its binding to the top alumina surface. In Chapter 4, experimental results with the beta values, waveguide loss variation with ion beam parameters like oxygen flow rate and ion drive current, flow cell experiment and measurements. Based on this model, the SPARROW biosensor behavior can be predicted and optimized. In Chapter 5, is thesis summary and conclusion. 


\section{Theoretical Background}

This chapter introduces the basic electromagnetic theory and the concepts of light wave propagation and its behavior in waveguides. Waveguide attenuation is also detailed. The proposed design has two resonant coupled waveguides, so it is necessary to understand the principle of coupled mode theory and prism coupling technique.

\subsection{Basics of Electromagnetic Theory and Dielectric waveguides}

\section{Maxwell's Equations for a wave propagating in isotropic medium:}

Maxwell's equations give relationships between the electric and magnetic fields and are useful to analyze the optical waveguides. Assuming a linear, isotropic dielectric material having no currents and free charges, the equations take the form ${ }^{1}$

$$
\begin{array}{ll}
\nabla \times \vec{E}=-\frac{\partial B}{\partial t} & 2.1 \mathrm{a} \\
\nabla \times \vec{H}=\frac{\partial D}{\partial t} & 2.1 \mathrm{~b} \\
\nabla \cdot \vec{D}=0 & 2.1 \mathrm{c} \\
\nabla \cdot \vec{B}=0 & 2.1 \mathrm{~d}
\end{array}
$$

where $D=\varepsilon E$ and $B=\mu H$. The parameter $\varepsilon$ is the permittivity or dielectric constant and $\mu$ is permeability of the medium.

A relationship defining the wave phenomena of the electromagnetic fields can be derived from Maxwell's equations. Taking the curl of Eq. 2.1a and making use of Eq. $2.1 \mathrm{~b}$ gives

$$
\nabla \times(\nabla \times E)=-\mu \frac{\partial}{\partial t}(\nabla \times H)=-\varepsilon \mu \frac{\partial^{2} E}{\partial t^{2}} \quad 2.2 \mathrm{a}
$$

Using the vector identity

$$
\nabla \times(\nabla \times E)=\nabla(\nabla \cdot E)-\nabla^{2} E
$$

Using Eq.1c, Eq. 2a becomes

$$
\nabla^{2} E=\varepsilon \mu \frac{\partial^{2} E}{\partial t^{2}}
$$

Similarly, by taking the curl of Equation 1b, it can be shown that 


$$
\nabla^{2} H=\varepsilon \mu \frac{\partial^{2} H}{\partial t^{2}}
$$

Equations $2 \mathrm{~b}$ and $2 \mathrm{c}$ are the standard wave equations.

\subsubsection{Boundary Conditions at Dielectric Interfaces}

Electromagnetic wave behavior at the interfaces can be well understood from the boundary conditions. From Maxwell's equations [20]

$$
\begin{aligned}
& \oint_{c} E \cdot \hat{l} d l=-\frac{\partial}{\partial t} \oiint_{s} B \cdot \hat{n} d S \\
& \oint_{c} H \cdot \hat{l} d l=\frac{\partial}{\partial t} \oiint_{s} D \cdot \hat{n} d S
\end{aligned}
$$

where $c$ bounds the surface $S$ and $\hat{n}$ is the normal unit vector to the differential elemental area $d S$ as shown in Figure 2.1. As $\Delta h$ approaches zero while $\Delta l$ is kept small B and D become finite. Also the R.H.S. of the equations $(2.3 \& 2.4)$ approaches zero due to the reduction in area. Hence the above equations approach to

$$
\begin{aligned}
& \left(E_{\mathrm{tan}}\right)_{1} \Delta l-\left(E_{\mathrm{tan}}\right)_{2} \Delta l=0 \\
& \left(H_{\mathrm{tan}}\right)_{1} \Delta l-\left(H_{\mathrm{tan}}\right)_{2} \Delta l=0
\end{aligned}
$$

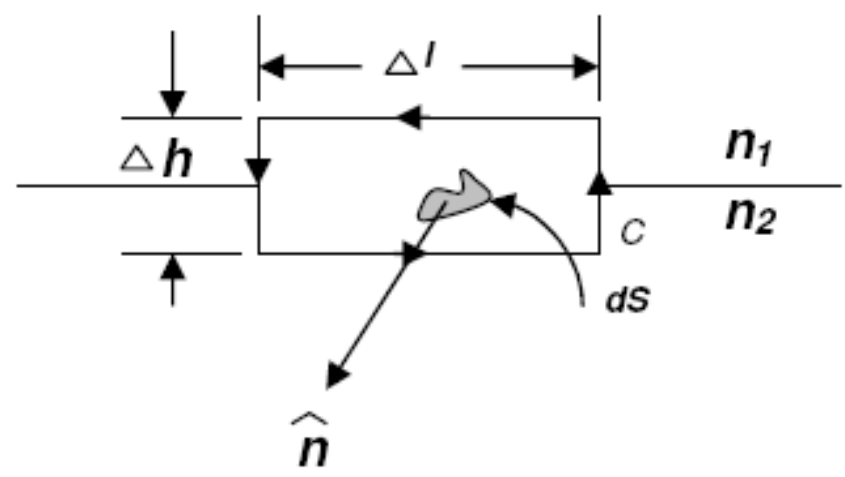

Figure 2.1 Dielectric Interface at region 1 and 2.

From the above equations we observe that the tangential electric and magnetic field components should be continuous at the interfaces.

\subsubsection{Phase Matching Conditions}

Consider the incident, reflected and the transmitted plane waves have propagation constants of $k_{i}, k_{r}$ and $k_{t}$ respectively. Then the corresponding waves can be written as 


$$
\begin{aligned}
& E_{i}(r)=A_{i} e^{-j\left(k_{i}, r\right)} \\
& E_{r}(r)=A_{i} e^{-j\left(k_{r}, r\right)} \\
& E_{t}(r)=A_{i} e^{-j\left(k_{t}, r\right)}
\end{aligned}
$$

According to Eq (2.5) the tangential electric fields should be continuous across the boundary, thus we get

$$
E_{i}(0, y, z)+E_{r}(0, y, z)=E_{t}(0 y, z)
$$

The only non trivial solution for the above solution is that $k_{i y}=k_{r y}=k_{t y}$ and $k_{i z}=k_{r z}=k_{t z}$. From the equations it can be inferred that the propagation constants along the tangential direction are maintained constant at interfaces. In Figure 2.2 the propagation constants remain same along $\mathrm{y}$ and $\mathrm{z}$ directions, these relations are known as phase matching relations [20].

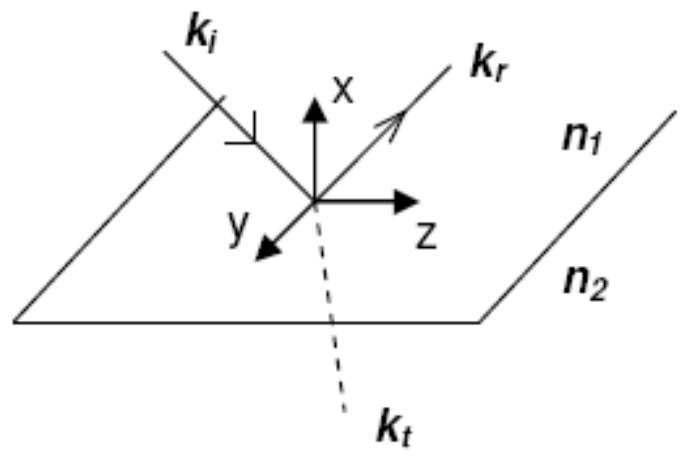

Figure 2.2 Dielectric Interface at region 1 and 2

\subsection{Guided wave optics}

This concept originated in the 1800 s with the demonstration of total internal reflection by John Tyndall. This chapter presents the basic theory of optical waveguides. Optical guiding technique has been developed and applied to establish secure communications and construct extremely small size optical and optoelectronic devices requiring the restriction of light. Optical waveguides transmit light from one location to another, the principle of optical confinement is based on the properties of a medium of one refractive index enclosed in a medium of lower refractive index, and the light is trapped by multiple total internal reflections. Basically an optical waveguide consists of a slab, strip or a cylinder of two dielectric materials of different refractive indices. The optical fiber waveguide geometry is most widely used which consists of two concentric 
cylinders of low-loss dielectric material. For integrated devices, slab and rectangular waveguide geometries are most applicable.

\subsubsection{Planar Mirror Waveguides}

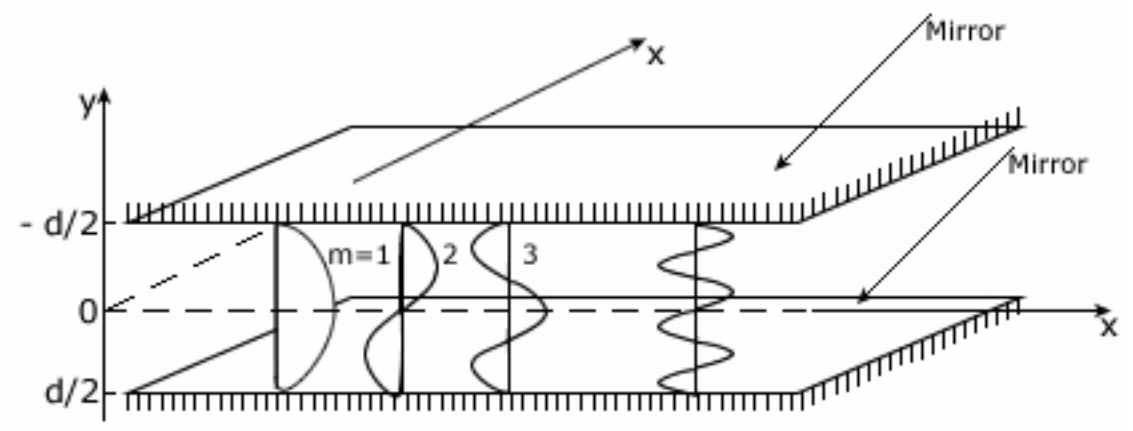

Figure 2.3 propagation of wave in a Planar Mirror waveguide

Figure 2.3 shows the wave propagation in a waveguide made of two parallel infinite planar mirrors separated by distance $d$, assuming reflection without loss, the ray is making an angle $\theta$ with the mirrors and the ray is guided along the z-direction.

Modes are fields that maintain the same transverse distribution and polarization at all positions along the waveguide axis.

Monochromatic TEM plane wave of wavelength $\lambda=\frac{\lambda_{o}}{n}$, wave number $k=n k_{o}$ and phase velocity $c=\frac{c_{o}}{n}$

Modes are fields that maintain the same transverse distribution and polarization at all distances along the waveguide axis.

$$
\begin{gathered}
\frac{2 \pi}{\lambda} 2 d \sin \theta=2 \pi m, m=1,2, \ldots, \\
m=q+1 \\
\sin \theta_{m}=m \frac{\lambda}{2 d} \quad m=1,2, \ldots . \rightarrow \text { Bounce angles }
\end{gathered}
$$

Each integer $m$ corresponds to a bounce angle $\theta_{m}$, and the corresponding filed is called the $m^{\text {th }}$ mode.

\section{Transverse component of wave vector}


The y component of the propagation constant is $k_{y}=n k_{o} \sin \theta$, is quantized to the values

$$
\begin{gathered}
k_{y m}=n k_{o} \sin \theta_{m}=\left(\frac{2 \pi}{\lambda}\right) \sin \theta_{m} \\
k_{y m}=m \frac{\pi}{d} \quad m=1,2, \ldots . .
\end{gathered}
$$

\section{$\underline{\text { Propagation Constants }}$}

Propagation constant for an electromagnetic field mode varying sinusoidally with time at a given frequency, is the logarithmic rate of change with respect to distance in a given direction of the complex amplitude of any field component.

The propagation constant, $\gamma$ is a complex quantity given by,

$$
\gamma=\alpha+i \beta
$$

where

$\alpha$, the real part, is the attenuation constant

$\beta$, the imaginary part, is the phase constant, and $i=\sqrt{-1}$

Propagation constant of the guided wave is

$$
\begin{gathered}
\beta=k_{z}=k \cos \theta, \beta \text { is quantized to the values } \beta_{m}=k \cos \theta_{m} \\
\Rightarrow \beta_{m}{ }^{2}=k^{2}\left(1-\sin ^{2} \theta_{m}\right) \\
\Rightarrow \beta_{m}{ }^{2}=k^{2}-\frac{m^{2} \pi^{2}}{d^{2}}
\end{gathered}
$$

The above equation tells us that the higher-order modes travel with smaller propagation constants.

\section{Number of modes}

Since $\sin \theta_{m}=\frac{m \lambda}{2 d}, m=1,2, \ldots$. and for $\sin \theta_{m}<1$, the maximum allowed value of $m$ is the greatest integer smaller than $(\lambda / 2 d)^{-1}, M=\frac{2 d}{\lambda}, \mathrm{M}$ is the number of modes.

Light can be transmitted through the waveguide in one, two or many modes. The actual number of modes that carry optical power depends on the source of excitation. The wavelength $\lambda_{\max }=2 d$ is called the cutoff wavelength of the waveguides. 
Cutoff frequency is defined as the lowest frequency of light that can be guided by the waveguide. Cutoff wavelength is the longest wavelength that can be guided by the structure and the corresponding cutoff frequency [26] is given by $v_{\min }=\frac{c}{2 d}$.

\subsubsection{Symmetric slab dielectric waveguide}

The slab of dielectric material is surrounded by media of lower refractive indices. Light is guided by total internal reflection inside the slab. In thin film devices the slab is called the film or waveguiding layer; the upper and lower media are called the cover or superstrate and the substrate.

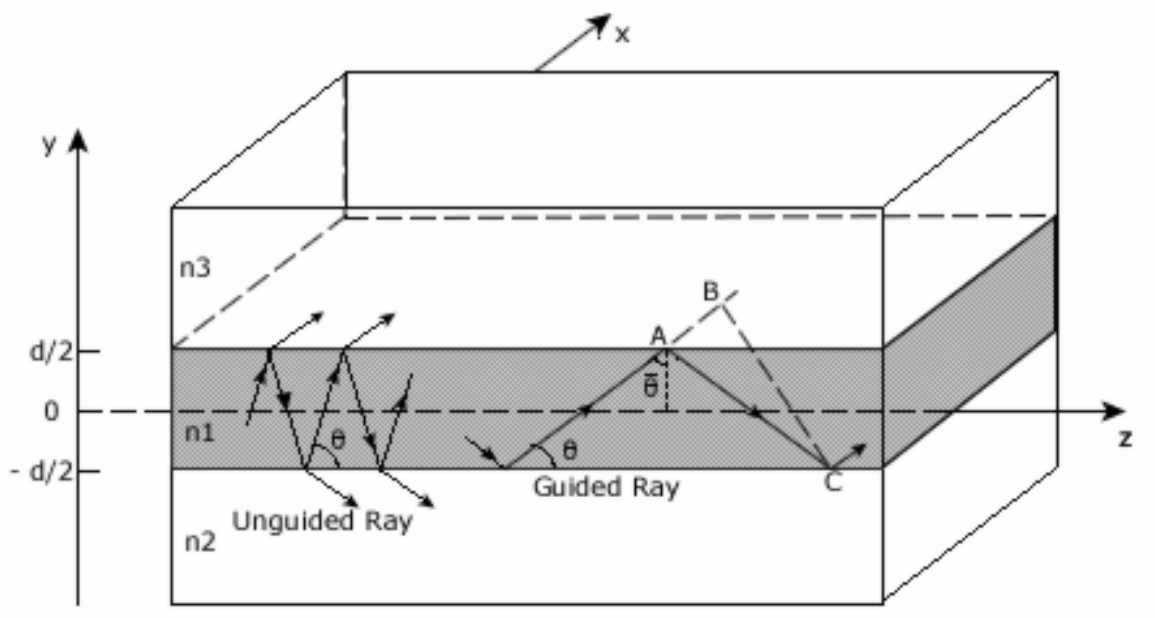

Figure 2.4 shows the waves traveling along a symmetric slab dielectric waveguide

If $\mathrm{n}_{2}>\mathrm{n}_{1}$ for traveling waves at an angle with respect to the normal to the interface

(x) greater than the critical angle $\theta_{c}=\sin ^{-1} \frac{n_{2}}{n_{1}}$, then this acts as waveguide. Not all angles greater than the critical angle will correspond to a guided wave and the guided electromagnetic fields are limited as modes of the guides.

The tangential electric $(\bar{E})$ and magnetic $(\bar{H})$ fields should be continuous across the boundaries.

Transverse electric mode (TEM) with its polarization in the y-direction and tangential to the interface is considered.

With $E_{y}$ there is an orthogonal $\bar{H}$ field whose z component will be the tangential component. 
Using Maxwell's equation for $\bar{E}$

$$
\begin{aligned}
& \nabla \times \bar{E}=-\frac{\partial \bar{B}}{\partial t}=j \omega \mu \bar{H} \\
& \Rightarrow \overline{H_{z}}=\frac{j}{\omega \mu} \frac{\partial}{\partial x} E_{y}(x, z)
\end{aligned}
$$

$k_{x}$ must be imaginary

$$
k_{1 x}=j \sqrt{{k_{z}^{2}-k_{1}^{2}}^{2}}=j \sqrt{k_{2} \sin ^{2} \theta-k_{1}^{2}}=j k_{o} \sqrt{n_{2}^{2} \sin ^{2} \theta-n_{1}^{2}}= \pm j \alpha .
$$

This is the evanescent field just outside the guide exponentially decaying over a length scale represented by $\alpha^{-1}$. The field propagates as $\exp \left(j k_{z} z\right)$ in the $z$ direction everywhere, $k_{z}$ is $\beta$ the propagation constant.

Within the guide there are positive and negative traveling waves in the $\mathrm{x}$ direction.

$A_{2} \cos \left(k_{2 x} x\right)$ for symmetric modes (even)

$A_{2} \sin \left(k_{2 x} x\right)$ for antisymmetric modes (odd parity)

$$
k_{2 x}=\sqrt{k_{2}^{2}-\beta^{2}}
$$

Electric Fields in Guide

$$
E_{y}=A_{1} \exp (-\alpha x)
$$

\subsubsection{Analysis of Symmetric Slab Dielectric Waveguides}

Assuming that the waveguide extends to infinity (usually ten wavelengths) along the $y$ direction. Since it is symmetric waveguide geometry we have the same refractive indices on both sides of the waveguide. The equation in each region can be written as [22]

$$
\begin{gathered}
\frac{\partial^{2} E(x, y)}{\partial x^{2}}=\left(k_{2} n_{1}^{2}-k_{z}^{2}\right) E(x, y)=0 \quad \text { Cladding } \\
\frac{\partial^{2} E(x, y)}{\partial x^{2}}=\left(k_{2} n_{2}^{2}-k_{z}^{2}\right) E(x, y)=0 \quad \text { Waveguide }
\end{gathered}
$$

The solutions to the above equations describe the propagation of light in both the mediums and are called modes. A mode is a spatial distribution of optical energy in one or more dimensions [22]. A guided mode is a set of electromagnetic fields which 
maintain their transverse spatial distribution along the propagating direction. A guided wave consists of a non polar wave decaying in $\pm x$ direction in the cladding region while maintaining a propagation constant of $k_{z}$ in the $z$ direction. The electric field component in these regions is

$$
\begin{aligned}
& E_{y}(x)=C_{1} \exp \left(-\alpha_{1} x\right) \quad x>d / 2 \quad \text { Cladding } \\
& =C_{2} \cos \left(k_{2 x} x\right) \quad-d / 2<x<d / 2 \quad \text { Waveguide region } \\
& =C_{1} \exp \left(\alpha_{1} x\right) \quad x>d / 2 \quad \text { Substrate } \\
& \text { Where } \alpha_{1}=\sqrt{k_{z}^{2}-\omega^{2} \mu \varepsilon_{1}} \text { and } k_{2 x}=\sqrt{\omega^{2} \mu \varepsilon_{2}-k_{z}^{2}} \text {. }
\end{aligned}
$$

$\mathrm{C}_{1}$ is used on both sides of the waveguides due to symmetry and this would change if the refractive indices on the two sides of the waveguide are different. A cosine wave in the waveguide region would give us even modes while sine wave would give us odd modes.

\subsection{The Prism Coupler}

Optical power can be coupled into or out of a slab waveguide by the use of a prism. The prism-film coupler is as device used to couple a laser beam efficiently into thin-film dielectric wave guides. One of the main processes for examining the propagation characteristics of dielectric waveguides is the prism coupler. It is based on the principle of distributed coupling through evanescent fields to the modes of thin-film waveguides. The prism is placed above the thin-film guide and is separated from it by a small gap of low refractive index this incident light is totally reflected at the base of the prism, and the waves in the fields in the prism and in the film are coupled through their evanescent fields in the gap [27]. The simple prism coupler consists of a high-refractiveindex prism placed close to a slab dielectric waveguide, the optical beam passing through the prism is incident upon its bottom at an angle greater than critical angle and from the base of the prism the evanescent fields capable of transferring power (between the incident beam and a waveguide mode) penetrate into the waveguide [28]. Also, using prism coupler we can determine the refractive index and thickness of the guiding film by measuring the coupling angles at the prism and fitting them to the dispersion curve, 
provided that the film supports two or more modes. To analyze the operation of the prism coupler it is important to understand the phenomena of frustrated total internal reflection.

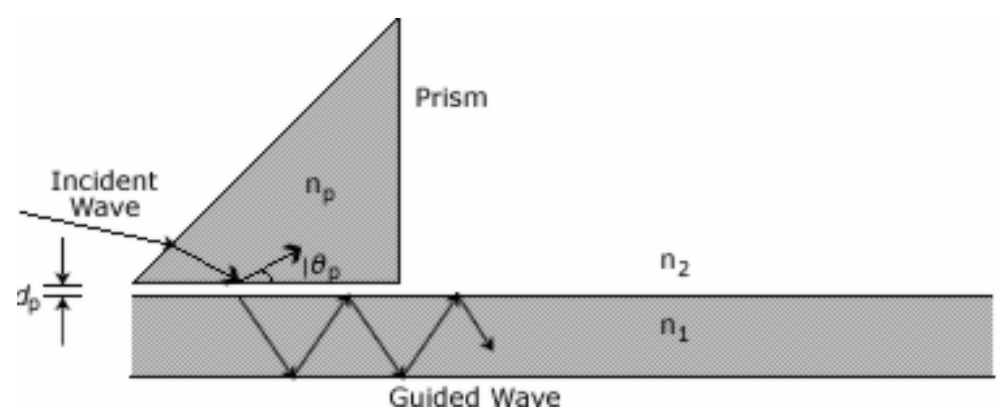

Figure 2.5 Prism coupler coupling light into slab waveguide

$n_{p}>n_{2}$ is placed at a distance $d_{p}$ from the slab waveguide of refractive index $n_{l}$.

Rays traveling in each mode angle have a corresponding modal index which is also known as effective index. This is given by [28]

$$
N(\theta)=n_{p} \sin \left(\theta_{1}\right)
$$

where $n_{p}$ is the refractive index of the prism and $\theta_{1}$ is the angle it makes with the base of the prism. More generally the effective index is $\operatorname{simply} n \sin (\theta)$ where $\theta$ is the angle of incidence the light makes at the boundary of the material. Since from Snell's law

$$
n_{1} \sin \left(\theta_{1}\right)=n_{2} \sin \left(\theta_{2}\right)
$$

where $n_{1}, \theta_{1}$ are refractive index and angle of incidence in medium 1 respectively and the same with the other medium. When the rays enter the waveguide it obeys the Snell's law. Hence the quantity $n_{p} \sin (\theta)$ remains the same and this quantity is maintained for all the transitions. Hence we get

$$
n_{p} \sin \left(\theta_{1}\right)=n_{w g} \sin \left(\theta_{2}\right)
$$

From the above expression we can conclude that the value $N(\theta)$ remains the same. During modal angles the light enters into the waveguide and the corresponding $N(\theta)$ is said to be the effective refractive index ( $\left.\mathrm{n}_{\text {eff }}\right)$ of the guiding wave.

An optical wave is incident into the prism such that it undergoes total internal reflection within the prism at an angle $\theta_{\mathrm{p}}$. the incident reflected waves form a wave traveling in the $\mathrm{z}$-direction with a propagation constant $\beta_{p}=n_{p} k_{o} \cos \theta_{p}$. The transverse 
field decays exponentially in the space separating the prism and the slab extending outside the prism.

The wave is coupled to a mode of the slab waveguide with a matching propagation constant $\beta_{m} \approx \beta_{p}$, if the distance $d_{p}$ is small. Power can be coupled into the waveguide with the appropriate interaction distance.

\subsection{Coupled Mode Theory (CMT)}

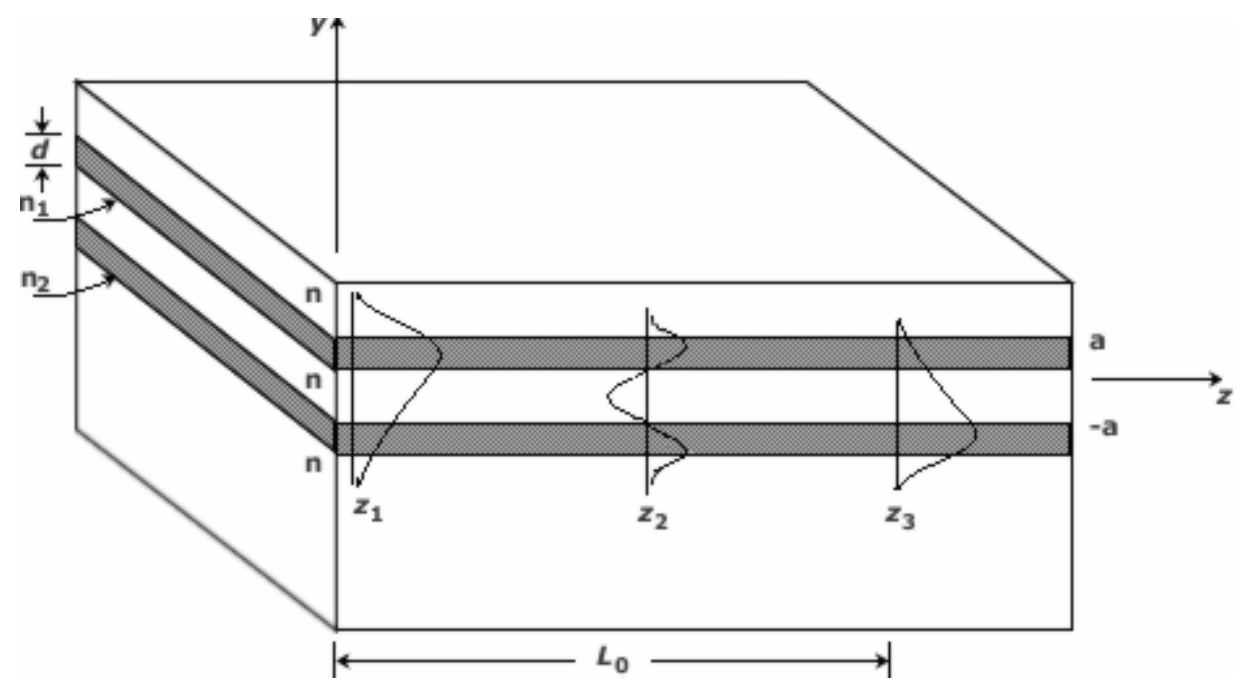

Figure 2.6 Coupled waveguides

Optical power can be transferred between the waveguides, if fields of the two waveguides overlap then light can be coupled from one into the other. Coupled mode theory explains this behavior of coupled waveguides. The SPARROW biosensor has two resonant waveguides and the waveguides are in resonance and based on coupled mode theory.

Coupled mode theory has been applied to analyze the electromagnetic wave propagation in integrated optics and the propagation parameters of an electromagnetic wave in the waveguide changes at perturbations. The analysis of coupled waveguide system is based on the modes, propagation constants and amplitudes of the individual waveguides [26]. If the mode profiles are independent of each other (orthogonal) then orthogonal coupled theory applies, else it is nonorthogonal and the solution is obtained for the both cases from the linear superposition of the waveguide modes [30]. If the 
device consists of weakly coupled waveguides, similar propagation constants then orthogonal coupled mode theory can be applied.

Consider two guided modes which have the amplitude $a_{1}$ and $a_{2}$ respectively with implicit time dependence $\exp (j w t)$. If they are infinitely apart from each other then they would obey the equations [30]

$$
\frac{d a_{1}}{d z}=-j \beta_{1} a_{1} \quad \frac{d a_{2}}{d z}=-j \beta_{2} a_{2} \quad 2.30
$$

where $\beta 1$ and $\beta 2$ are the propagation constants and will remain the same. If they are brought close, the evanescent fields would interact with each other and hence mode coupling occurs. If the coupling is weak the equation would be

$$
\begin{aligned}
& \frac{d a_{1}}{d z}=-j\left(\beta_{1}+K_{11}\right) a_{1}-j K_{12} a_{2} \\
& \frac{d a_{2}}{d z}=-j\left(\beta_{2}+K_{22}\right) a_{2}-j K_{21} a_{1}
\end{aligned}
$$

where $\left(\mathrm{K}_{12}, \mathrm{~K}_{21}\right)$ and $\left(\mathrm{K}_{11}, \mathrm{~K}_{22}\right)$ are the mutual and self coupling coefficients respectively. If the waveguides are lossless then the total power has to be determined constant. Hence,

$$
P(z)=\left|a_{1}\right|^{2}+\left|a_{2}\right|^{2}
$$

From the principle of power conservation we get

$$
\frac{d}{d z} P(z)=0
$$

Solving the above equation 2 . we get

$$
K_{12}=K_{21}^{*}=\kappa
$$

For the uniform couplers both the propagation constants and the coupling coefficients are independent of $\mathrm{z}$ and hence equations 2.4 can be solved analytically. For simplification we assume

$$
a_{i}(z)=\hat{a}_{i} \exp \left(-j \frac{\beta_{1}+K_{11}+\beta_{2}+K_{22}}{2} z\right)
$$

Here Eq. 2.30 can be reframed as

$$
\frac{d \hat{a}_{1}}{d z}=-j \delta \hat{a}_{1}-j \kappa \hat{a}_{2}
$$




$$
\begin{gathered}
\frac{d \hat{a}_{2}}{d z}=-j \delta \hat{a}_{2}-j \kappa \hat{a}_{1} \\
\text { Where } \delta=\frac{\beta_{1}+K_{11}-\beta_{2}-K_{22}}{2}
\end{gathered}
$$

Rewriting the above in the matrix form we get

$$
\begin{gathered}
\frac{d}{d z} A=-j \bar{H} A \\
\text { Where } A=\left[\begin{array}{l}
\hat{a}_{1} \\
\hat{a}_{2}
\end{array}\right]
\end{gathered}
$$

And $H=\left[\begin{array}{ll}+\delta & \kappa \\ \kappa & -\delta\end{array}\right]$ After diagnolization by unitary matrix such that $\mathrm{O}^{\mathrm{t}} \mathrm{HO}=\mathrm{B}$ where $\mathrm{B}$ is the diagonal matrix the equation obtained is

$$
\left[\begin{array}{ll}
\beta_{s} & 0 \\
0 & \beta_{a}
\end{array}\right]
$$

where $\beta_{\mathrm{s}}$ and $\beta_{\mathrm{a}}$ are the symmetric and antisymmetric modes of the combined structure. Thus the beta values of the composite modes can be written as

$\beta_{s}=\beta_{o}+S$ and $\beta_{a}=\beta_{o}-S$ where $S=\sqrt{\delta^{2}+\kappa^{2}}$ and $\beta=\frac{\beta_{1}+K_{11}+\beta_{2}+K_{22}}{2}$.

Assuming only one waveguide is excited then $\mathrm{a} 1=1$ and $\mathrm{a} 2=2$, the power in waveguides can be written as

$$
P_{1}(z)=\cos ^{2}(S z)+\cos ^{2}(\eta) \sin ^{2}(S z) \quad P_{2}(z)=\sin ^{2}(\eta) \sin ^{2}(S z)
$$

where

$$
\eta=\tan ^{-1}\left(\frac{\kappa}{\delta}\right)
$$

At $\mathrm{z}=\mathrm{L}_{\mathrm{c}}$ maximum power transfers from waveguide 1 to waveguide 2 and this $\mathrm{L}_{\mathrm{c}}$ is known as coupling length and is given by,

$$
L_{c}=\frac{\pi}{2 S}
$$

Coupling length is the distance at which maximum power transfer occurs from one waveguide to the other waveguide. If the coupling length is higher the power transfer rate is slow and with lower coupling length the device would be prone to noise. 
Depending on the coupling length a suitable interaction length for sensing would be selected. A higher interaction length and lower coupling lengths would result in lot of power transfers from one waveguide to other and may result in higher losses.

\subsection{Loss Mechanisms in Optical Waveguides}

In a planar waveguide the propagation losses are due to three mechanisms
(1) Scattering
(2) Absorption
(3) Radiation

These losses depend on the waveguide geometry, its internal structure, and the source wavelength. In an amorphous waveguide the scattering losses is a combination of both interface scattering and volume scattering. Interface scattering is due to the interface roughness, and the volume scattering is due to the local fluctuations in the refractive index that result from density and compositional variations [25]. Scattering loss predominates in glass or dielectric waveguides. Absorption losses are negligible compared to scattering losses and are more important in semiconductor and other crystalline materials as the absorption is caused by three different mechanisms, 1 . Absorption by atomic defects in the glass composition, 2. Extrinsic absorption by impurity atoms in the glass material, 3. Intrinsic absorption by the basic constituent atoms of the fiber material ${ }^{1}$. If contaminant atoms such as hydrogen are present then the absorption losses are present. Radiation losses are significant in the bent waveguides.

Number of reflections from each surface: $\quad N_{R}=\frac{L}{2 t_{g} \cot \theta_{m}}$ $I(z)=I_{o} e^{-\alpha z}$

\section{Coupling Losses:}

When the light is coupled into the waveguide the losses in the waveguide correspond to the coupling loss. These losses can be minimized by using efficient couplers and also depend upon the optical sources employed to input light into the waveguides. Coupling losses are independent of the waveguide parameters and depend on the operation of the device. When using prism coupler to couple the light into the 
waveguide maximum power can be transferred with minimum loss by coupling the light at the coupling spot.

All the above mentioned losses can be neglected for the optical dielectric waveguides as they are all negligible except the scattering losses for loss measurements.

\subsection{Thin Film Fabrication}

\begin{tabular}{|l|l|}
\hline Property & Application \\
\hline Optical & $\begin{array}{l}\text { waveguides, antireflective } \\
\text { coatings }\end{array}$ \\
\hline Electrical & $\begin{array}{l}\text { insulating barriers, } \\
\text { semiconductors }\end{array}$ \\
\hline Mechanical & hardness, adhesion \\
\hline Chemical & $\begin{array}{l}\text { protective barriers against } \\
\text { oxidation of diffusion }\end{array}$ \\
\hline Magnetic & magnetic disks \\
\hline Thermal & heat sinks \\
\hline
\end{tabular}

Table 2.1 shows the applications of thins films in various fields [31]

Our group uses state of the art Ion source assisted Electron Beam evaporator for the fabrication of thin dielectric films for the SPARROW Biosensor.

Electron beams are often required for the depositions of thin films from materials which vaporize above $1500^{\circ} \mathrm{C}$. In electron beam deposition a filament is held between $10-50 \mathrm{kV}$ below a copper hearth which contains the source material. A small potential is applied across the filament until enough current is passing through it so that electrons are thermionically emitted from it. The electrons are accelerated through the potential difference and steered towards the source material by magnetic deflection. The high energy electron bombardment can result in secondary electron emission from the source material leaving it with a positive charge after the electrons are quickly drawn towards ground. In dielectric substrates charge can build due to these positive ions and begin to repel ionized source material from the substrates surface [29]. Also the high energy 
electrons can generate $\mathrm{x}$-rays when they strike the source material damaging the depositing films and substrates [31]. 


\section{SPARROW BIOSENSOR Modeling and Fabrication}

This chapter gives the overview of the complete process for building the SPARROW sensor. The procedure in building the SPARROW sensor involves the following steps: Modeling and designing, fabrication of coupled waveguides according to the design, optical characterization, loss measurements, silicon master fabrication for microfluidic channels, and growth of bio-layer.

\subsection{Process Flow}

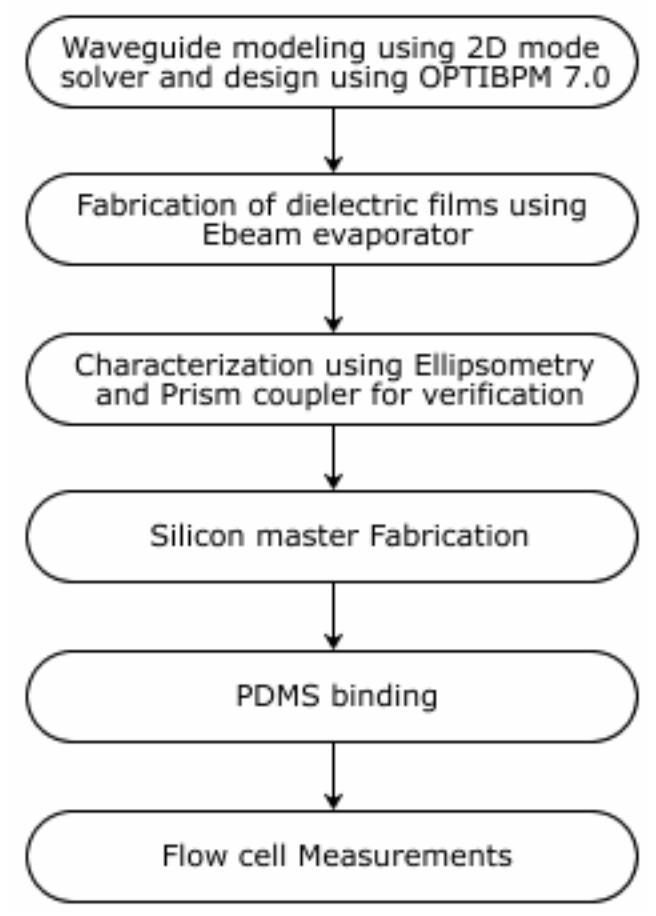

Figure 3.1 Process flow of experiment

\subsection{Modeling BPM 2D Mode Solver}

As mentioned in Chapter 1 our model of the SPARROW biosensor is based on the evanescent wave technique. The first part of the process with the designing of the sensor using Mode Solver 2D and BPM 2D for the simulations using higher order Padé approximations gave relatively accurate results. The BPM 2D simulator is based on the unconditionally stable finite difference method algorithm of Crank-Nicolson.

Modes in a waveguide are dependent on various parameters such as:

1. Wavelength of incident light: decreasing the wavelength will increase the number of supported modes. 
2. Thickness of the waveguides: increasing thickness will increase the number of supported modes.

3. Index of the waveguides: increasing the index of waveguides will increase the number of supported modes.

Based on this, the optical mode profile in dielectric slab waveguides is dependent on the thickness and refractive index of the waveguide and also on the index contrast between the waveguide and cladding or substrate.
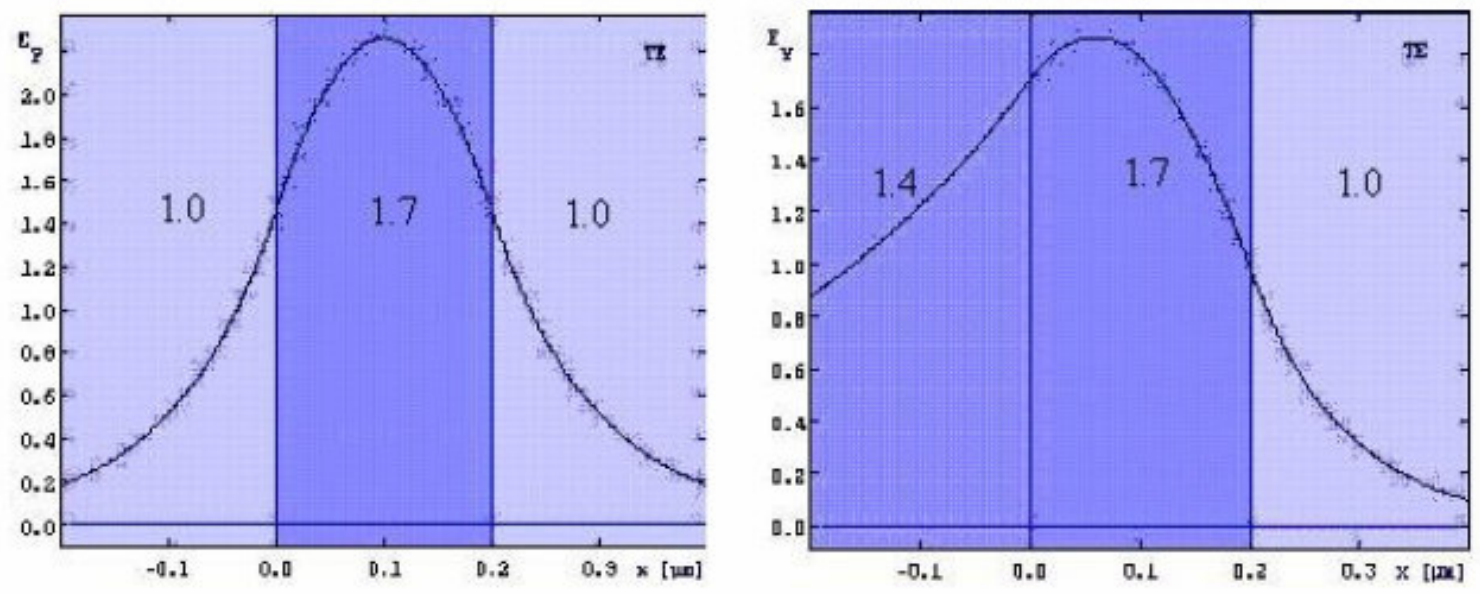

Figure 3.2 shows the mode profile of waveguide

Figure 3.2 shows the modal profile of the structure having a substrate waveguide, waveguide layer \& a cladding layer. The figure to the left is a symmetric waveguide with $200 \mathrm{~nm}$ thickness and refractive index of 1.7 and the refractive index of both substrate \& cladding layer is 1 . It is clear from the figure that the mode profile is symmetric with the same penetration depth (defined as 1/e distance from the waveguide surface) is $53 \mathrm{~nm}$ on both sides, whereas for an asymmetric waveguide shown in Figure 3.2 to the right the penetration depth is not the same on both sides. It is $146 \mathrm{~nm}$ on the higher index side and $40 \mathrm{~nm}$ on the lower index side (1.0). It can be inferred from the optical field intensity in the above figure that high index contrast results in abrupt drop off in intensity and low penetration depth.

The general model of the SPARROW sensor is shown in Figure 3.3 with penetration depth shown. 


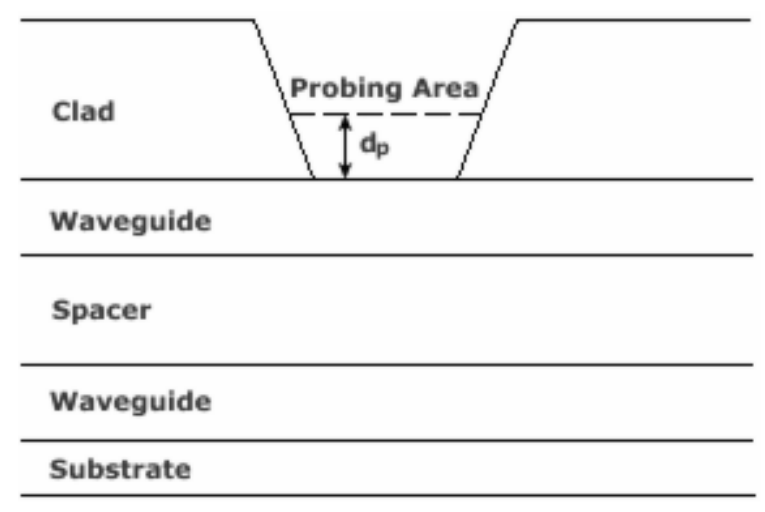

Figure 3.3 Probing area

In the figure $d_{p}$ is the penetration depth also called as probe depth. This depth is composed of the analyte with bioagents to be detected. The penetration or probe depth depth over the area swept out by the guided beam represents a detection volume in which the device can sense changes in refractive index. Depending upon the organic chain selected and the bio-agent, the length of the biolayer can vary from $40 \mathrm{~nm}-120 \mathrm{~nm}$, the molecule binds with the targeted analyte in solution within the biolayer. The ratio of the interaction volume of the bio-agents bonded to the biolayer to the evanescent wave modal volume in the region should be as high as possible for efficient operation of biosensor. It is very important for us to choose a biolayer with smaller length and this model is designed to give larger penetration depth.

In our device architecture with an evanescent tail of $100 \mathrm{~nm}$, a biolayer thickness of $40 \mathrm{~nm}$ gives us $60 \mathrm{~nm}$ of usable probe depth within the detection volume in which to detect targets.

The next step is to build and implement this model and run simulations to calculate power transfer and optical field variation in these coupled waveguides. We use the "OPTIBPM" simulation tool based on the Beam Propagation Method (BPM) to perform waveguide simulations.

The two methods for calculation of eigenvalues and eigenfunctions are beam propagation methods (BPM) and a coupled-mode theory (CMT) method [1]. Beam propagation method is reliable and accurate especially for nonuniform waveguides but with certain limitations. The number of points that can be considered in parameter space are limited due to the fact that both the methods are time consuming. BPM can be used to compute electric fields in optical waveguides without using normal-mode eigenfunctions. 
The Beam Propagation Method is adopted to solve Helmholtz equations or the parabolic wave equation in space in terms of near-field (spatial) and far-field (angular) properties of electric field, also can produce information related to the modal description of the field at every mesh point [32].

The simulations performed using this tool are faster than compared to FDTD (Finite Difference Time Domain). Paraxial approximations are assumed in BPM, so can be used for multi waveguide structure and structures where backside reflection is not considered. Backside reflections are not considered when working with waveguides and since the ideal device design does not have back reflections this simulation tool can be employed. For the tuned structures OPTIBPM 7.0 has been used. Effective indices and modal fields of guided modes in two dimensions can be calculated. Based on the transfer matrix (TMM) method in 2D for a multilayer planar structure the program generates the results. The two dimensions are the transverse dimension denoted as the $\mathrm{X}$-direction and the dimension of propagation of light is the Z-direction. The optical field at any point of propagation can be calculated using this software.

The Figure 3.3 is the design that we considered for study. Light is coupled into a waveguide which propagates in the same waveguide until it reaches the probe area. Under the presence of analyte solution in the probe area, at this point the power is exchanged periodically between the waveguides as the two waveguides are in resonance. Complete power transfer occurs under the probe area due to the refractive index of the analyte solution. Based on the power observed at the ends of the probe area waveguide the bio-agent target attached to the Biolayer probe can be detected.

In order to design the coupled waveguides using OPTIWAVE we need to create an input wave, this input wave can be produced in many ways for our simulations. Here we choose modal field as an input wave since we are using a prism coupler to couple light into waveguides. Details concerning the input types and assumptions considered in the simulation software can be found in [37].

As seen in the previous section and Figure 3.2, 2D Mode solver was used to obtain the modes of the simulated structure. This simulation tool 2D Mode solver solves for modes, for a defined structure and outputs the modal intensity, which can be used as input wave for the simulations. 


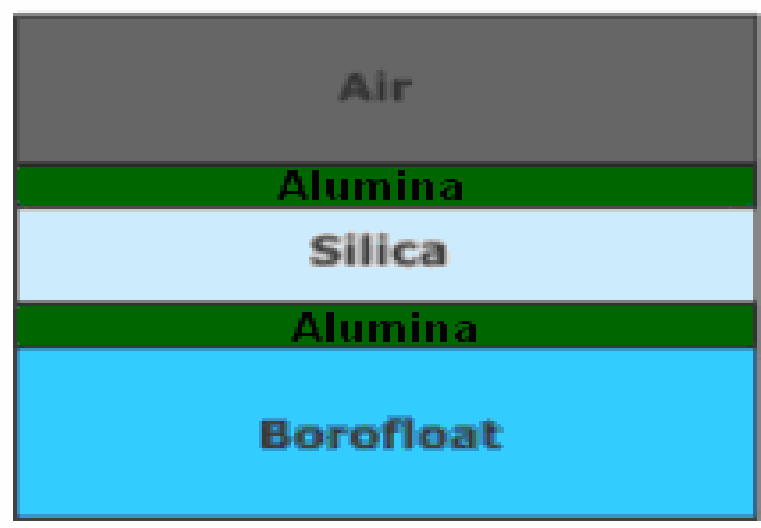

Figure 3.4a Simulation model for a sample structure

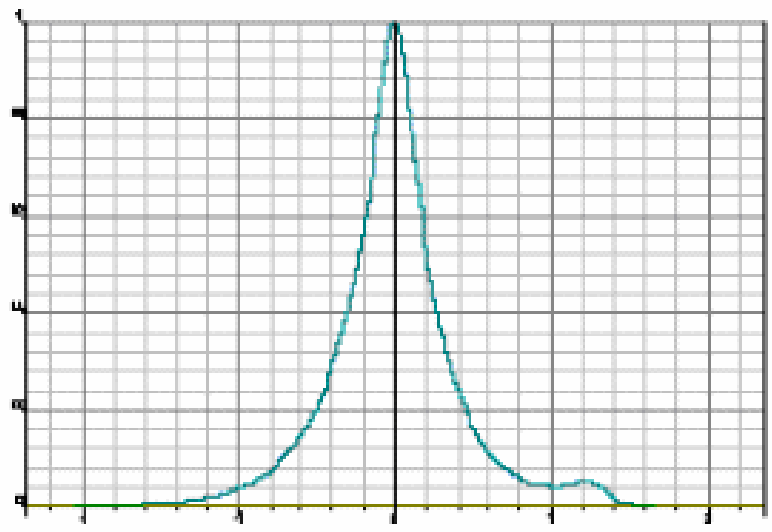

Figure 3.4b Optical Field Distribution.

Figure $3.4 \mathrm{~b}$ is the Optical field distribution for the model shown in Figure 3.4a, The 2D mode solver tool was used to obtain the optical field distribution. The output shows that most of the power is concentrated in the top waveguide for this structure with air as the cladding layer.

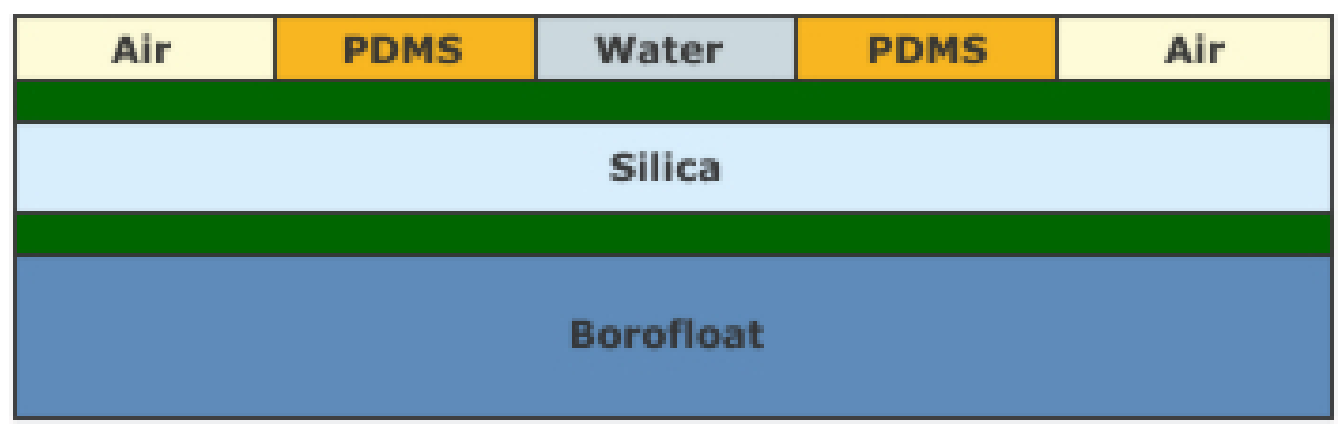

Figure 3.5 the model with the green color layers of Alumina for simulation in OPTIBPM 7.0 
It can be seen in the above figure that light interacts with all interfaces. The optical power transfer at the PDMS/air interface should be ideally zero and there should be complete power transfer or resonance under the probe area.

The biolayer grown on the exposed waveguide surface in the channel has an index of $1.4-1.5$ and the effective index when analyte is present (for our design water) can vary upwards from 1.3318 (DI water) depending on percentage coverage of the loaded biolayer. Therefore simulations have been performed assuming the channel index to vary from 1.3318 to 1.43 .

From the simulation results in Figure $3.4 \mathrm{~b}$ it is clear that the field should be confined to a single waveguide if the cladding layer is air or PDMS but should allow power transfer in the presence of analyte. Here our waveguides are designed to be tuned for an analyte with the refractive index of DI water. The simulated optical field variation for the above model in Figure 3.5 is show in Figure 3.6.

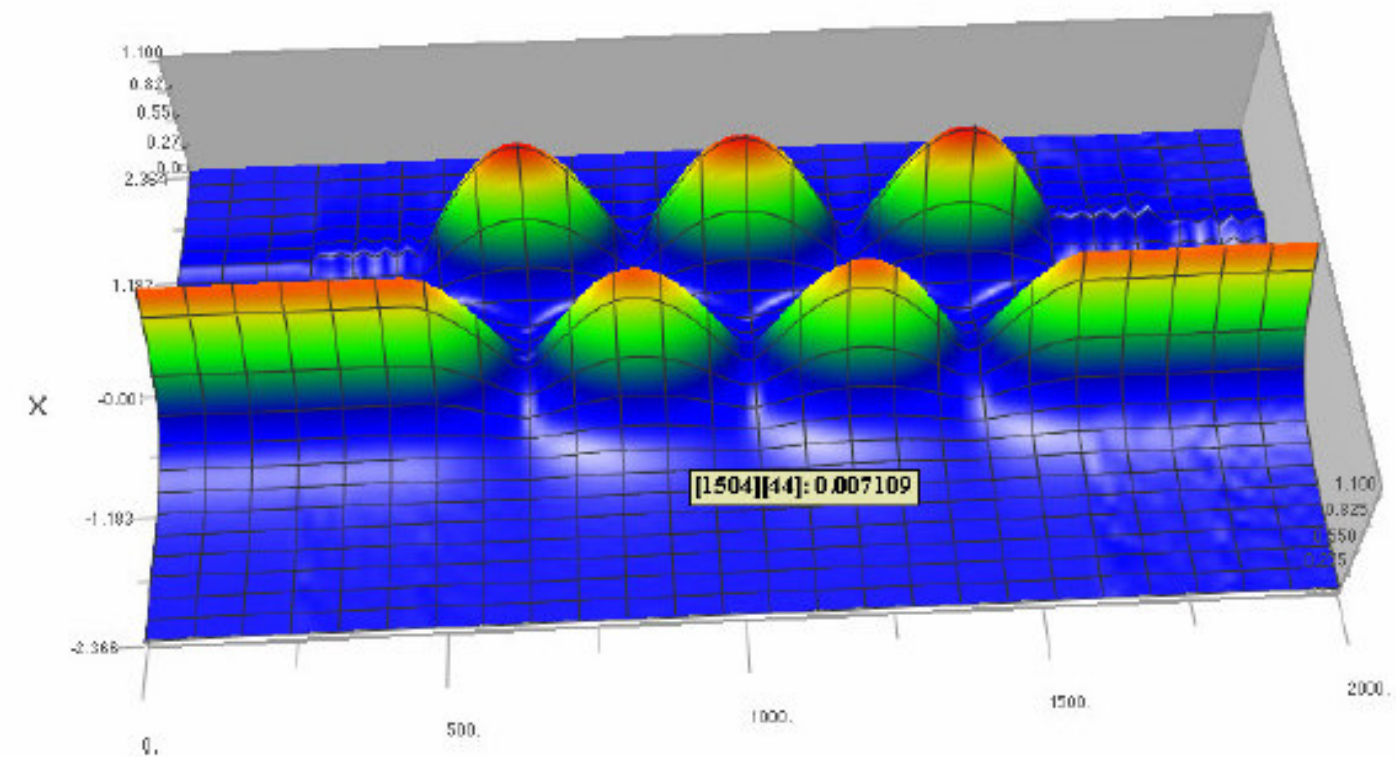

Figure 3.6 Field propagation in designed structure.

Figure 3.6 shows the simulated optical field intensity variation for the design in Figure 3.5. It can be observed that the optical power is confined to the bottom waveguide for air or PDMS and that there is complete power transfer at the water interface. Due to the sudden change in the medium there occurs scattering losses at the interfaces. The optical power observed at the ends of the waveguides is recorded and for a series of refractive indices of the analyte the simulations are performed, this power observed at the 
ends during simulations can be solved for using BPM simulations for finding the effective refractive index of the analyte present in the biolayer.

\subsection{Waveguide Film Stack Growth using Electron-Beam Evaporator}

In this section the growth of the film stack is described. For the deposition of thin films from materials above $1500^{\circ} \mathrm{C}$ often electron beams are required. In this method a filament is held between $10-50 \mathrm{kV}$ with the material placed below this filament in a copper hearth. When a small potential is applied across the filament electrons are emitted thermionically due to the current passing through the filament. These electrons are accelerated and directed towards the source material by magnetic deflection. The high energy bombardment results in secondary electron emission from the source material leaving it with a positive charge after the electrons are quickly drawn towards ground [33].

High index contrast waveguides are required for the biosensor structures, for this precise refractive index and thickness control are required. Refractive index variations of less than one percent are often necessary $[32,33]$. To fabricate such dielectric films the state of the art equipment, an Ion Beam Assisted Deposition (IBAD) source and electron-beam evaporator was used. Electron-beam evaporation is a type of physical vapor deposition (PVD) commonly used for oxide deposition [34]. This biosensor design consists of two resonantly coupled alumina waveguides as seen in Figure 3.1 of thickness less than 200 $\mathrm{nm}$ separated by a spacer layer of silicon dioxide of $1 \mu \mathrm{m}$ thickness approximately. The structure was carefully designed for desired thickness and refractive index for resonance, therefore precise control of nanoscale thickness and refractive indices are necessary. To address these fabrication challenges IBAD technology is used.

Aluminum oxide $\left(\mathrm{Al}_{2} \mathrm{O}_{3}\right)$ for the waveguides and Silicon dioxide $\left(\mathrm{SiO}_{2}\right)$ for spacer layer are the materials chosen for the biosensor. Aluminum oxide has higher index

of refraction of 1.65 approximately at $633 \mathrm{~nm}$ wavelength. Alumina waveguides offered stable physical (thickness and surface roughness) and optical (refractive index and loss) properties [23]. 
Silicon dioxide has the lowest index of approximately 1.46 at $633 \mathrm{~nm}$ wavelength. It is amorphous and transparent from the UV to the IR region of the spectrum [23]. Silicon dioxide has been extensively used in wave guiding applications.

\section{Substrate material:}

Silicon is the standard substrate material for IC fabrication, but glasses exhibit attractive dielectric and optical properties. Glass is also supplied in wafer form in different compositions such as quartz, fused silica and borosilicate glass. Since glass is transparent for visible light, it is particularly suited for optical propagation devices. Glasses are chemically inert and suitable for high temperature application. Highly polished Borofloat glass wafers were chosen as substrates due its low cost compared to quartz.

These depositions were performed on borofloat wafers which were cleaned in a five minute acetone and five minute methanol degreasing bath. In an attempt to achieve an understanding of the deposition parameters that were affecting the growths we performed numerous depositions under different conditions. Some trends were clearly seen, such as the fact that pressure inside the chamber at the beginning of the deposition had an effect on the resulting index which would be addressed in the next chapter.

\section{Aluminum Oxide from Electron-Beam Depositions}

The Aluminum dioxide deposition was performed at a deposition rate of $3.5 \AA / \mathrm{s}$, with substrate temperature starting with $200^{\circ} \mathrm{C}$ and increasing to about $245^{\circ} \mathrm{C}, 20 \mathrm{RPM}$ of substrate rotation, a neutralizer current of $21 \mathrm{~A}$, drive current of $1.3 \mathrm{~A}$, drive voltage around $155 \mathrm{~V}, 16 \mathrm{sccm} \mathrm{O}_{2}$ flow which lead to a chamber pressure of $5 \times 10^{-5}$.

\section{Reactive deposition of Silicon dioxide using IBAD}

The silicon dioxide deposition was performed at a deposition rate of $3.5 \AA / \mathrm{s}$, with substrate temperature starting with $200^{\circ} \mathrm{C}$ and increasing to about $275^{\circ} \mathrm{C}, 20 \mathrm{RPM}$ of substrate rotation, a neutralizer current of $21 \mathrm{~A}$, drive current of $1.3 \mathrm{~A}$, drive voltage around $170 \mathrm{~V}, 16 \mathrm{sccm} \mathrm{O}_{2}$ flow which lead to a chamber pressure of $5 \times 10^{-5}$. 


\subsection{Characterization Techniques}

The ability to accurately determine the chemical and physical properties of thin films is required for the optimization of the growth process. Thickness, refractive index, film intermix, surface roughness, stoichiometry, and material strain are common thin film characteristics of interest.

\subsubsection{Ellipsometry}

The samples fabricated by electron beam evaporator are taken out from the chamber for characterization. Spectroscopic ellipsometry measurements were done using a J. A. Woollam M-2000U Ellipsometer. By studying the polarization of light that is reflected off a surface, information can be gained about the properties of the reflective surface if the original polarization of the light was known.

- Surface analysis and grazing index can also be performed to check for the uniformity.

- The data is used to check with the designed model. If not the values are then given as input to the $2 \mathrm{D}$ mode solver to design new model and to set the tooling factor for the Ebeam.

\subsubsection{Prism Coupler and Effective Refractive Index [28]}

In chapter 2, Prism coupler and its principle was detailed. Frustrated Total Internal Reflection takes place between the prism and film interface allowing light to travel into the thin film.

Each mode in a waveguide depending upon the medium has a distinct angle at which it propagates. When light is incident onto the prism, total internal reflection takes place and there is also an evanescent wave going into the second medium. If the velocity of the evanescent waves matched with the phase velocity of a mode of the waveguide then the light propagation would be along the matched modal path. At these positions called mode angles an optical propagating mode develops in the waveguide and the reflected power is diminished. 


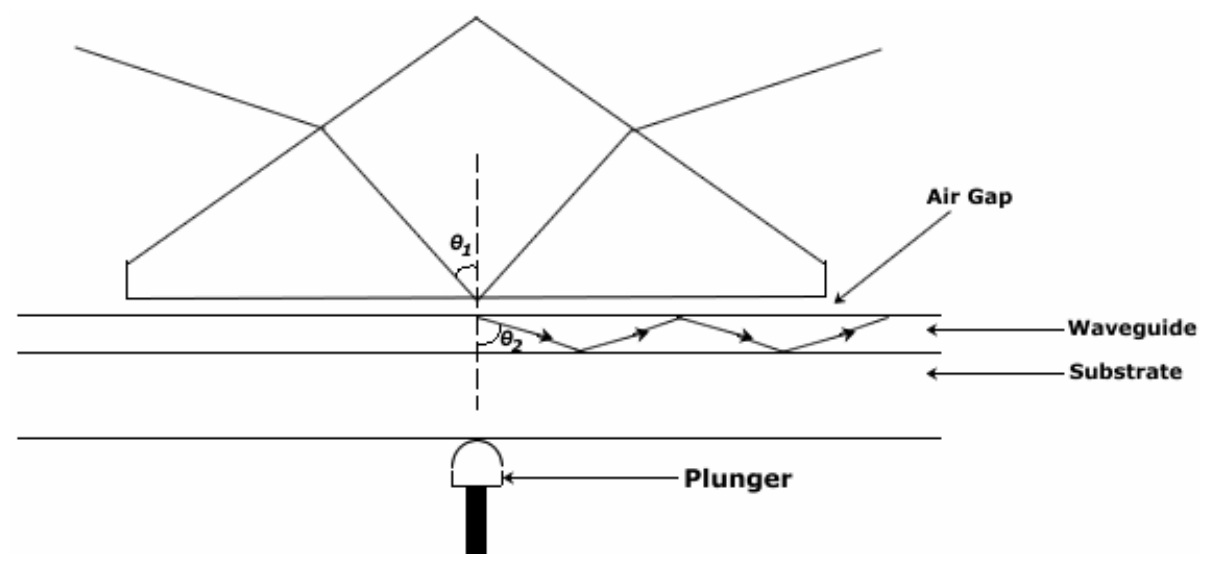

Figure 3.7 prism coupler setup and stack

The intensity plot obtained when the SPARROW based structure is checked for modes in the prism coupler. The distance between the film and the prism is less than the wavelength of light. Both TE and TM modes can be observed by polarizing the evanescent wave.

The modal equation is given by [28]

$$
(2 \pi / \lambda) \times \cos \left(\theta_{2}\right) \times d+\phi_{1}+\phi_{2}=m \pi \quad(m=0,1,2 \ldots),
$$

where $\lambda$ is the operating wavelength, $d$ is the thickness of the waveguide, $\phi_{1}$ and $\phi_{2}$ are the phase shifts at the air film interface and film substrate interface.

By knowing the two modal angles from the above equation parameters like thickness and index can be calculated. If only a single mode is present then one of the two parameters is required to calculate the other parameters.

The two commonly used techniques for coupling light into the waveguides are

- Prism coupler

- Butt coupler

Prism coupling based on FTIR causes minimum stress on the film. Mode sensitivity is possible using prism coupler but not through butt coupling. The device operation allows us to work with coupled waveguides and a single waveguide has to be excited at a time. Thus using the prism coupler a single mode can be excited. At an ' $\theta$ ' light couples into the waveguide at which the propagation velocity in the prism and air gap is and phase velocity equal corresponding to a mode in the waveguide and this angle serves to obtain the $\mathrm{n}_{\text {eff }}$ of the medium and can also be used to calculate the beta or the 
propagation constants of the waveguide. And this result is used as a reference to validate the results from the ellipsometry data and simulation models. Also loss measurements are done using prism coupler along with CCD camera.

\subsubsection{Prism Coupler and Loss measurement}

Metricon 2100 Prism coupling system, samples were held with a pneumatic plunger against a high index optical prism. A gadolinium garnet (GGG) prism of index 1.965 at $632.8 \mathrm{~nm}$ wavelength is used in this prism coupling system. Helium-neon laser producing a narrow polarizing beam of light at $632.8 \mathrm{~nm}$ wavelength was incident by inputting light at an alterable incident able to the prism.

Quantitatively the intensity at a point in the waveguide is given by

$$
I(z)=I_{o} e^{(-\alpha z)}
$$

where $\alpha$ is the attenuation coefficient and $\mathrm{I}_{0}$ is the intensity at $\mathrm{z}=0 . \operatorname{loss}$ in $\mathrm{dB} / \mathrm{cm}$ is given by $4.3^{*} \alpha$. This method includes all the losses and gives the overall loss of the waveguide. The evaluation of loss in the waveguides was performed using the video scan technique. A CCD camera setup with the prism coupler was used to capture the images. The optical quality of the waveguides is captured using this CCD camera and propagation loss is measured using digital image processing then curve fitting analysis was employed to fit the pixel intensity data and losses were calculated. More details given in Appendix B.

Example of single layer with loss calculation.

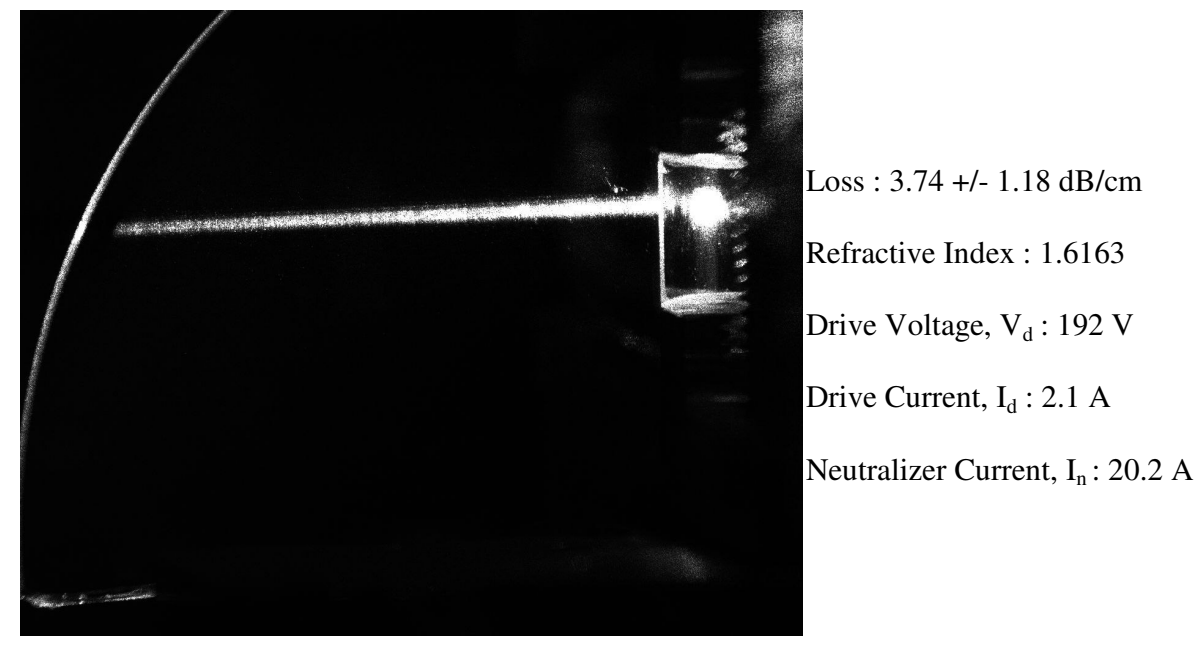

Figure 3.8 Single layer alumina film for loss calculation 


\subsection{PDMS channels and Silicon Master Fabrication}

Initially PMMA (Polymethyl Methacrylate) patterning was used to provide a channel for the analyte flow in combination with a flow chamber clamped to the sample. It was necessary to use a flow system that gave a more controlled flow environment for microfluidic addressing of the surface. Moreover, the channel needs to be able to withstand the treatments necessary for SAM and biolayer attachment..

Our group settled on a new arrangement for providing channels on the waveguide surface. PDMS (Polydimethyl siloxane) channels were fabricated using a silicon master and bonded to the upper alumina waveguide.

\subsubsection{Preparation of PDMS Channels}

To prepare PDMS channels first photo masks were needed. Photo masks were designed using "Freehand" and printed using a dot-matrix printer. They were then generated using photo plotting process with a resolution of 8000 dpi at WVU Printing Services. This mask making process is suitable for the resolution required for the channel. Nano SU8-25, negative photo resist is used to form the patterns on the Silicon master.

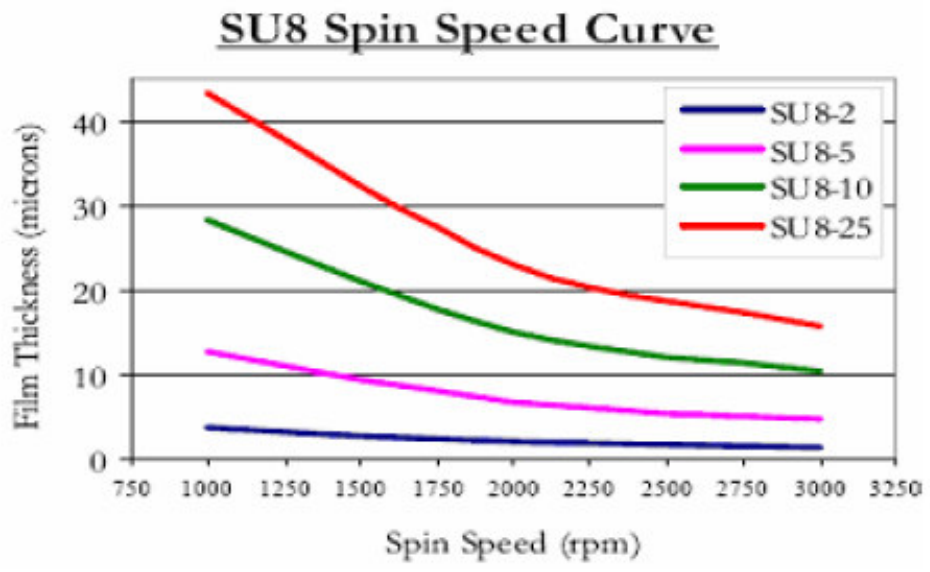

Figure 3.9 Spin speed curves for Nano SU8 [35]

\section{Nano SU 8 -25}

Nano SU8 is a negative photo resist. It is transparent over $360 \mathrm{~nm}$ and is good for obtaining features with good vertical sidewalls and thick films. 


\subsubsection{Process flow for Fabrication of Silicon Master}

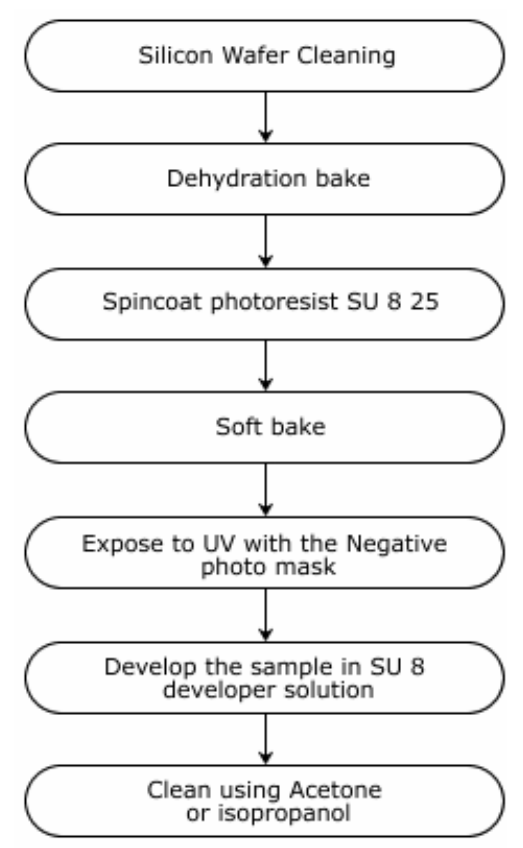

Figure 3.10 Process flow for silicon master fabrication and photomask

\section{Fabrication process:}

1. A general procedure for fabrication is spincoat, softbake, expose, post exposure bake and develop.

2. Lithography process:

3. A 3 inch silicon wafer is cleaned in acetone and methanol for 5 minutes each and blow dried using nitrogen.

4. The wafer is then baked in the oven to dehydrate for 10 minutes at $100^{\circ} \mathrm{C}$.

5. SU8 photo resist is spincoated on a spinner at

6. It is then soft baked on a hotplate at $65^{\circ} \mathrm{C}$ for 5 minutes and $95^{\circ} \mathrm{C}$ for 15 minutes.

7. The wafer is aligned with the mask and exposed to UV light at $320 \mathrm{~nm}$ for 24 seconds. Exposure times would vary depending on the thickness of the resist.

Post exposure bake is performed for 5 minutes at $65^{\circ} \mathrm{C}$ and 10 minutes at $95^{\circ} \mathrm{C}$ on a hot plate, then it is left for cooling for 5 minutes.

Photo resist is developed by immersing the sample for 3 minutes in SU 8 developer solution.

Subsequent rinsing is performed in iso-propanol or acetone to remove the residues of the developer. The thickness of the channels is 40 microns with this process. 

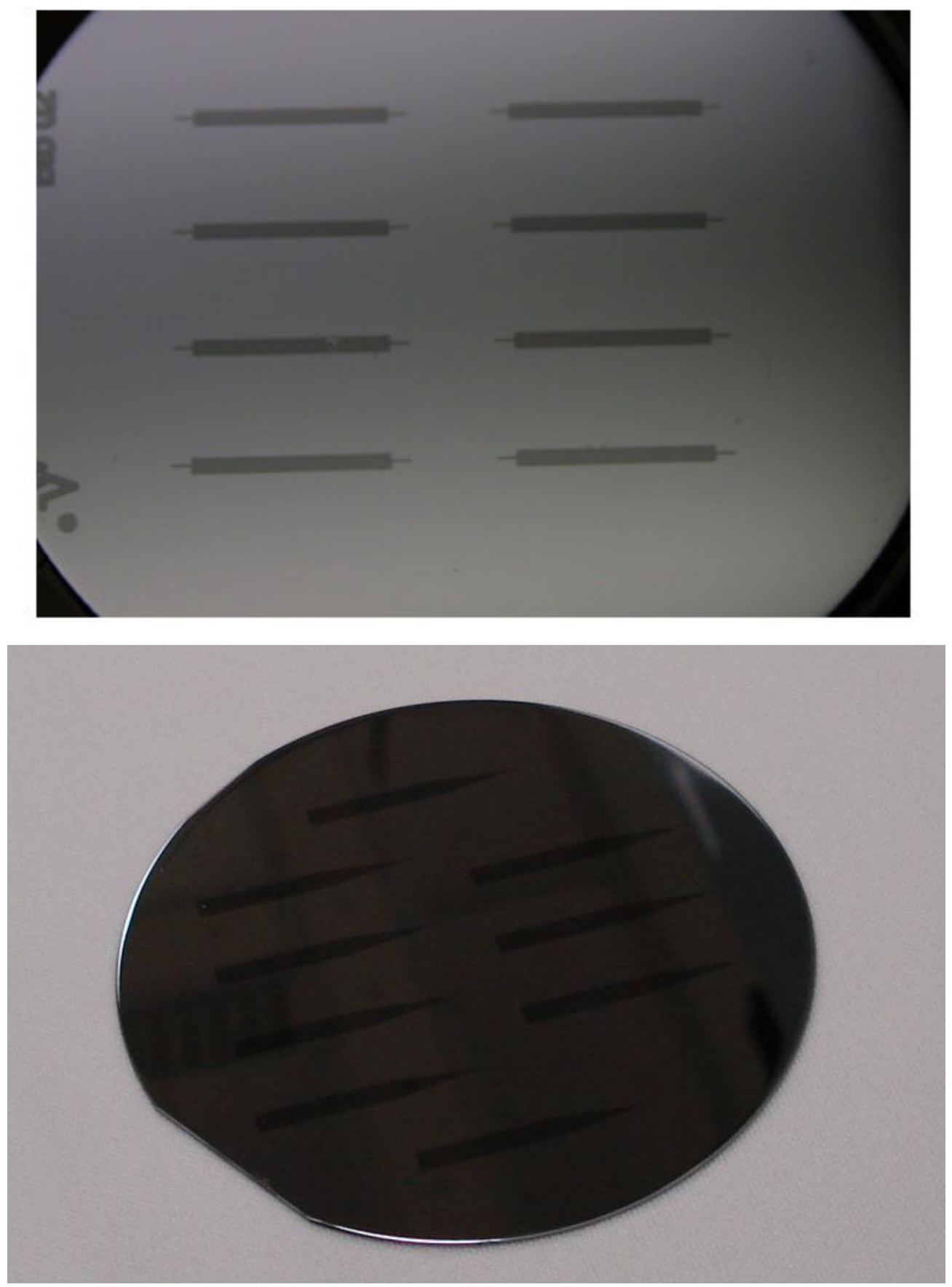

Figure 3.11 Silicon master fabricated using Nano SU8-25.

PDMS is preferred as it is inexpensive, easily molded from the prepolymer, and has good electrical resistivity, optical transmission properties and thermal conductivity. There are also some unfavorable circumstances associated with using PDMS due to electro osmotic flow and due to its hydrophobicity (difficult to wet the PDMS channels), so hydrophobic analytes get adsorbed on the PDMS channels. 
A PDMS chip containing microfluidic channels was fabricated using a Sylgard ${ }^{\circledR}$ 184 Silicone Elastomer Kit. Elastomer base and curing agent was mixed at a weight ratio of 10:1 by stirring for 40-60 minutes. This mixture has been degassed for $40-60$ minutes with a vacuum pump to remove air bubbles generated during stirring action. It was then applied on the surface of silicon master and cured overnight in the oven at $70-90^{\circ} \mathrm{C}$. the apparatus for making the chip is shown in the fig 3.10 [Minsoo reference]

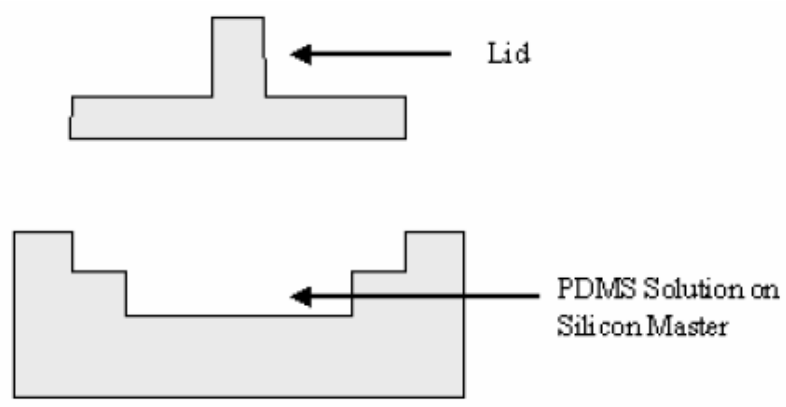

Figure 3.12 PDMS chip making apparatus

The coating layer can be used to vary the surface properties of the PDMS. A solgel process is used to form nanometer sized silica particles throughout polymerized PDMS surface.

Tetraethyl orthosilicate (TEOS) is hydrolyzed and then condensed using an acid or base on the PDMS surface [34]. TEOS solution (volumetric ratio of 2:1:1:1) with TEOS, ethanol, DI water and 5mM HCL is made and this solution is made to flow continuously for 30 60 minutes to coat the PDMS with permanent free silanol groups and thus would increase Electro osmotic flow and wettability. This reduces the absorption of the hydrophobic materials and would allow sealing of PDMS to another PDMS sample if necessary.

\subsubsection{PDMS binding to the Alumina Surface}

There are many techniques to bind glass to glass and silica to silica using PDMS but no established technique, to our knowledge, is present for binding PDMS to alumina surface. Oxygen Plasma binding is adopted to bind the PDMS chip to the alumina surface as binding glass to PDMS and getting irreversible seals is widely known. 
It is very important for the PDMS to stick to the alumina surface, if the PDMS is not properly bonded to the alumina surface then this would result in high scattering loss at the time of measurements. The changes in surface chemistry have been well studied by different research groups. The research concludes that PDMS material in general has chains of $-\mathrm{O}-\mathrm{Si}\left(\mathrm{CH}_{3}\right)_{2}-$, which on exposure of oxygen plasma develops silanol groups ($\mathrm{OH})$ at the place of methyl groups $\left(-\mathrm{CH}_{3}\right)$ [36]. The oxidation of the surface layer (in this case alumina or glass) increases the hydroxyl groups and this leads to the formation of strong intermolecular bonds. The silanol groups condense with those of a similar surface when brought into contact. The PDMS to glass surface produce Si-O-Si bonds while we expect an Al-O-Si bond between the PDMS and alumina interface. This covalent bond would form the basis for the tight irreversible seal between the layers. The process for PDMS binding to Alumina Surface is given below:

1. Clean the alumina substrate using a series of polar and non polar solvents consecutively 5 min each.

2. Bake the Alumina sample to dehydrate the surface for 10 minutes at $100{ }^{\circ} \mathrm{C}$.

3. Sonicate the PDMS chip in Isopropanol for 30min to remove the surface impurities if any.

4. Bake the PDMS chip in oven for $15 \mathrm{~min}$ at $100 \mathrm{C}$

5. Place the samples in the plasma cleaner chamber taking care that the surfaces to be bonded are faced to the plasma.

6. Expose the samples for a span of minute.

7. Hydrolyze the surfaces (formation of $\mathrm{OH}$ bonds) by dipping them in de-ionized water for $10 \mathrm{~min}$.

8. Align the sample (Alumina and PDMS) and bake it for eight hours or more at $100 \mathrm{C}$. 


\section{EXPERIMENTAL RESULTS}

\subsection{Fabrication of Alumina waveguides}

The design of the SPARROW biosensor needs a high contrast in the refractive indices of waveguides and spacer layers, so in an attempt to grow alumina waveguides with desired indices we performed a series of depositions. It was found that the losses and refractive index of the films deposited were a function of chosen growth parameters.

\subsubsection{Refractive index function of Ion source parameters \& Optical Propagation Loss function of Ion source parameters}

Our group worked on optimization of silica growth. Single films of alumina were explored. The $\mathrm{Al}_{2} \mathrm{O}_{3}$ source material used was $99.99 \%$ pure pieces of size $2-12 \mathrm{~mm}$. By controlling and varying ion source operation parameters, we found that the refractive index of $\mathrm{Al}_{2} \mathrm{O}_{3}$ could be engineered within a range of approximately 1.58 to 1.64 .

Inlet oxygen gas flow rate and ion source drive current are the two main cold cathode ion source parameters that influenced the properties of alumina films. Figure 4.1 is the plot showing the dependence of index of refraction with oxygen flow rate and ion source drive current. The error bars indicate an index uncertainty of $+/-0.001$ returned from the ellipsometer, which was determined from the mean square error result returned when attempting to fit the model parameter data to data obtained experimentally [31].

All the films deposited had light guiding capability that varied according to the ion beam parameters used for deposition. The propagation losses for these films were calculated using a prism to couple light from a He-Ne laser of wavelength $633 \mathrm{~nm}$ into the waveguides. The waveguide streak is imaged using a CCD camera and the losses are calculated by processing the image of the light scattered from the surface of the film as described in Chapter 3 and Appendix B.

In a cold cathode source, gas flow rate and ion source drive current are the two parameters that control the flux of ions and energy delivered to the depositing film. The ion source drive voltage is the parameter most related to the mean ion energy, which is related as: $E_{\text {mean }} \sim 0.5 e V_{d}$, where $V_{d}$ is the ion source drive voltage. The increasing drive current versus drive voltage relationship experimentally observed indicates that with other parameters held constant, increasing the drive current increases the mean ion 
energy for emitted ions. Conversely, when oxygen flow rate is increased, the chamber pressure rises higher and the ion source must lower its drive voltage and thus, mean ion energy should be lower [31, 32].

The ion energy distribution functions (IEDF) for cold cathode ion sources running with oxygen are presented in [31] and shows that the ion current density increases with increase in drive current thereby increasing the mean ion energy. The deposited material densification observed through the increasing index of refraction for increasing drive currents with higher ion flux is shown in Figure 4.1. It can also be seen from the distribution in [31] that increasing the $\mathrm{O}_{2}$ flow decreases both the mean ion energy and ion current density. It was observed in our experiments that increasing $\mathrm{O}_{2}$ flow increases chamber pressure and lowers drive voltage. Due to this low drive voltage we expect lower material density and a corresponding lower index of refraction as shown in Figure 4.1 .

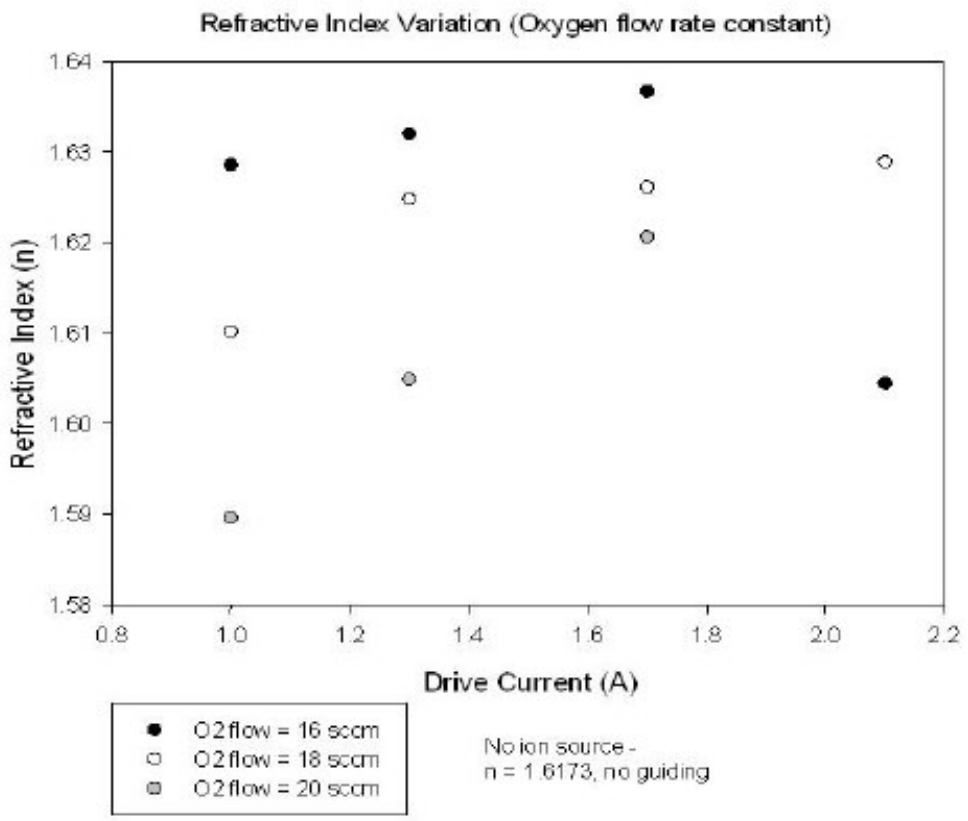

Figure 4.1 Variation of propagation loss for various oxygen flow rates with ion source drive current fixed

For the above study in which we grew films with varying ion source drive currents and inlet oxygen flow rates, the optical quality was assessed in every case. Optical film quality refers to the scattered streak quality and optical propagation loss. We obtained a relation that the optical propagation loss increased with ion source drive 
current is shown in Figure.4.2. The losses for these films were calculated using the optical scattering measuring technique [30] as detailed in chapter 3 and appendix B.

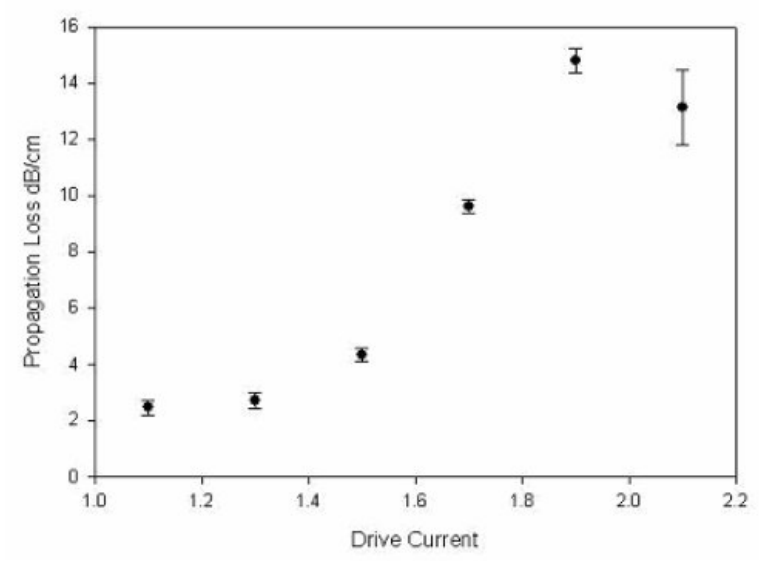

Figure 4.2 Optical propagation loss as a function of Ion source Drive current

The optical propagation loss for a film grown with $1.3 \mathrm{~A}$ is $1.69+/-0.5 \mathrm{~dB} / \mathrm{cm}$ and for $2.1 \mathrm{~A}$ is $5.38+/-1.15 \mathrm{~dB} / \mathrm{cm}$ for $16 \mathrm{sccm}$ of oxygen flow rate.

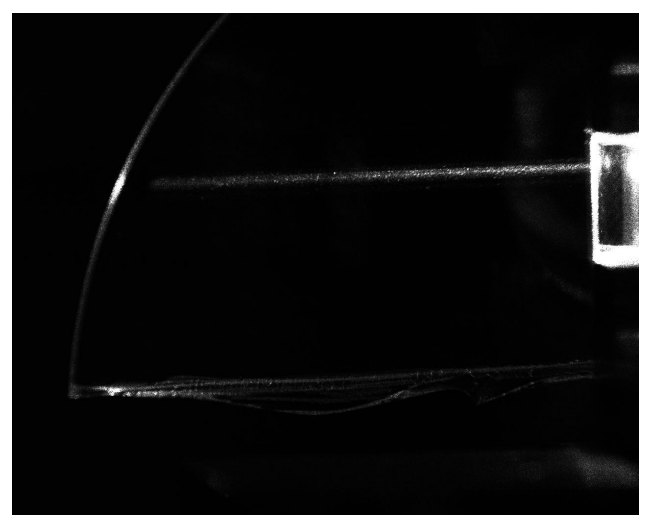

Figure4.3a B561A low loss film

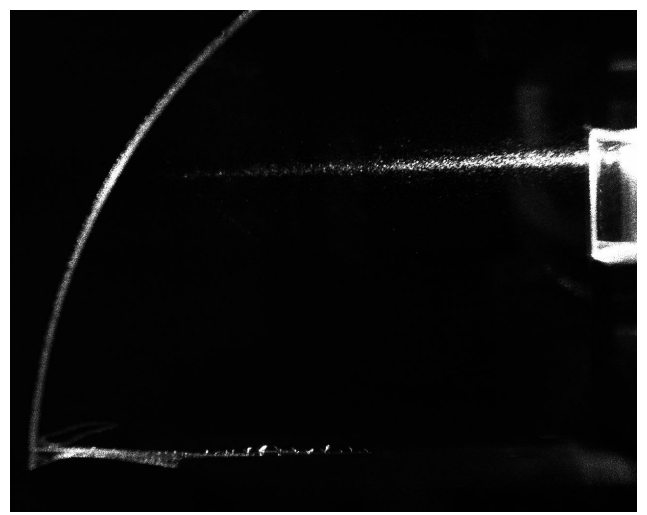

Figure 4.3 b B548 high loss film 


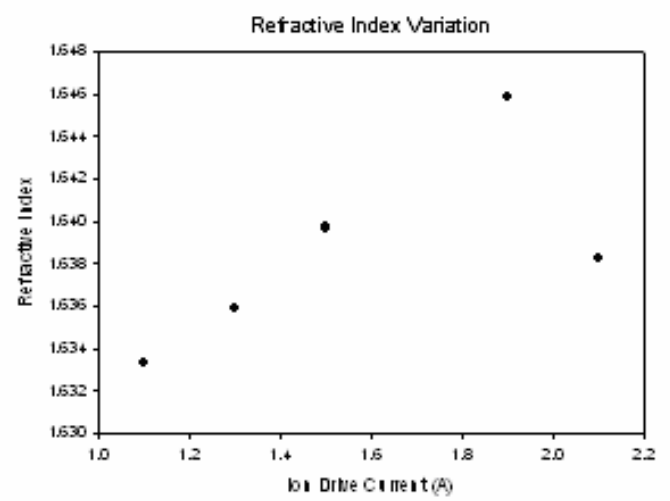

Figure 4.4 Refractive index as a function of Ion source Drive current

\begin{tabular}{|c|c|c|c|c|c|c|c|c|}
\hline $\begin{array}{c}\text { Sample } \\
\#\end{array}$ & $\begin{array}{c}\text { O2 flow } \\
(\mathrm{sccm})\end{array}$ & $\begin{array}{c}\text { Drive Current, } \\
\mathrm{Id}(\mathrm{A})\end{array}$ & $\begin{array}{c}\text { Drive Voltage } \\
\mathrm{Vd}(\mathrm{V})\end{array}$ & $\mathrm{n}$ & $\mathrm{k}$ & Thickness & $\begin{array}{c}\text { Loss } \\
(\mathrm{dB} / \mathrm{cm})\end{array}$ & $\begin{array}{c}\text { Loss } \\
\text { error }\end{array}$ \\
\hline B548A & 18 & 1.3 & 157 & 1.6271 & 0 & 176.54 & 4.79 & 0.729 \\
\hline B550A & 18 & 1.5 & 165 & 1.6322 & 0.00071 & 175.644 & 4.84 & 0.87 \\
\hline B549A & 18 & 1.7 & 161 & 1.6384 & 0.0048 & 166.795 & 3.04 & 0.387 \\
\hline B553A & 18 & 2.1 & 178 & 1.6386 & 0 & 164.385 & 3.76 & 0.677 \\
\hline B554A & 18 & 1.9 & 160 & 1.6488 & 0.000648 & 163.17 & 4.858 & 0.783 \\
\hline
\end{tabular}

Table 4.1 shows different sample grown under different drive currents with fixed oxygen flow rate

\subsubsection{Surface Characterization}

Surface roughness was measured for both these films using ellipsometer and the images are shown in Figure 4.5a and Figure 4.5 b.
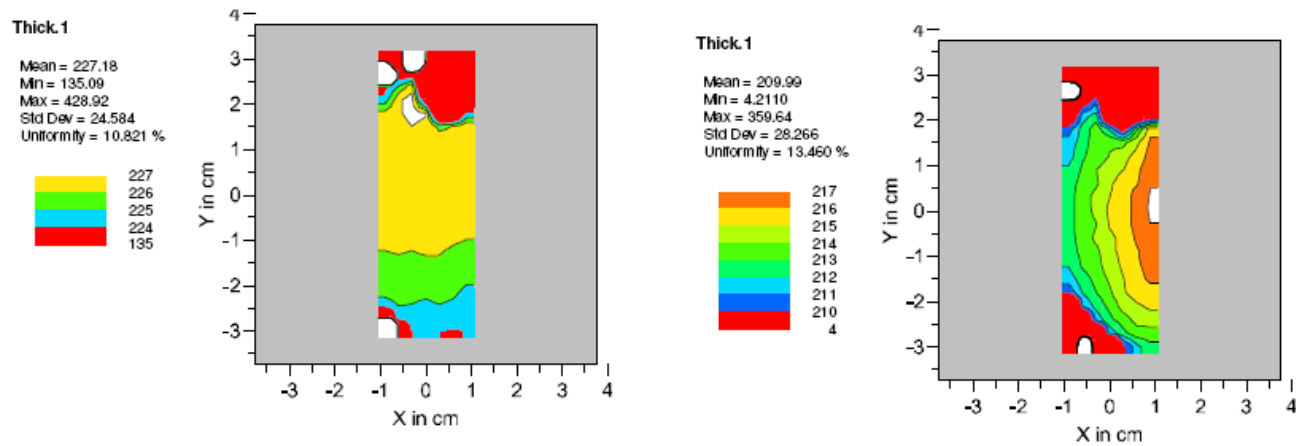

Figure 4.5 Surface characterization done on two different films with low loss and high loss film

The inlet oxygen gas flow rate also had a higher influence on the optical quality of the films. If the oxygen flow rate is high inside the chamber then there is high flux generated. This results in an increase in the amount of gas molecules present in the chamber and thus a decrease in the mean free path. The propagation loss for films with 
different oxygen flow rate is shown in Figure 4.6 Conversely, with lower oxygen flow rates there are insufficient gas molecules to create or to maintain the plasma.

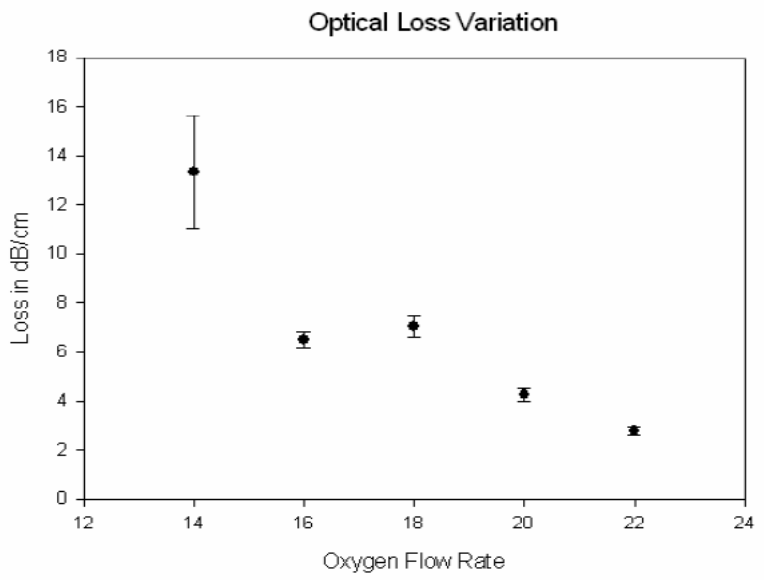

Figure 4.6 Optical propagation loss as a function of oxygen flow rate

Single layer alumina films grown for a set of ion source drive currents and oxygen flow rates and the relationship between these parameters and the index of refraction is shown in Figure 4.1. From this study we chose an intermediate value of ion source drive current of $1.3 \mathrm{~A}$ and oxygen flow rate of $16 \mathrm{sccm}$ for depositing alumina waveguides for our SPARROW sensor.

Surface roughness was also measured using AFM. The deposited films showed low rms roughness. Decreasing the surface roughness to the minimum value possible would provide the best conditions for the biolayer to grow.
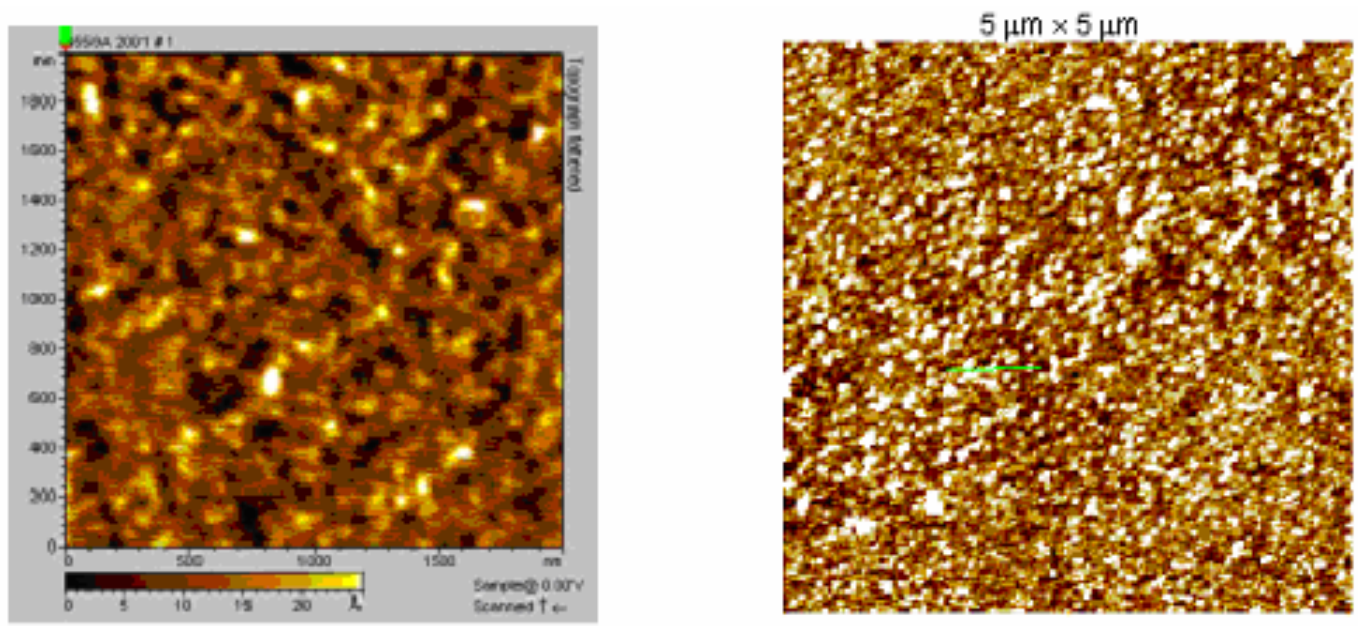

Figure 4.7 AFM image left (2000 nm x $2000 \mathrm{~nm}$ ) RMS roughness of $0.28 \mathrm{~nm}$, right (5000 nm x $5000 \mathrm{~nm})$ RMS roughness of $0.48 \mathrm{~nm}$ of bare alumina surface

\section{SEM and EDS results}




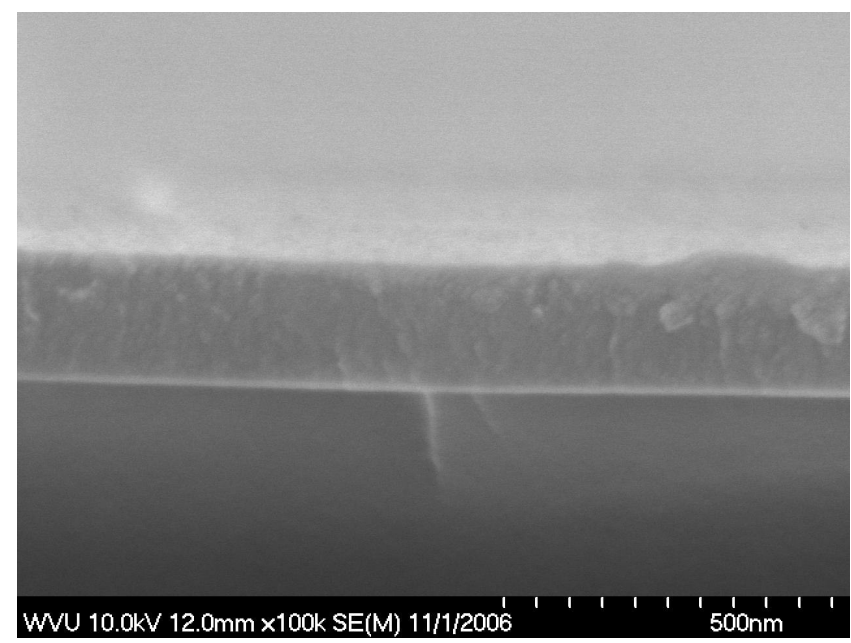

Figure 4.8 SEM image of a single alumina layer (sample B540A)

From the EDS results we found that there could be contamination after metal depositions for which the e-beam system is used, such as Platinum in Figure 4.9 in a single layer alumina deposition. To avoid contamination from other metal depositions the shields inside the chamber were cleaned and the films were tested with SEM and EDS.

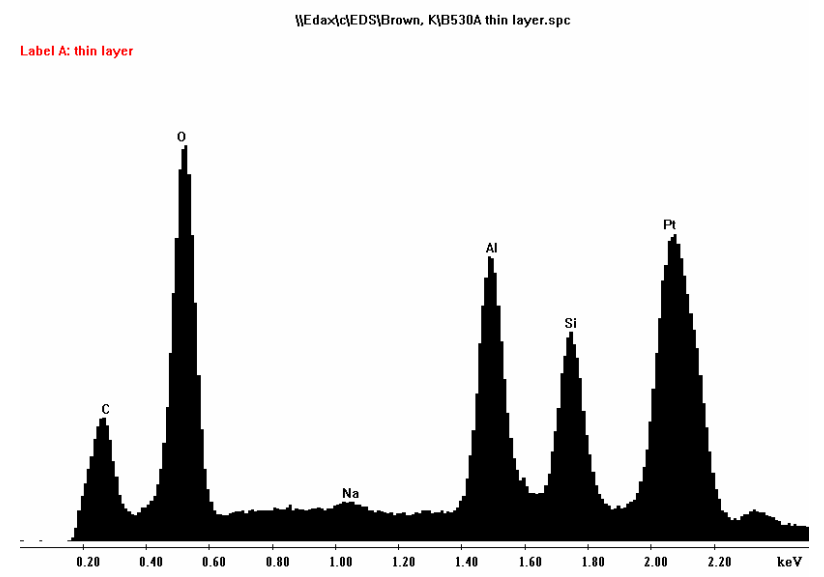

Figure 4.9 EDS for the above sample to check for contamination

\subsection{Beta Values}

With these optimized growth processes for the alumina waveguides, stacks were grown. After the growth the structure is taken out of the chamber and the beta values are measured and compared to the simulated values for verification purpose. The thickness and index of refraction obtained from ellipsometer can be indirectly verified by measuring the propagation constants of the waves as the modal angle depends on these parameters. 


\section{Comparison of simulated and measured beta values}

When a plane wave travels in a waveguide it interferes as it propagates to form a repeating pattern. The propagation constant or the beta value of a plane wave is the periodic frequency at which the wave repeats and it is given as

$$
\beta=k n_{\text {eff }}=\frac{2 \pi}{\lambda} n_{\text {eff }}
$$

The propagation constant depends on the modal angle of the waveguide, refractive index of the waveguide and the wavelength of propagating light.

The beta values can be calculated from the '2D Modesolver' by using the ellipsometry data and these values are compared to the beta values obtained from the prism coupler measurements. Comparison of beta values obtained from simulation and prism coupler for a few samples are shown in Table 4.2a and 4.2b.

\begin{tabular}{|c|c|c|}
\hline Sample & Beta0(Simulated) & $\begin{array}{c}\text { Beta0(Prism } \\
\text { Coupler) }\end{array}$ \\
\hline B422A & 14.78797 & 14.7511 \\
\hline B428A & 14.783 & 14.9281 \\
\hline B437A & 14.8436 & 14.8743 \\
\hline
\end{tabular}

Table 4.2a Beta values for single layer alumina films

\begin{tabular}{|c|c|c|c|c|}
\hline Sample & $\begin{array}{c}\text { Beta0 } \\
\text { (Simulated) }\end{array}$ & $\begin{array}{c}\text { Beta1 } \\
\text { (Simulated) }\end{array}$ & $\begin{array}{c}\text { Beta0 (Prism } \\
\text { Coupler) }\end{array}$ & $\begin{array}{c}\text { Beta1 (Prism } \\
\text { Coupler) }\end{array}$ \\
\hline B502AOA & 14.97646 & 14.7836 & 14.9729 & 14.77604 \\
\hline B503AOA & 14.97687 & 14.84931 & 14.99676 & 14.82376 \\
\hline B504AOA & 15.02758 & 14.9036 & 15.03653 & 14.85856 \\
\hline
\end{tabular}

Table 4.2b Beta values for structures with two modes

Table 4.2a shows the beta values of single layer alumina films deposited on borofloat substrates and Table $4.2 \mathrm{~b}$ shows the beta values of structures with aluminasilica-alumina layers grown on a borofloat substrate.

\subsection{Issues with Ellipsometer Measurement/Modeling}

Proper modeling using the ellipsometer could not be achieved for the stacked waveguides. It was experimentally found that ellipsometry modeling for all the samples was incorrect. The experimentally observed $n_{\text {eff }}$ from the prism coupler was not matching with the beta values obtained from the simulations when ellipsometry data was given as input for the simulation tool. Since the ellipsometer gave correct models for single layer films, single layers of alumina and silicon dioxide were deposited (and the tooling factor 
was corrected) and the $\mathrm{n}_{\mathrm{eff}}$ or modal index were measured using the prism coupler which matched the $\mathrm{n}_{\mathrm{eff}}$ from the simulations and also the design.

From the data obtained from these single layers a model was designed by fixing the indices and thicknesses. Now after deposition we had beta value data from three different sources. Errors were calculated from which it was inferred that the ellipsometry modeling was incorrect and our assumption of single layer data was more accurate and consistent and matched the beta values of the prism coupler.

\begin{tabular}{|c|c|c|}
\hline \multicolumn{3}{|c|}{ Top waveguide } \\
\hline $\begin{array}{c}\text { Measured } \\
\text { Neff (PC) }\end{array}$ & $\begin{array}{c}\text { Ellipsometry } \\
\text { Data Neff }\end{array}$ & $\begin{array}{c}\text { Fixed } \\
\text { Neff }\end{array}$ \\
\hline 1.5066 & 1.516 & 1.51 \\
\hline 1.5084 & 1.5205 & 1.511 \\
\hline 1.508 & 1.5235 & 1.512 \\
\hline 1.51 & 1.5056 & 1.51 \\
\hline
\end{tabular}

\begin{tabular}{|c|c|c|}
\hline \multicolumn{3}{|c|}{ Bottom waveguide } \\
\hline $\begin{array}{c}\text { Measured } \\
\text { Neff (PC) }\end{array}$ & $\begin{array}{c}\text { Ellipsometry } \\
\text { Data Neff }\end{array}$ & $\begin{array}{c}\text { Fixed } \\
\text { Neff }\end{array}$ \\
\hline 1.5009 & 1.506 & 1.4868 \\
\hline 1.5018 & 1.5145 & 1.5064 \\
\hline 1.5022 & 1.5149 & 1.5087 \\
\hline 1.4897 & 1.5005 & 1.4974 \\
\hline
\end{tabular}

Table 4.3 shows the data of top and bottom waveguide in a stack

Top waveguide

- $\%$ Error average for Measured vs Fixed : 0.001

- \%Error average for Measured vs Ellipsometry : 0.0024

Bottom Waveguide

- $\%$ Error average for Measured vs Fixed : 0.0056

- \%Error average for Measured vs Ellipsometry: 0.0061

The above tables show the $\mathrm{n}_{\mathrm{eff}}$ values for samples where "measured" refers to the actual observed value from the Prism coupler, the second column shows the Neff calculated from the ellipsometry data and the last column shows the $n_{\text {eff }}$ values for the model that were obtained by fixing the thickness and indices from the single layer films data.

From the errors calculated from these values we infer that fixing the thicknesses and indices gives less error to the measured $\mathrm{n}_{\mathrm{eff}}$ values than the ellipsometry data.

\subsection{Flow Cell Experiment}

Figure 4.10 shows the layout of the experimental setup used for performing the flow cell experiments. 


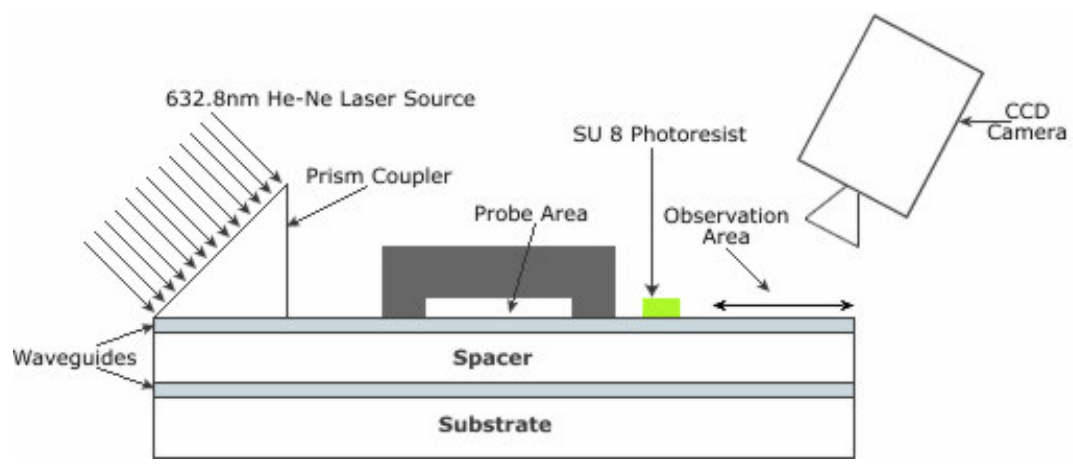

Figure 4.10 shows the complete experimental setup

Figure 4.11a shows the PDMS micro fluidic channel with input and output capillary tubes setup used for flow cell experiments. Figure $4.11 \mathrm{~b}$ shows the experimental setup used for performing the flow cell experiment. The PDMS chip is bonded onto the top alumina waveguide using a oxygen plasma-aided bonding method as explained in chapter 3. Inlet and outlet holes were drilled using a driller and capillary tubes were used for injecting liquid with an aid of a syringe pump. Syringe pump can inject fluids at very slow flow rates and thus pressure in the channel can be controlled. It can be seen in the layout that there is a thin strip of SU8 photoresist applied after the channel, this application of photoresist was done to differentiate the two waveguides, since the SU8 has higher index of about 1.67 when light is coupled into the top waveguide the light is completely decoupled from the top waveguide. Earlier the top waveguide has been etched to differentiate the two waveguides, etching the sample gave unwanted reflections on the borofloat which interfered with the actual streak in the observation area and caused high scattering losses. A Quantum Imaging Retiga 1300 cooled digital CCD camera is used for power measurements. A Harward Apparatus microprocessor-based syringe pump was used for pumping fluids into the channel. 


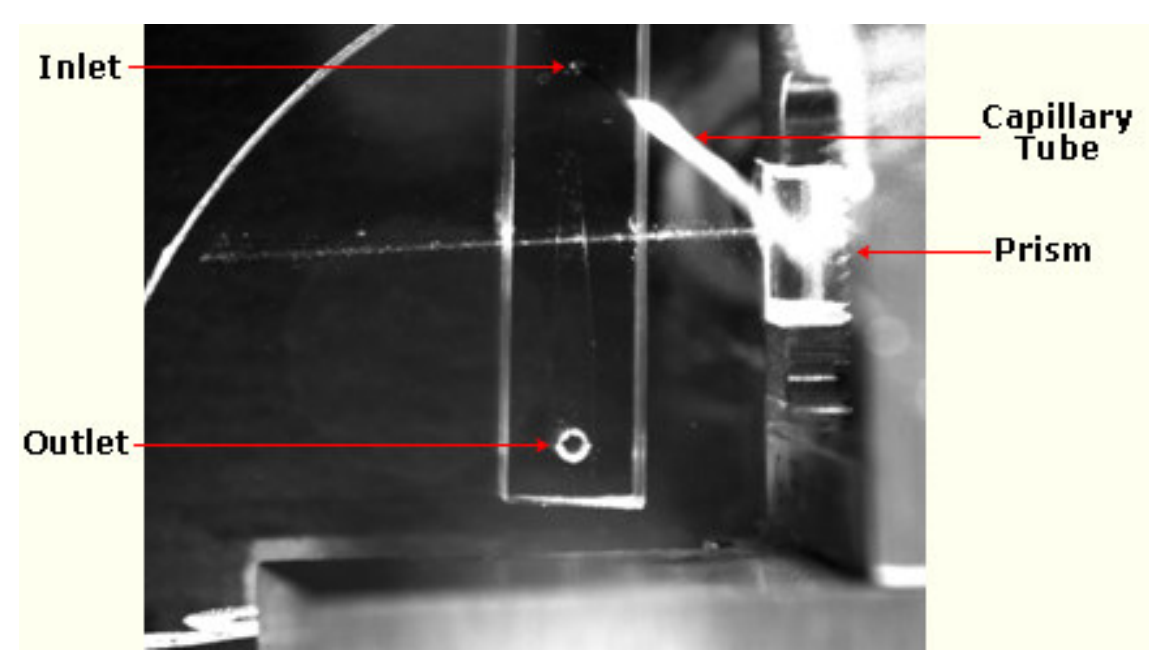

Figure 4.11a shows observation area with light coupled into waveguides

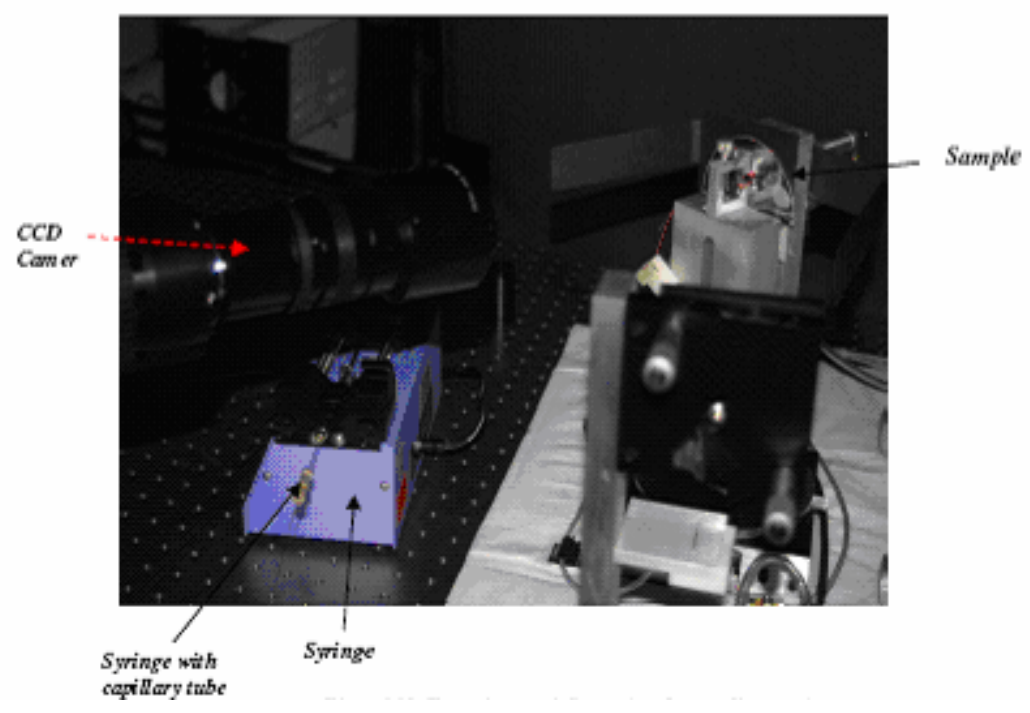

Figure $4.11 \mathrm{~b}$ shows the experimental setup in the Laser lab (WVU)

\subsubsection{Experimental Procedure}

As in indicated in Chapter 3, we need to know the operation limits of the device. The first of them is the refractive index of the liquid flowed in the channel. The Biolayer is constituted of SAMs (self assembled monolayer), Streptavidin, Biotin and Fluorescent beads conjugate. The effective refractive index of the whole bio-layer would be in the range of $1.4-1.5$. The refractive index of DI water is 1.3318 , so the effective refractive index of the whole bio-layer with water is expected to vary from $1.3318-1.45$ depending on the percentage composition of the bio-layer, water and also the percentage surface area covered by bio-layer. We have chosen sucrose solution, with different 
concentrations of sucrose and the indices for these solutions range from $1.3318-1.4$ [41] based on the mass fraction of glycerol and is near to the operation range of the device. The refractive index of PDMS chip is in the range of $1.41-1.45$, and there should be no power transfer at this index range. Effectively the device should be tuned in the range of 1.33- 1.39 for device operation.

Figure 4.12 shows a sample structure B630AOA with 'n' as refractive index and ' $d$ ' is the thickness of each layer in the structure.

\begin{tabular}{|l|}
\hline Alumina $\mathrm{n}=1.616 \quad \mathrm{~d}=250 \mathrm{~nm}$ \\
\hline Silica $\mathrm{n}=1.47 \quad \mathrm{~d}=1006 \mathrm{~nm}$ \\
\hline Alumina $\mathrm{n}=1.616 \mathrm{~d}=176 \mathrm{~nm}$ \\
\hline Borofloat $\mathrm{n}=1.4822 \mathrm{~d}=1000 \mathrm{~nm}$ \\
\hline
\end{tabular}

Figure 4.12 B630AOA sample structure

The electric field distribution with air as cladding layer is shown in Figure 4.13a, the total power is present in one waveguide and Figure 4.13b shows the electric field distribution for water as cladding layer where there is power transfer between the waveguides and is tuned for water.

Optical Field

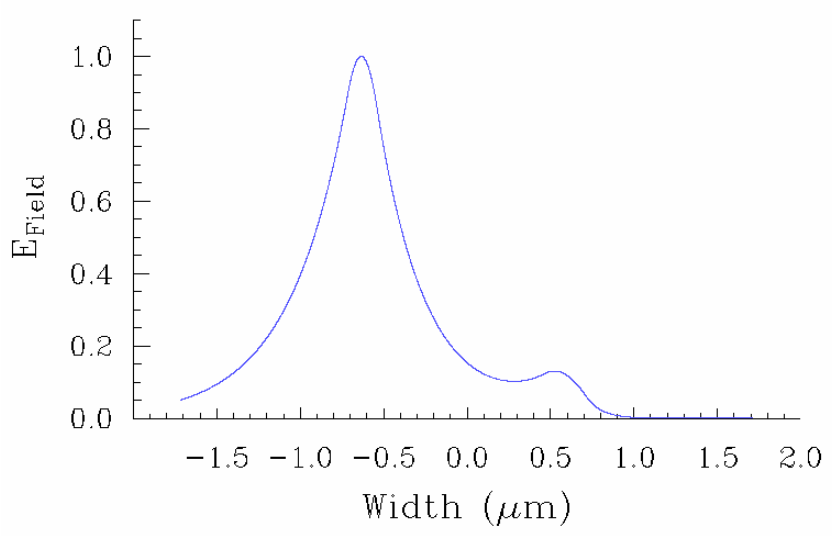


Figure 4.13a Electric field distribution for air as cladding layer

\section{Optical Field}

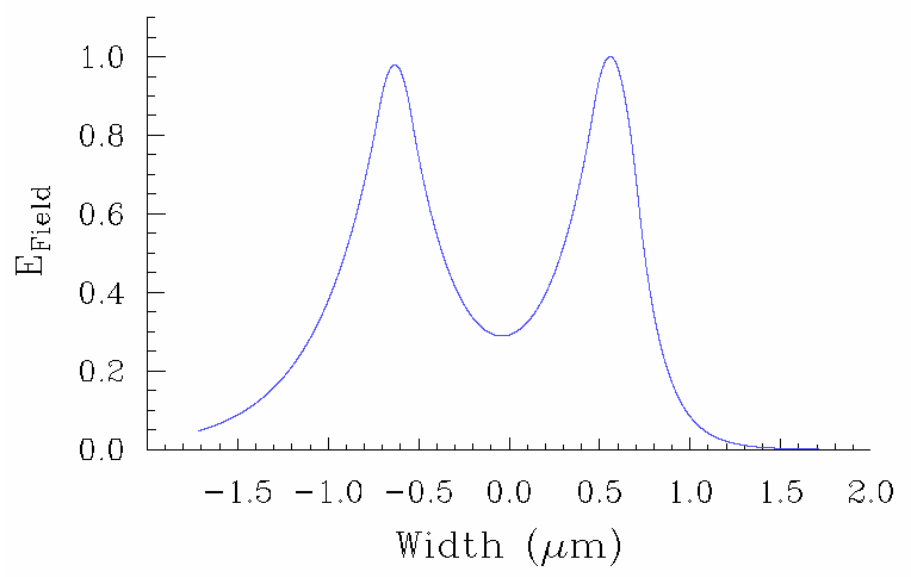

Figure 4.13b Electric field distribution for water as analyte

Light is coupled into the bottom waveguide with no analyte in the channels. An image with light coupled into bottom waveguide is also taken to compute the losses in the bottom waveguide alone. Analyte is pumped into the microfluidic channel with a syringe pump and images of the observation area are taken. Figure 4.10 shows the observation area to be imaged. The loss calculated in the observation area should be the same for all the cases and should be equal to the case when there is no analyte in the solution.

When laser light is coupled into the top waveguide and the power is observed in the observation area. The power was not detected in the observation area as the light streak ended in the top waveguide after passing through the PDMS, channel interaction area and SU8 interfaces. Based on the modeling design from the 2D Mode solver the structure should support total power total power transfer in the channel area but this has not been observed. So light was coupled into the bottom waveguide and the power was observed in the observation area before and after the water flow and changes in the power are measured. 


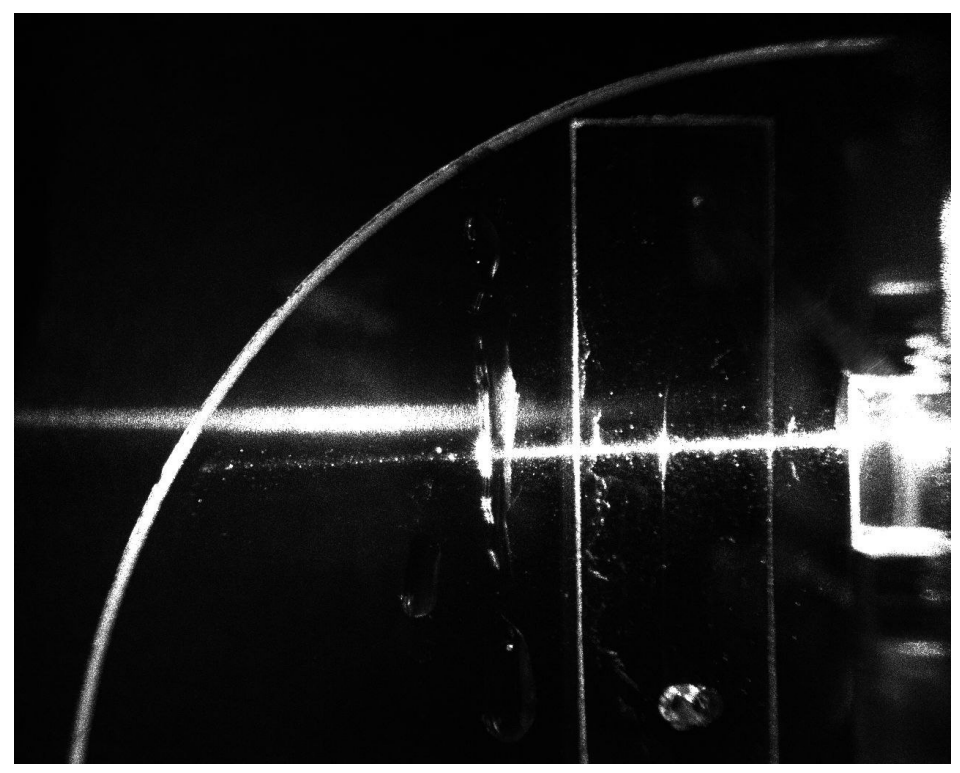

Figure 4.14 shows Sample structure B630AOA with water as analyte

The above Figure 4.14 shows the output obtained when water is flowed through the channel. There is power detected in the observation area.

The procedure for detecting the analyte refractive index is detailed in Appendix $\mathrm{D}$, as image is captured before any analyte is pumped and loss is computed in the observation area using the procedure mentioned in Appendix B. Analyte would be pumped into the channel and the image is to be taken. The detection is based on the intensity of light detected at the ends of the waveguides therefore it is necessary to get maximum data from the observation area. The presence of analyte would reduce the amount of power transfer between the waveguides and light intensity observed in the observation area changes depending on the interaction length and coupling length of the model. Depending on the index of the analyte present in the channel the intensity decreases when compared to water as analyte in the channel. Ideally for analyte solutions of different refractive indices the loss variation should vary and this is shown in Figure 4.15. 


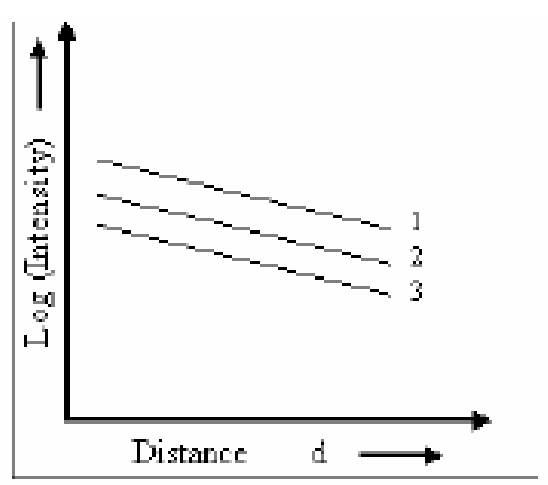

Figure 4.15 Loss variation in the observation area for different analyte solutions

Images are taken using CCD camera. The sum of pixel intensities along columns is computed for both the images that is before and after the analyte flow. The relative changes in the column intensities with and without analyte are compared with the simulation results to detect the analyte [18]. The measurements should be done with extreme care without any change in the physical alignment of the sample during the analyte flow, even the capillary tube position needs to fixed. The region in the observation area being selected should not be large, as the average pixel intensity would be prone to noise.

Figure 4.16 shows the power variation in the waveguides when the index of the analyte is 1.3318 (left) and 1.335 (right) (2\% of sucrose solution) respectively.
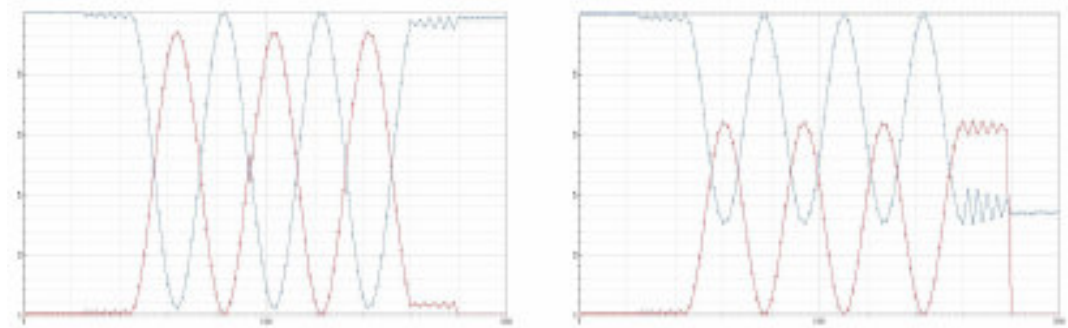

Figure 4.16 power variation in the waveguides

Images of the sample structure taken before and after water flow in the channel. The observation area is marked in red color on the image. Images were taken for different exposure times with water flowing through the channel. Figure 4.17 a \& b are the observation areas for both the cases of before and after the water flow showing the change in the pixel intensities of the region. 


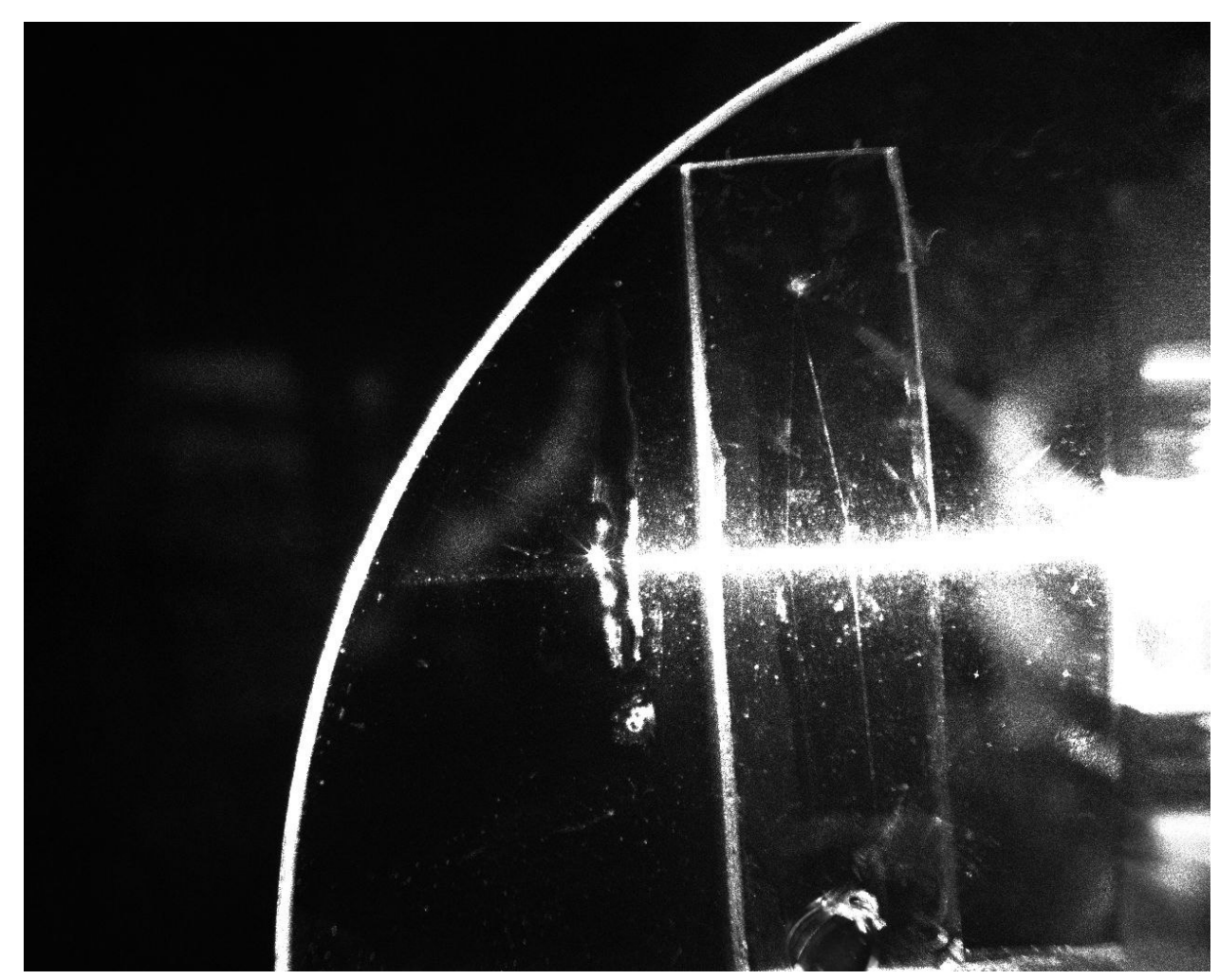

Figure 4.17 a Image when water as analyte

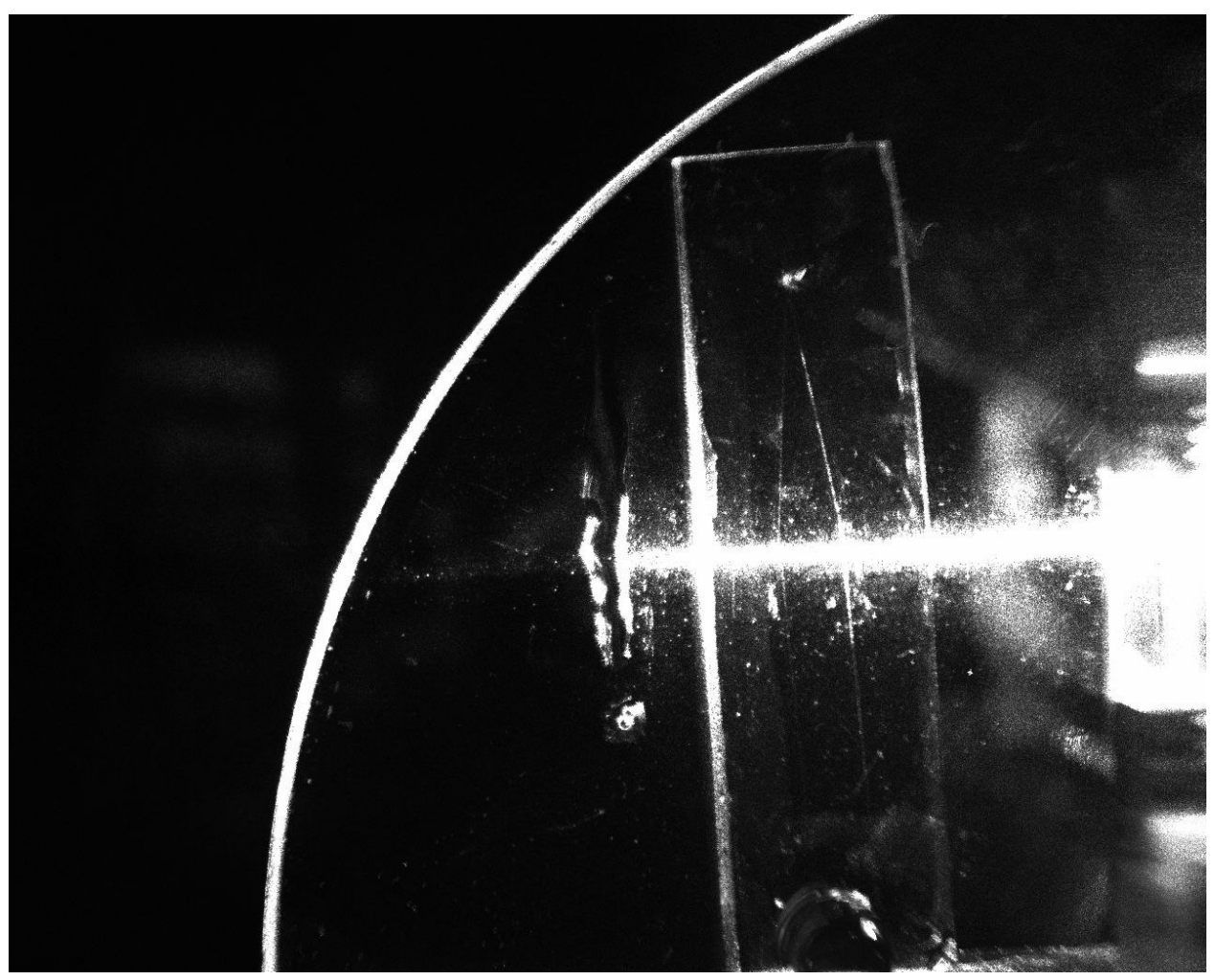

Figure $4.17 \mathrm{~b}$ Image when sucrose as analyte 


\subsubsection{Experimental Protocol for detection of Analyte (sucrose)}

Experimental Procedure

1. First step is to find the refractive indices for different concentrations of sucrose $(1 \%, 5 \%, 10 \%$, and $20 \%)$

2. After PDMS binding to the surface of the stacked waveguide, take the images for loss calculations

3. Place the stack in the prism coupler setup and fix the capillary tube to the inlet of the channel also ensure that the capillary tube is fixed and does not move throughout the experiment (fix the position of the tube).

4. Run DI water through the channel at a speed of $0.9 \mu 1 / \mathrm{min}$, collect the data for different exposure times.

5. Run DI water through the channel with rinse speed for about 10 minutes and collect the data for different exposure times.

6. Repeat step 4 and collect the data again to verify the measurements.

7. Repeat step 5 to verify.

8. Run lowest concentration $(1 \%)$ of sucrose through the channel and take the images for different exposure times.

9. Run DI water through the channel with rinse speed for about 10 minutes and collect the data for different exposure times.

10. Run lowest concentration $(1 \%)$ of sucrose through the channel and take the images for different exposure times.

11. Run DI water through the channel with rinse speed for about 10 minutes and collect the data for different exposure times.

12. Repeat steps 8 through 11 for different concentrations of sucrose.

The above mentioned protocol was followed to perform the flow cell experiment for analytes, DI water, sucrose $(1 \%, 2 \%$ and $5 \%)$ using a stacked waveguide B634AOA. 


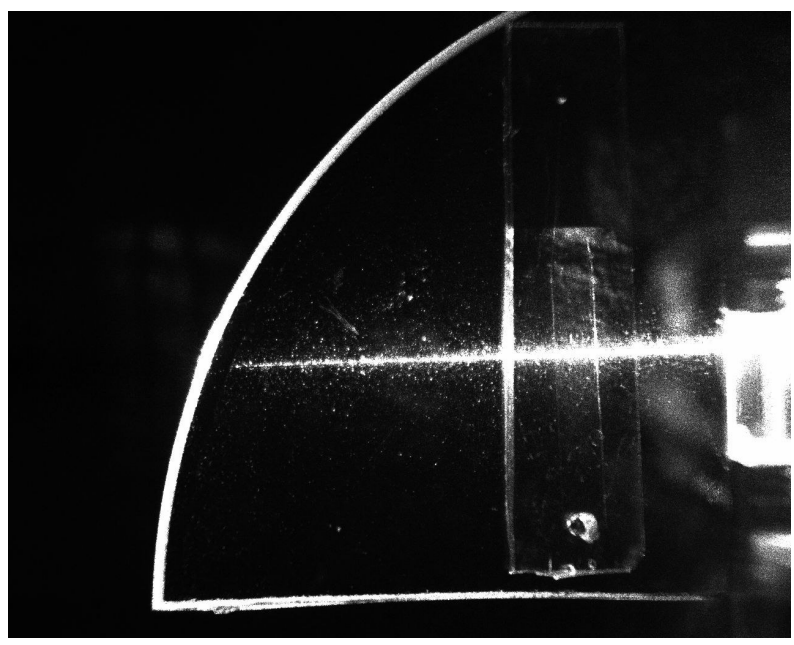

Figure 4.18 B634AOA for exp of 10s with PDMS bottom waveguide

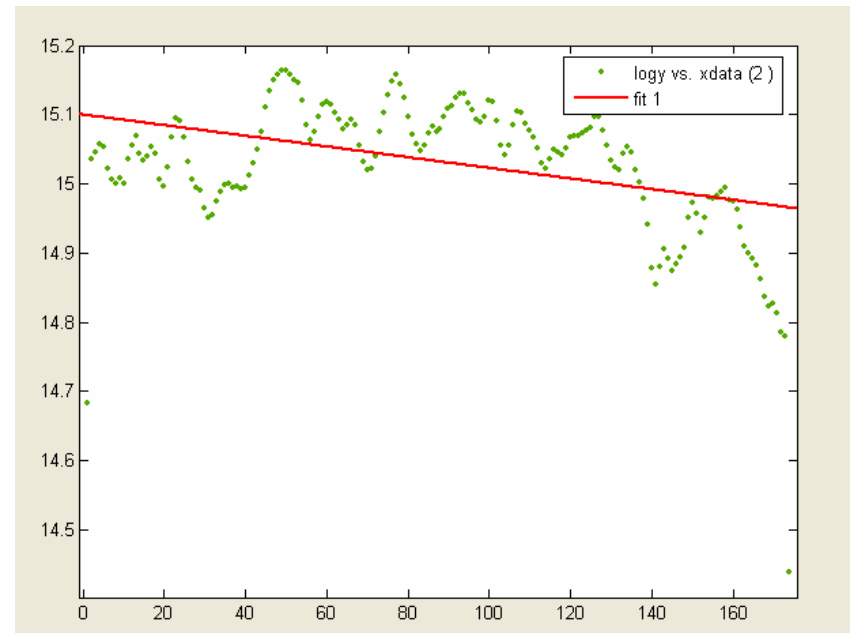

Figure 4.19 Intensity vs pixel distance plot

\begin{tabular}{|c|c|c|c|c|c|c|c|}
\hline $\begin{array}{c}\text { Set Thickness } \\
(\mathbf{n m})\end{array}$ & $\begin{array}{c}\text { Thickness } \\
(\mathbf{n m})\end{array}$ & $\mathbf{R I}$ & $\begin{array}{c}\text { Neff } \\
(\text { Simulated })\end{array}$ & $\begin{array}{c}\text { Thickness } \\
(\mathbf{n m})\end{array}$ & $\mathbf{R I}$ & $\begin{array}{c}\text { Neff Water } \\
(\text { calculations })\end{array}$ & $\begin{array}{c}\text { Neff } \\
(\mathbf{P C})\end{array}$ \\
\hline 250 & 244.96 & 1.6279 & 1.5024 & $\mathbf{2 5 0}$ & $\mathbf{1 . 6 1 6}$ & $\mathbf{1 . 5 1 1 3}$ & $\mathbf{1 . 5 1 5 2}$ \\
\hline 950 & 994.101 & 1.4685 & & $\mathbf{1 0 0 8}$ & $\mathbf{1 . 4 7 4}$ & & \\
\hline 170 & 152.08 & 1.5921 & 1.4946 & $\mathbf{1 7 6}$ & $\mathbf{1 . 6 1 6}$ & $\mathbf{1 . 5 0 8 4}$ & $\mathbf{1 . 4 9 0 3}$ \\
\hline & & 1.4822 & & & & & \\
\hline
\end{tabular}

Table 4.4 Data obtained from Simulations, Ellipsometer and Prism Coupler 


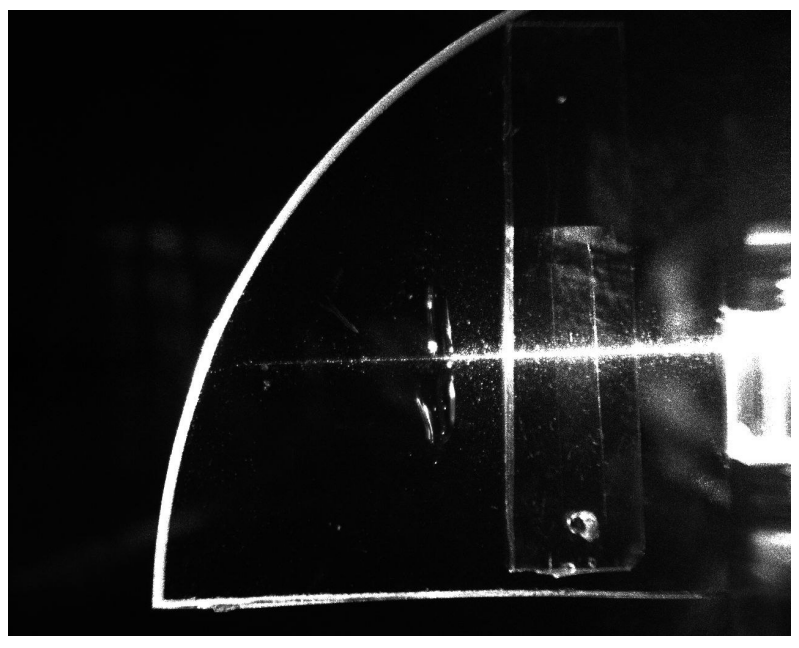

Figure 4.20 B634AOA for exp of 10s with PDMS bottom waveguide with SU 8

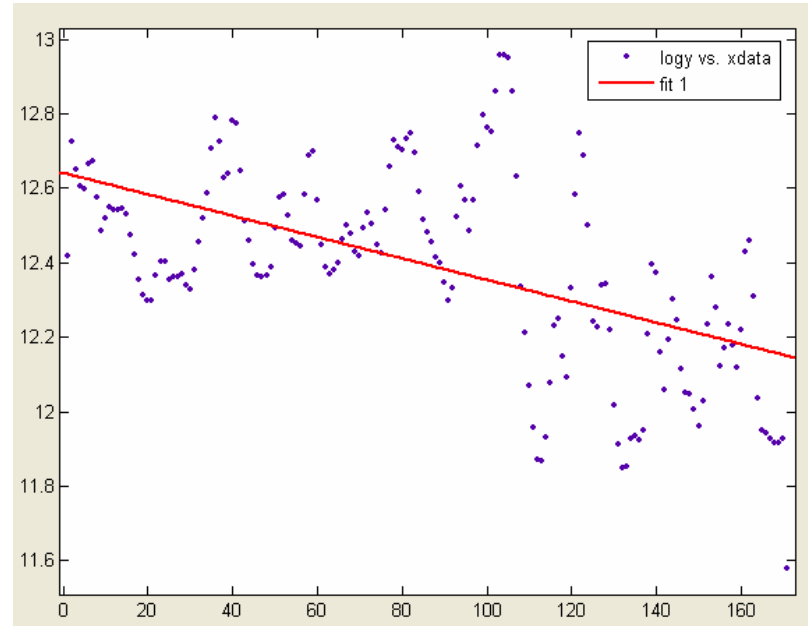

Figure 4.21 Intensity vs pixel distance plot for B634AOA with SU 8

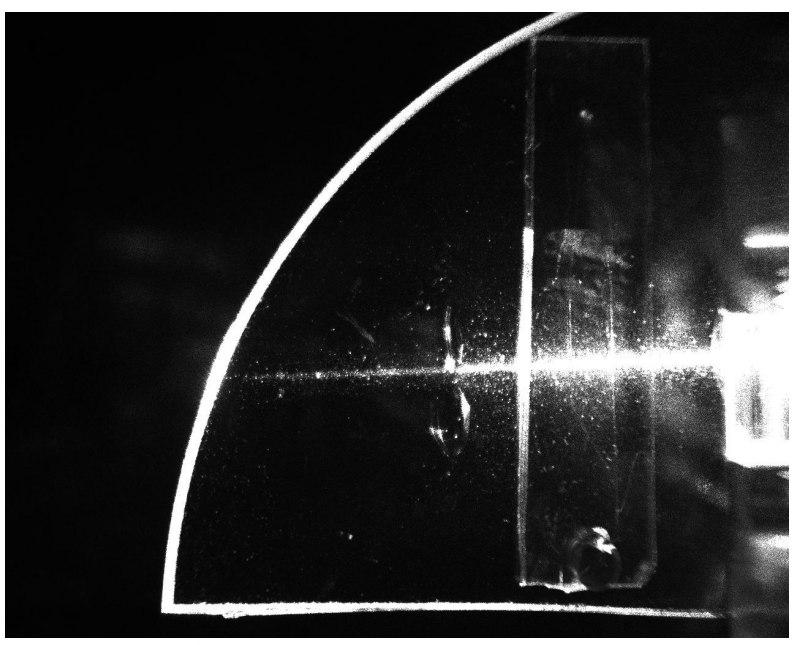

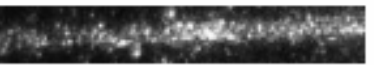

Figure 4.22 B634AOA for exp of 10s with PDMS bottom waveguide with water in the channel 


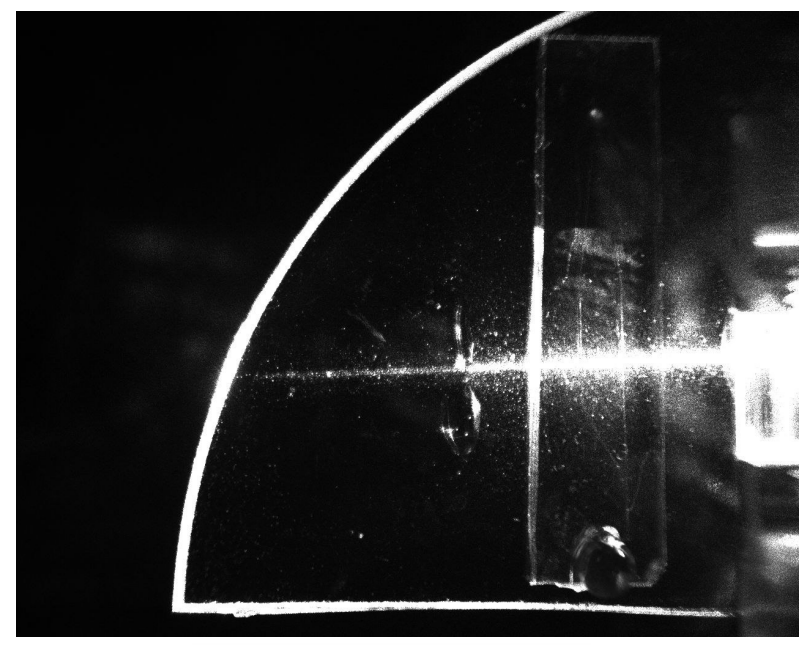

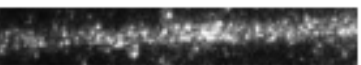

Figure 4.23 B634AOA for exp of 10s with PDMS bottom waveguide with water rinse
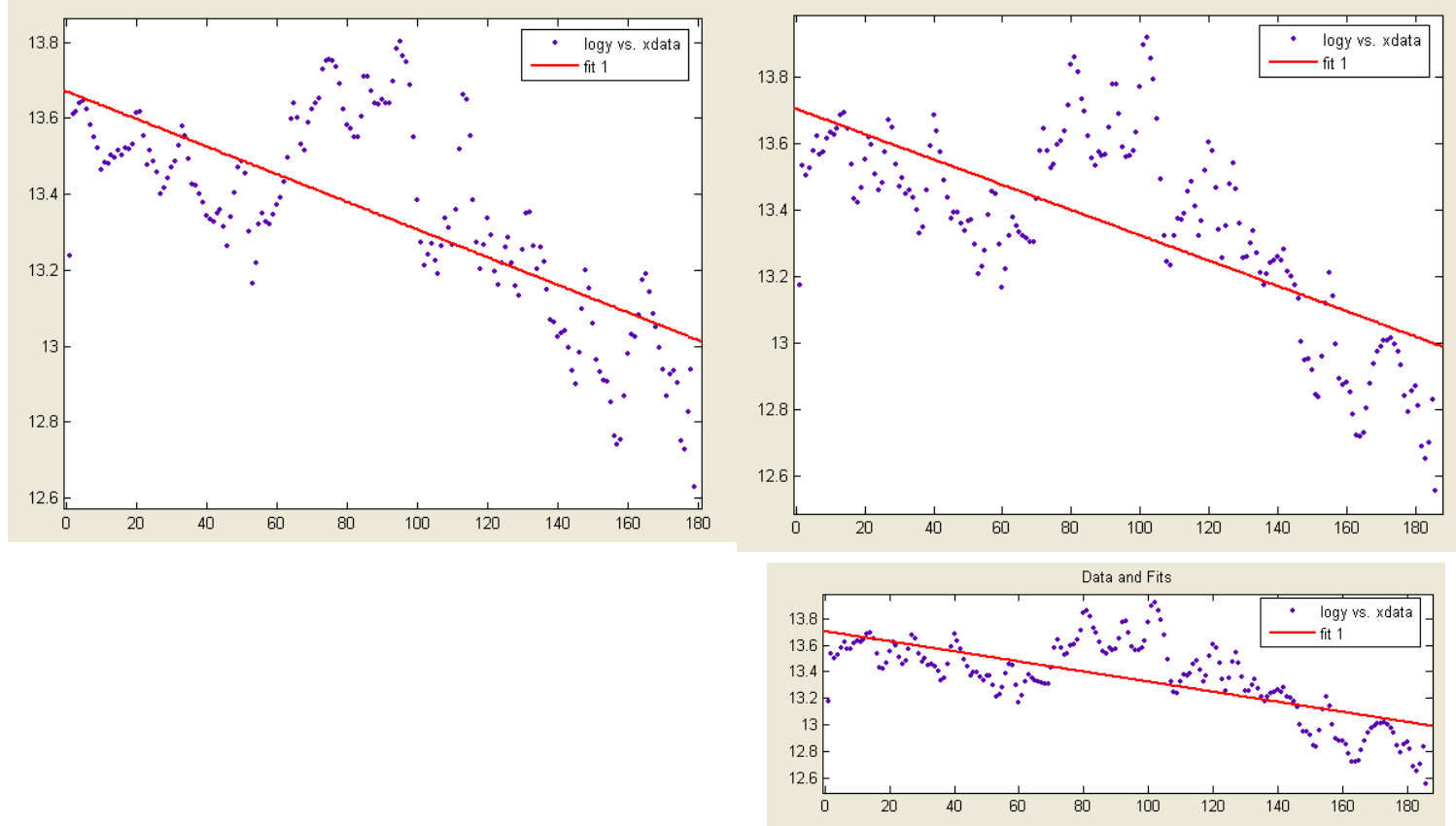

Figure 4.24 Intensity vs pixel distance plot for B634AOA for water and water rinse 

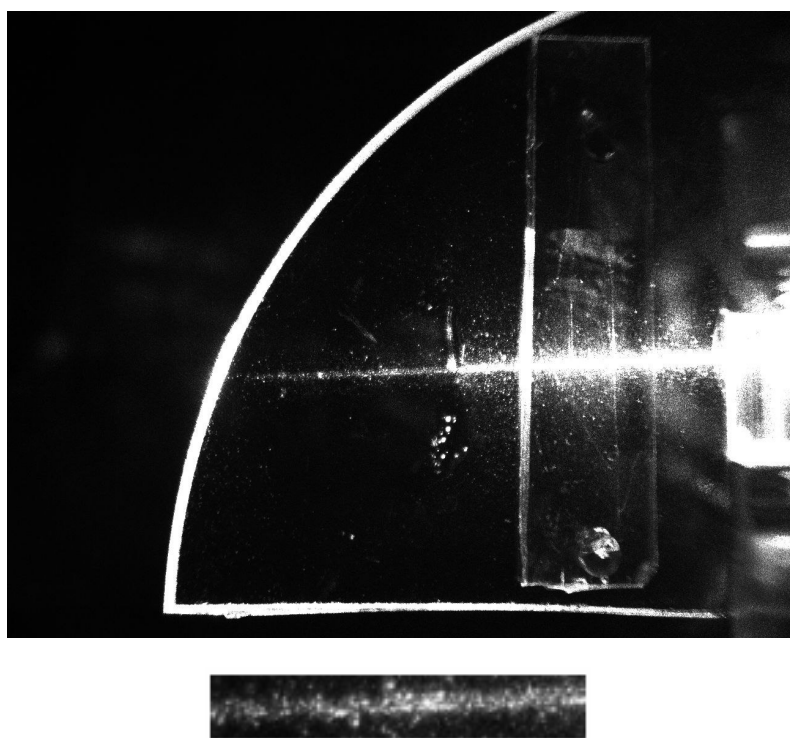

Figure 4.25 B634AOA for exp of 10s with PDMS SU8 2\% sucrose bottom waveguide
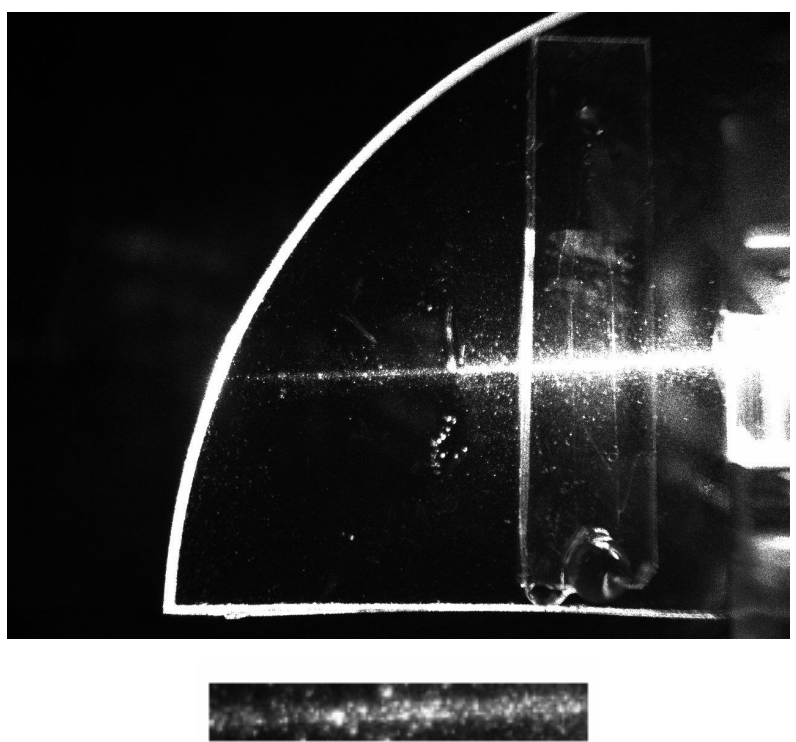

Figure 4.26 B634AOA for exp of 10s with PDMS SU8 2\% sucrose + rinse (9.04) bottom waveguide 

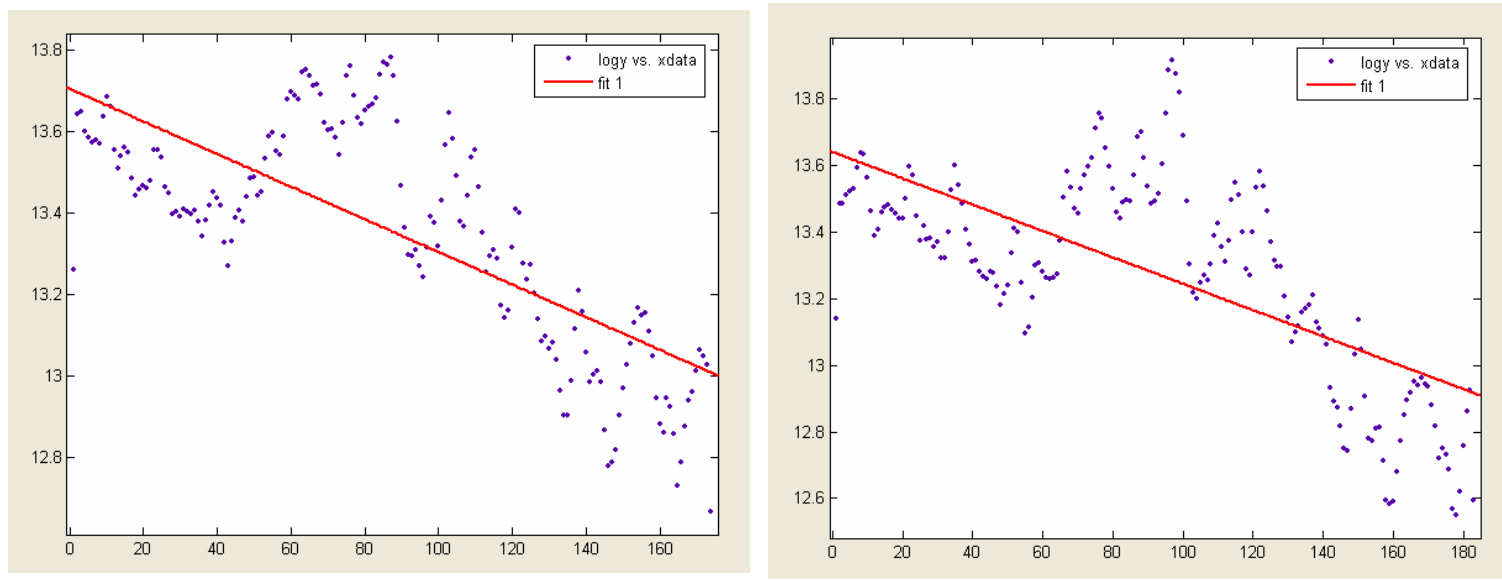

Figure 4.27 Intensity vs pixel distance plot for B634AOA for PDMS SU8 2\% sucrose + rinse (9.04)
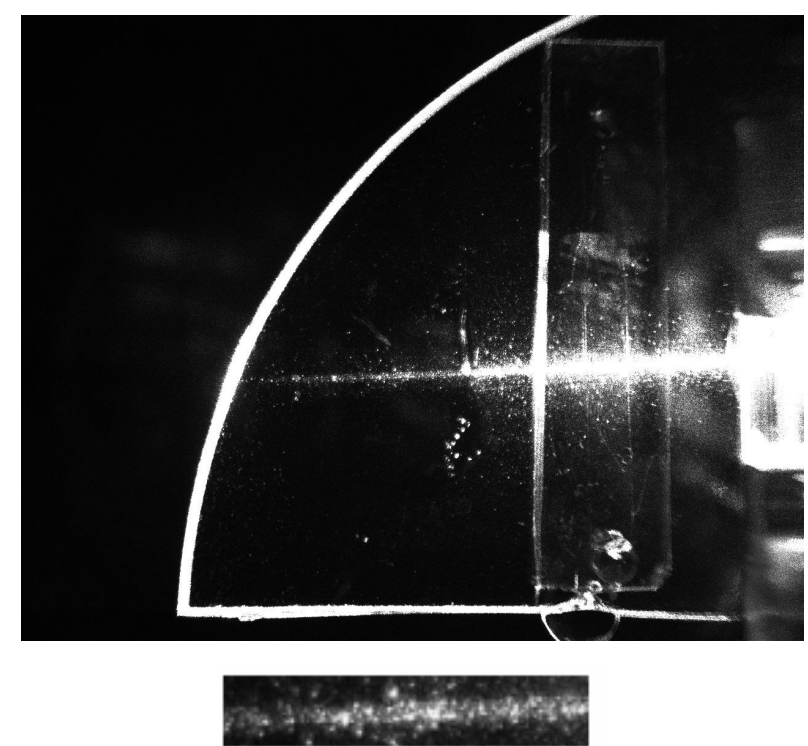

Figure 4.28 B634AOA for exp of 10s with PDMS SU8 2\% sucrose + rinse(9.04) + water

\begin{tabular}{|c|c|c|c|}
\hline Sample & Steps & Loss $(\mathrm{dB} / \mathrm{cm})$ & Error \\
\hline B634AOA & Before experiment & 0.861 & 0.24 \\
\hline & After SU8 & 3.208 & 0.74 \\
\hline & $\begin{array}{c}\text { Water flow of } \\
0.904 \mu 1 / \mathrm{min}\end{array}$ & 4.07 & 0.6 \\
\hline & $\begin{array}{c}\text { Water + rinse } \\
9.04 \mu \mathrm{l} / \mathrm{min}\end{array}$ & 3.953 & 0.54 \\
\hline & Water + rinse + sucrose & 4.479 & 0.6 \\
\hline & $\begin{array}{c}\text { Water + rinse + sucrose } \\
+ \text { rinse }\end{array}$ & 4.426 & 0.69 \\
\hline & $\begin{array}{c}\text { Water + rinse + sucrose } \\
+ \text { rinse + water }\end{array}$ & 4.244 & 0.63 \\
\hline
\end{tabular}

Table 4.5 Optical Propagation Loss Data obtained for each step 
For channel width of $1200 \mu \mathrm{m}$ the response is shown below

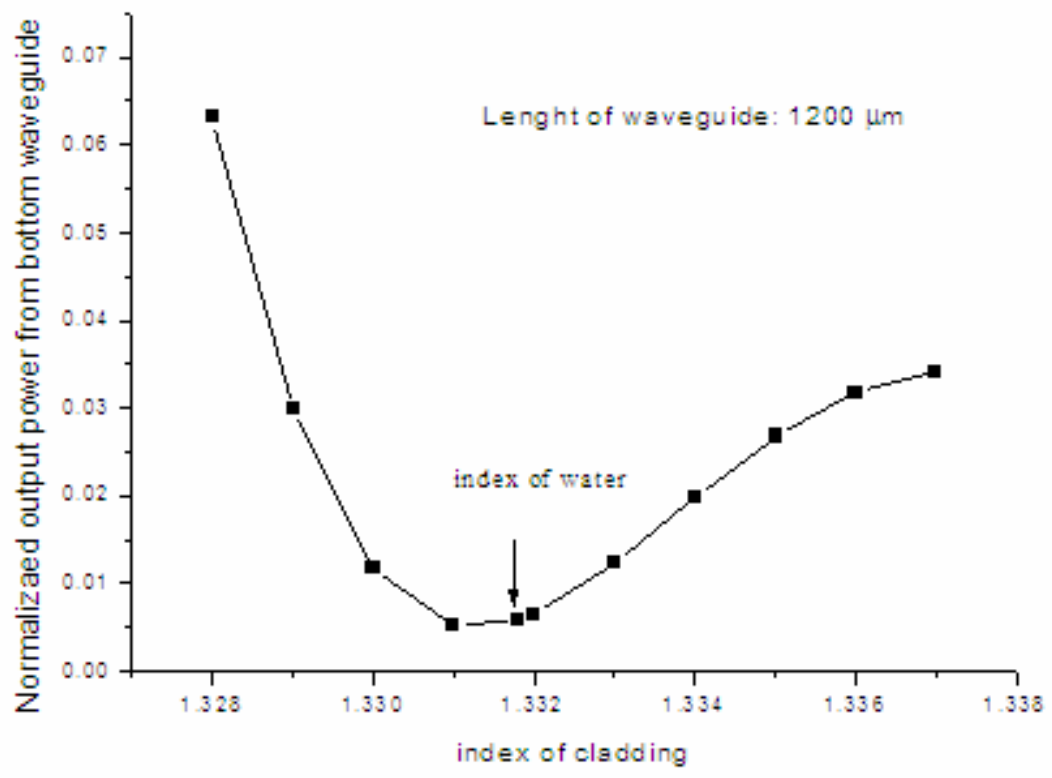

Figure 4.29 The response for normalized output power vs Index of cladding (analyte) $1200 \mu \mathrm{m}$

With the same structure, for channel width of $1275 \mu \mathrm{m}$ the response is shown below which is similar to the ideal structure.

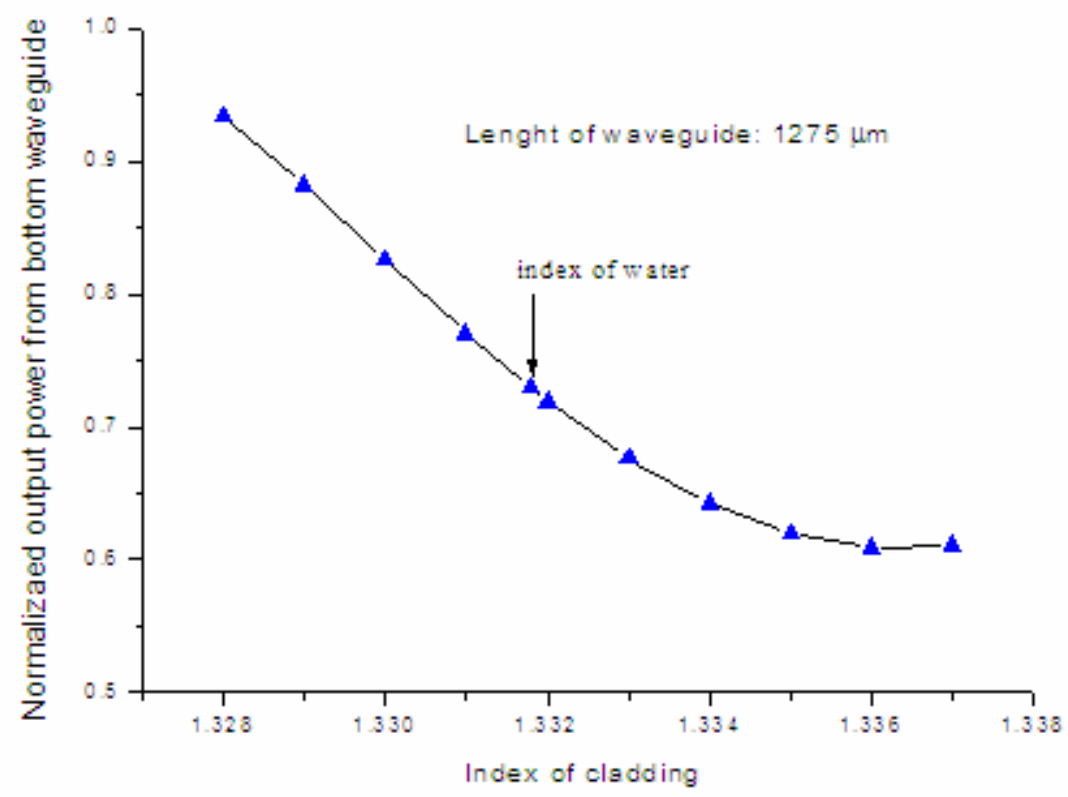

Figure 4.30 The response for normalized output power vs Index of cladding (analyte) $1275 \mu \mathrm{m}$ 


\section{Summary:}

Refractive index of alumina films can be engineered. Alumina films with low losses can be fabricated. Ion source behavior could be explained and it could also be inferred that films deposited with lower electron emitter current donot guide well. Silicon dioxide growth was optimized. Various experiments were performed for depositing silicon dioxide and to study the characteristics of silicon dioxide. $\mathrm{SiO} 2$ from silica source material, switched from Manual to remote, silicon cruicble/ material conditioning. For multi-layer waveguides the model was fixed based on each single layer deposition. We can fabricate samples according to the design. Flow cell experiment were conducted and Analyte (sucrose) detection was performed.

Points to be taken care of :

1. Chamber of Ebeam evaporator should always be clean no contamination.

2. Ion source filaments should always be replaced for every run.

3. Crystal monitors be replaced for every run.

4. Application of SU8 is to be done very carefully.

5. Never use SU8 developer when PDMS is bonded to the alumina surface.

6. PDMS bonding should not have air traps. 


\section{Future Work and Conclusions}

\section{Principal Contributions}

A PDMS based micro fluidic channel has been successfully hybridly integrated with the SPARROW film stack, the bonding was good enough as there was no detachment of the channel from the waveguide surface throughout the experiment. This produces significantly less strain on the sample and doesn't change the prism coupling spot conditions when under test.

Optical loss variation of alumina waveguides as a function of ion beam parameters like drive current and oxygen flow rate has been explored. For the current deposition technique, losses increase with ion drive current and decrease with oxygen flow rate. The reasons for this behavior though are under investigation.

An issue with the ellipsometer measurement and modeling of multi layer films was addressed. A protocol has been developed to characterize the waveguide stack.

A new approach of using SU8 photoresist instead of etching the top waveguide surface was implemented. This avoided the unnecessary surface changes caused due to reactive ion etching the top waveguide to create a window for measurements.

A protocol to conduct the measurements and analyze the data for the actual working device is performed. Experiments conducted on a non-ideal SPARROW structure and the output data for different analytes (such as water and sucrose) were analyzed.

This work enables the next step of fow cell experiments with the SPARROW device to be performed for different index fluids and analytes and the responses to be verified with simulation results.

\section{Future Directions}

The following areas are important future directions, necessary for the subsequent test and evaluation of the SPARROW device.

- The design of the inlet channel needs to be modified in order to eliminate the clogging of the channel with the analyte solution. 
- Capillary tube should be fixed such that deflections in the coupling spot or the coupling are not allowed while the analyte flows.

- Channel width of a non-ideal structure can be engineered in order to obtain a response similar to an ideal structure. 


\section{References}

1. Saraju P. Mohanty, Elias Kougianos, "Biosensors: A tutorial review," IEEE 2006

2. Frieder W. Scheller, Axel Warsinke, Franke F. Bier, Ulla Wolleberger, Wen Jin, Alexander Benkert and Dorothea Pfeiffer, "Biosensors and their applications,"

3. Jerome S. Schultz, "Biosensors," Scientific America, August 1991.

4. J. H. Jerman and D. J. Clift, "Miniature Fabry-Pérot interferometers micromachined in silicon for use in optical fiber WDM systems," in Tech. Dig. Transducers, 1991, pp. 372375.

5. Ryszard M. Lec, Peter A. Lewin, “Acoustic wave biosensors,” IEEE, 1998.

6. J. Janata, "Principles of Chemical Sensors" New York: Plenum, 1989.

7. Andreas Hierlemann, Oliver brand, Christoph Hagleitner, and Henry Baltes, Fellow, IEEE, "Microfabrication Techniques for Chemical/Biosensors" IEEE Vol. 91, No.6 June 2003

8. J. W. Grate, S. J. Martin, and R. M. White, "Acoustic wave microsensors," in Sensors Update, H. Baltes, W. Gopel, and J. Hesse, Eds. Weinheim, Germany: VCH, 1996, vol. 2, pp. 37-83

9. M. J. Vellekoop, G. W. Lubking, P. M. Sarro, and A. Venema, "Integrated-circuitcompatible design and technology of acoustic-wave-based microsensors," Sens. Actuators A, pp. 249-263, 1994.

10. S. W. Wenzel and R. M. White, "Flexural plate wave gravimetric chemical sensors," Sens. Actuators A, vol.21-23, pp. 700-703, 1990.

11. A. W. Wang, R. Kirwan, R. M. White, and R. L. Ceriani, "A silicon based ultrasonic immunoassay for detection of breast cancer," Sens. Actuators B, vol. 49, pp. 13-21, 1998. 12. A. Hierlemann, D. Lange, C. Hagleitner, N. Kerness, A. Koll, O. Brand, and H. Baltes, "Application-specific sensor systems based on CMOS chemical microsensors," Sens. Actuators B, vol.70, pp. 2-11, 2000.

13. D. Lange, C. Hagleitner, A. Hierlemann, O. Brand, and H. Baltes, "Complementary metal oxide semiconductor cantilever array on a single chip: Mass-sensitive detection of volatile oraganic compounds," Anal. Chem., vol.74, pp. 3084-3095, 2002. 
14. A. W. van Herwaarden, P. M. Sarro, "Thermal sensors based on the Seeback effect," Sens. Actuators, vol.10, pp. 321-346, 1986.

15. S. Semancik and R. E. Cavicchi, "Kinetically controlled chemical sensing using micromachined structures," Accounts Chem. Res., vol. 31, pp. 279-287, 1998.

16. I.Lundstrom, S. Shivaraman, C. Svensson, and L. Lundkvist, "Hdrogen-sensitive MOS field effect transistor,” Appl. Phys. Lett., vol.43, pp. 55-57, 1975.

17. O. S. Wolfbeis, G. E. Boisde, and G. Gauglitz, "Optochemical sensors," in Sensors Update, W. Göpel, J. Hesse, and J. N. Zemel, Eds. Weinheim, Germany: VCHVerlagsgesellschaft, 1991, vol.2, pp. 573-646.

18. G. Gauglitz, "Opto-chemical and opto-immuno sensors," in Sensors Update, H. Baltes, W. Göpel, and J. Hesse, Eds. Weinheim, Germany: VCH-Verlagsgesellschaft, 1996, vol.1, pp. 1-49.

19. Lars Lading, Lars Bjarne Nielsen, Torben Sevel, "15.1: Comparing Biosensors," IEEE, 2002.

20. W. Lukosz, "Integrated optical chemical and direct biochemical sensors," Sens. Actuators B, vol.29, pp.37-50, 1995.

21. Naveen Paturi, Chinnayya R. Vemuri, Dimitris Korakakis, Lawrence A. Hornak., (2005, Dec), "Analysis of cluster defects in Photonic Crystals for biosensor applications", Proceedings of SPIE Vol. 6005, pp 146.

22. Evanescent Waves, Carnegie Mellon Univeristy http://www.andrew.cmu.edu/user/ dcprieve/Evanescent\%20waves.htm.

23. D. Lloyd, L. A. Hornak, S. Pathak, D Morton, and I. Stevenson, "Application of Ion Beam Assisted Thin Film Deposition Techniques to the Fabrication of a Biosensor Chip With Fieldability Potential for Important Biohazard Detection Applications" 47th Annual Technical Conference Proceedings, Soc. of Vac. Coaters, ISSN 0737-5921 (2004).

24. T. S. Eriksson, A. Hjortsberg, G.A. Niklasson, and C. G. Granqvist, "Infrared optical properties of evaporated alumina films" Applied Optics, 20 (15), 2742 (1981).

25. Joseph Palais, "Fiber Optic Communicaitons" 5th Edition, Prentice Hall Publications.

26. Bahaa E. A. Saleh, Malvin Carl Teich, "Fundamentals of Photonics". 
27. P. K. Tien and R.Ulrich, Bell Telephone Laboratories, New Jersey, "Theory of PrismFilm Coupler and Thin-Film Light Guides," Journal of the optical society of America vol. 60 (10). 1970.

28. Donald L. Lee, "Electromagnetic principles of Integrated Optics"

29. Cornell T., (2005), "Fabrication of Resonant Optical Waveguide Biosensors using Electron Gun Depositions", Masters Thesis, West Viriginia University.

30. P. K. Samudrala, "Alumina Waveguide Characterization and SPARROW Biosensor Modeling", Master Thesis, West Virginia University.

31. J. R. Nightingale, P. Poloju, T. Cornell, P. Samudrala, L. A. Hornak, and D. Korakakis, "Reactive Deposition of Dielectrics by Ion Beam Assisted E-beam Evaporation," Lane Department of Computer Science and Electrical Engineering, West Virginia University.

32. P. Poloju, P. K. Samudrala, J. R. Nightingale, D. Korakakis, L. A. Hornak, "Characterization of Alumina Optical Waveguides Grown by Ion Beam Assisted Deposition for SPARROW Biosensors," MRS Fall 2006 Conference, Boston.

33. J. R. Nightingale, P. Poloju, D. Korakakis, and L. A. Hornak, "Application of Ion Beam Assisted Deposition and Prism Coupling Characterization Techniques for a Resonant Optical Waveguide Biosensor," Nanofab Conference Spring 2007, Carnegie Mellon University.

34. J. E. Mahan, Physical Vapor Deposition of Thin Films (John Wiley \& Sons, Inc., New York, 2000).

35. Nano SU8 25 Specifications Sheet.

36. Shantanu Bhattacharya, Aridom Datta, Jordan M Berg and Shubhra Gangopadhyay, (2005, June), "Studies on Surface Wettability of Poly (Dimethyl) Siloxane (PDMS) and Glass Under Oxygen-Plasma Treatment and Correlation With Bond Strength," J.Microelectromechanical Systems, Vol. 14, No 3.

37. Optiwave Documention for OptiBPM 7.0, Optiwave Corporation, Ottawa, ON, Canada. 


\section{APPENDIX A}

\section{Prism coupler}

The basic coupling measurements of effective indices and modes in a thin film on a substrate is detailed in this section.

1. Turn on the laser and the interface box followed by the software Metricon 2010 Version 1.61.30 DIO. The user will be prompted to reference the table before starting the measurements. User can choose to perform a manual or auto referencing procedure although for most cases auto referencing should suffice.

2. Figures below shows the initial screen that opens as the software is turned on. The buttons below shows the various options that can be invoked. Clicking the parameters button will open up a window where initial measurement parameters can be specified such as substrate index, prism index, scan parameters etc.

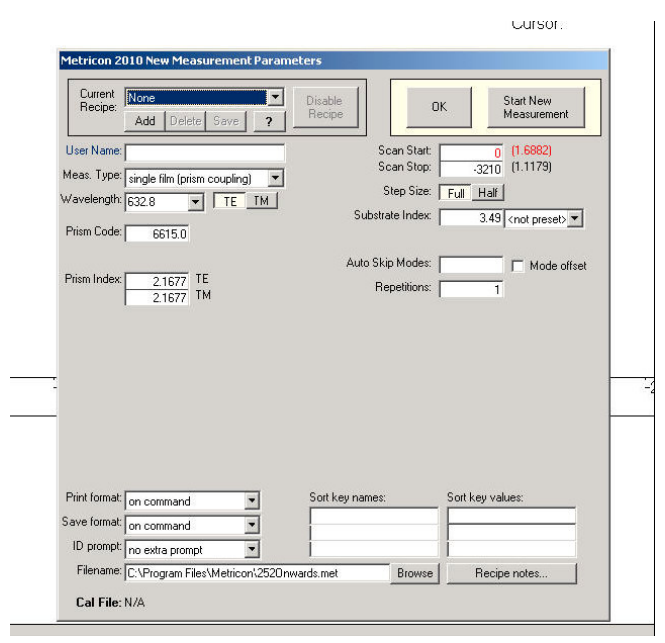



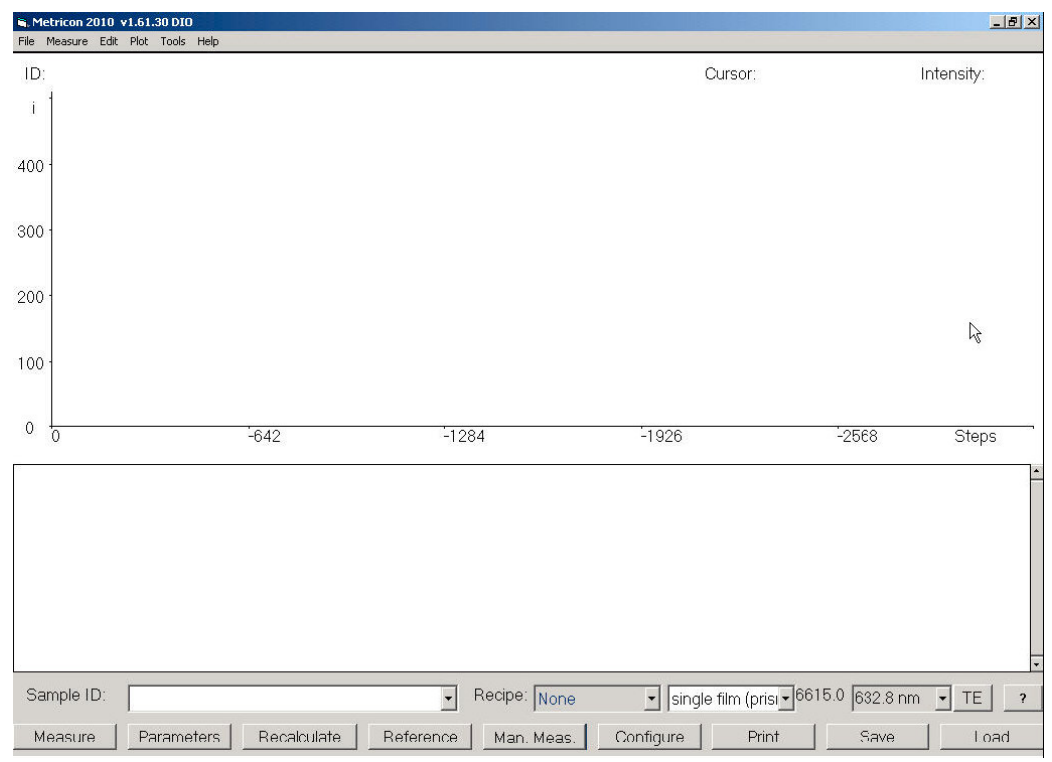

3. The screen below shows the actual measurement screen invoked by clicking on Manual measurements on the home screen. Either auto scan can be performed or a manual scan can be done using the control pod. Also polarization can be changed depending on the measurement type desired. After all the parameters are specified the sample should be clamped against the prism using the plunger and the laser should be aligned with the coupling spot.

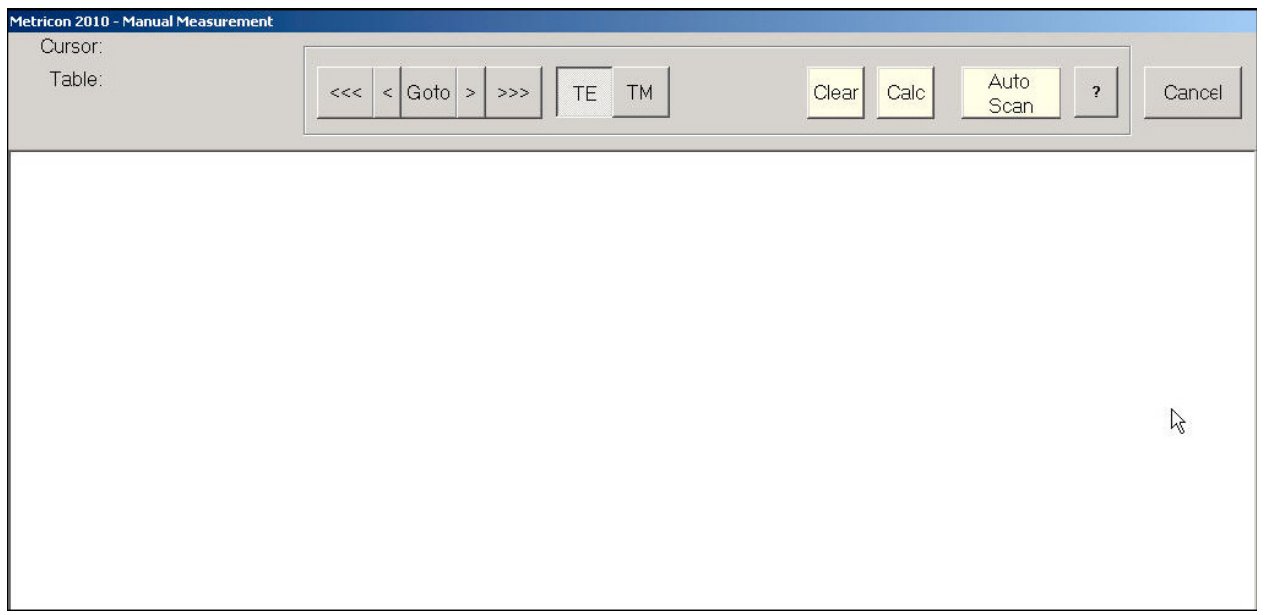

4. After performing the measurements the software automatically picks up modes and calculates the thickness and index of the film along with the standard deviation in the measurements provided more than two modes are found. An example is shown in the picture below. Also using recalculate button on the home screen the values can be recalculated by adding or deleting the modes if the 
software is unable to pick up modes correctly. Also other advanced feature such as mode offset and auto skip can be invoked using this screen.

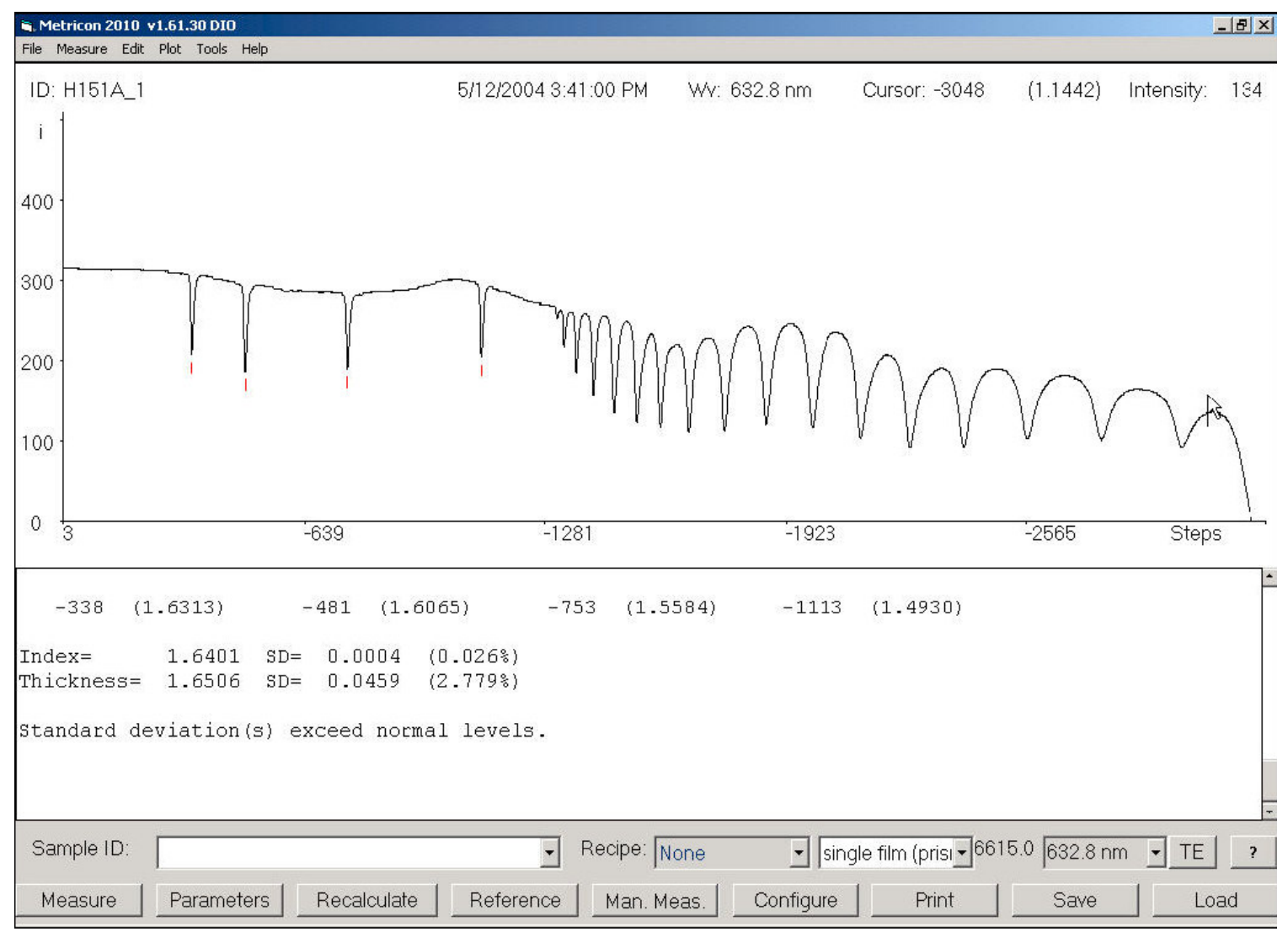

5. Viewing the coupling spot: If the coupling spot is not visible, cleaning the prism and the sample using methanol and cleanroom wipe helps for obtaining a good coupling spot on contact. We can increase the pressure of the plunger in the increments of 2-3 psi. Do not increase the pressure over 55 psi. 


\section{APPENDIX B}

\section{Loss Measurements using Matlab Program and Prism coupler [reference Pavan's thesis] :}

We have developed a method for computing the loss of the waveguides using prism coupler and Matlab code. When light is coupled into the waveguide using prism coupler, a guiding streak is imaged with a CCD camera and is processed in Matlab to compute the loss of the waveguide. The process for the same is detailed in this section.

- Mount the sample onto the Prism coupler and need to reference it if concerned with the beta values of the film.

- After calculating the effective indexes, the photo detector is removed the CCD camera is placed focusing on the regions we like to measure.

- A graph paper is pasted on the back side of the sample and take measurement with light switched on; this image can be used as a reference for pixel to distance conversion.

- Without changing the focus of the camera take the images for different exposure times.

\section{Matlab Code:}

Copy the file latest.m [30] and paste it in the folder containing the images. Open the file from Matlab and input the name of the image you want to measure the losses for. The code computes the average of the pixels around each point.

- First we need to align the image as straight as possible since the image taken with graph paper may not be aligned properly. Code has already been written to align the graph but the user has to identify two points that needs to be in a straight line.

- Compute the distance between the two points selected. Hence it would be easier if we select the two points between whose distance is already known. We can select any two points on the graph paper and the distance between them can be obtained in terms of pixels.

- The pixel to distance ratio would remain constant as long as the focus and the position of the camera does not change. 
- The next step is to select the image whose loss is to be determined.

- A dark image should also be taken at the same time when the original images were taken to include the effect of dark current of the CCD camera.

- The dark image is subtracted from the original image which takes into account the effect of background.

- The new image has to be used for computing the losses.

- A code has been written to select a block of image from the given image. Hence the user can select the intended portion of analysis by using the mouse.

- Once a portion of the image is selected, it undergoes through a series of filters. The first filter is the scattering removal filter. A code has been written to identify the scattered pixels based on the intensity of the pixels. If the intensity of a pixel is very much larger than the average of the surrounding pixels then it is considered to be a scattered pixel and it replaced by an average value of the surrounding.

- After an image is selected and filtering is done (latter is user's choice) the column intensities along each row are computed and are stored in a 1D matrix.

- Logarithm of the matrix is taken and 'cftool' (Curve fitting Tool) is employed in Matlab to fit the selected points. It gives the slope along with $95 \%$ confidence levels too. 

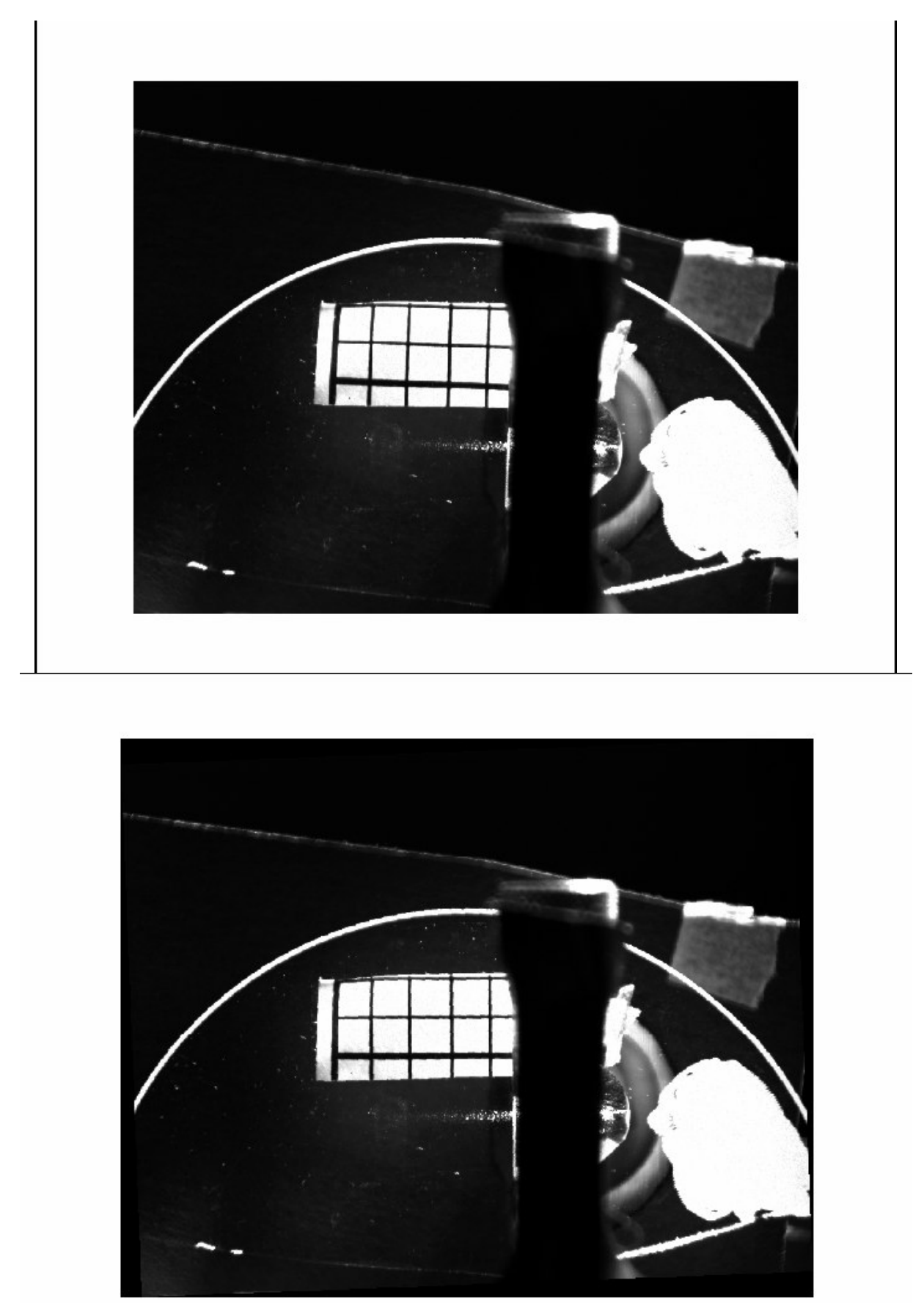

The above process is repeated a couple of times and at different regions and exposure times. The average of the losses is computed and is taken as the loss of the sample. It is possible that you would see different losses at different exposure times and that is because of the gamma factor of the camera. This means that the whole area exposed is not uniformly sensitive and hence a gamma correction is needed. Usually the 
gamma factor is assumed to vary linearly in our working range and hence is taken to be unity.

Images as per the steps said above:

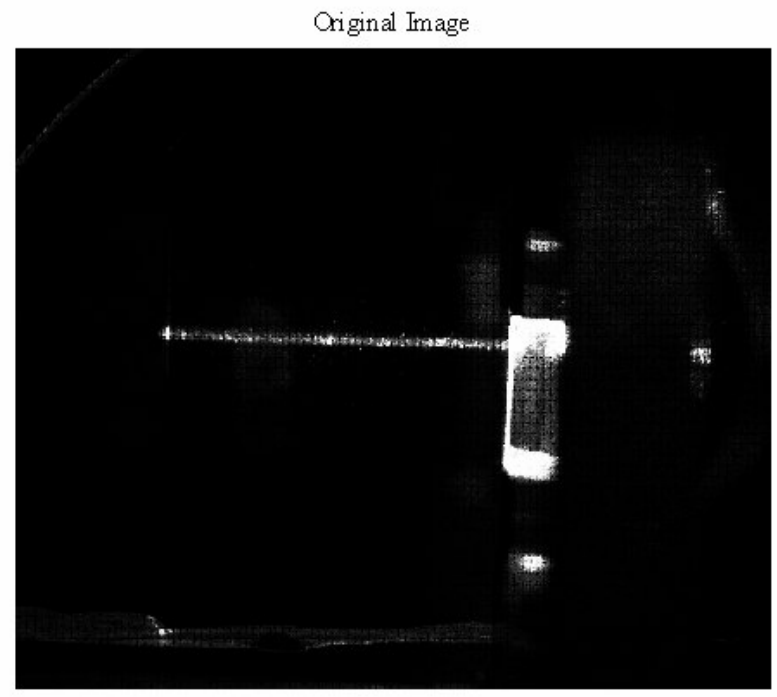

Original Image with area to be Cropped

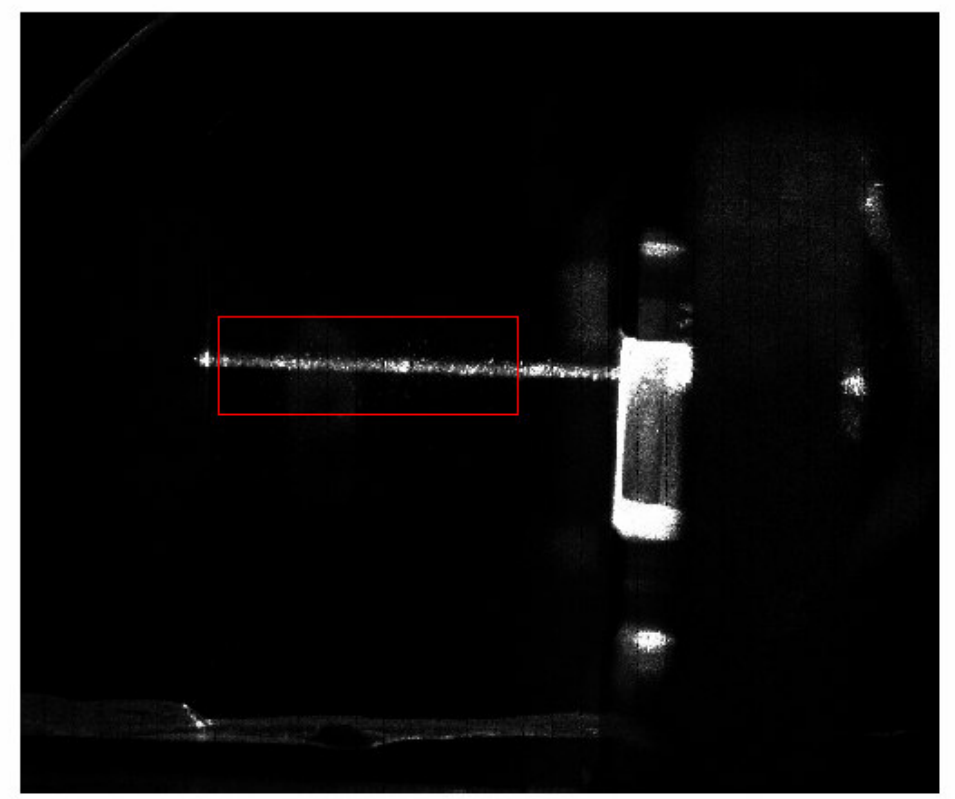

Compressed Original size image 


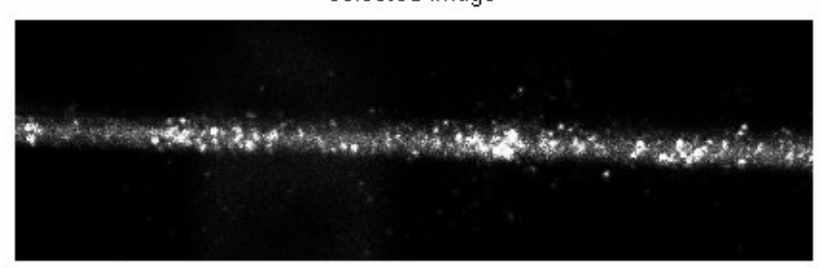

Image after removing the scattering points but before blurring

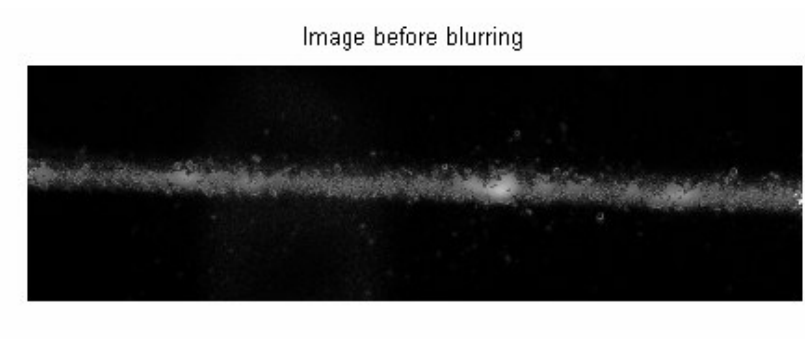

Image after blurring the original image (This step is optional and can be removed if the image after scattering is good. If the original image has lot of scattering points and cannot be removed from the scattering filter then this step can be used as it averages all the pixels and hence there wouldn't be any error in the measurement)

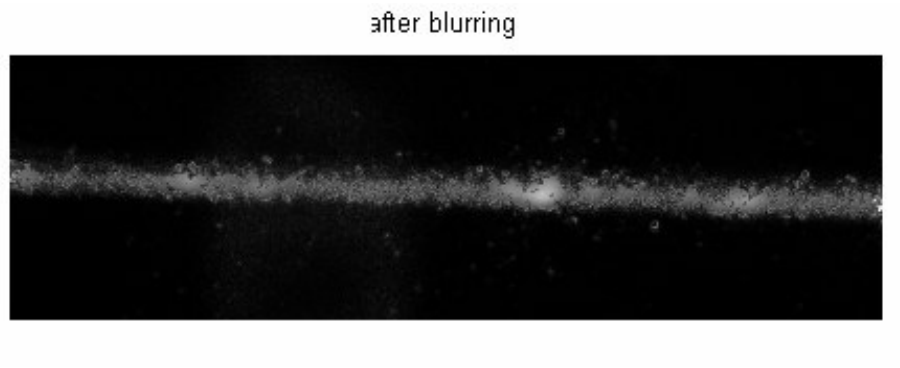

This plot has the logarithm of intensities of the given image. From Figureure 6 we observe that the region good for measuring losses is till 200 pixels even though there are little disturbances due to the scattering. But after 200 pixels there is enormous effect of scattering that cannot be removed by any filter and hence the user has to exclude those points using the curve fitting software. 


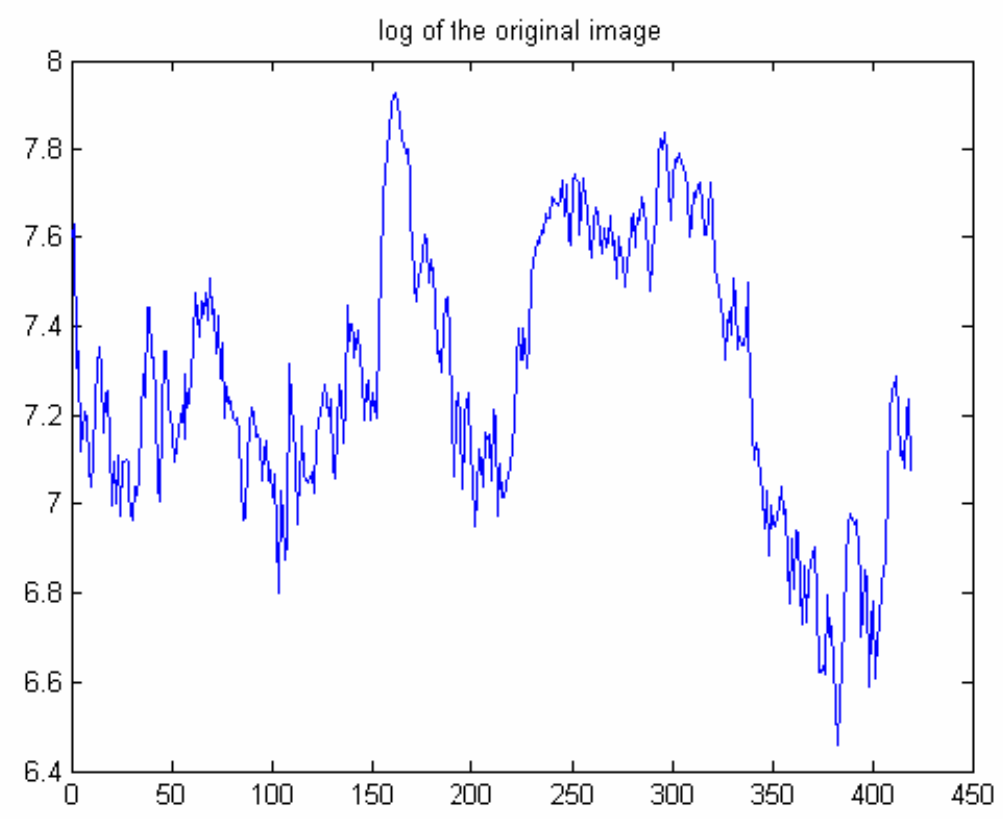

After excluding the unnecessary pixels the graph looks like the below Figureure where the shaded region corresponds to the pixels that have been neglected for loss computation.

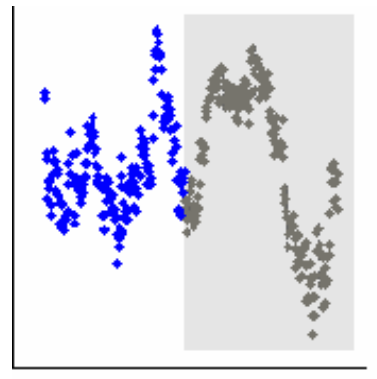

The following is the image we got after employing curve fitting for the selected region.

The calculation has been done for the necessary region though it cannot be seen in the image below. The slope of the line can be obtained in the fitting window. The slope of the line can be used to calculate the losses of the waveguide by using an empirical formula $4.3^{*} \alpha$ where $\alpha$ is the loss per $\mathrm{cm}$. The slope in this window gives the slope per pixel. This has to be converted to the scale of $\mathrm{cm}$ using the pixel to distance ratio. Calculation of that ratio is described in the previous section. 


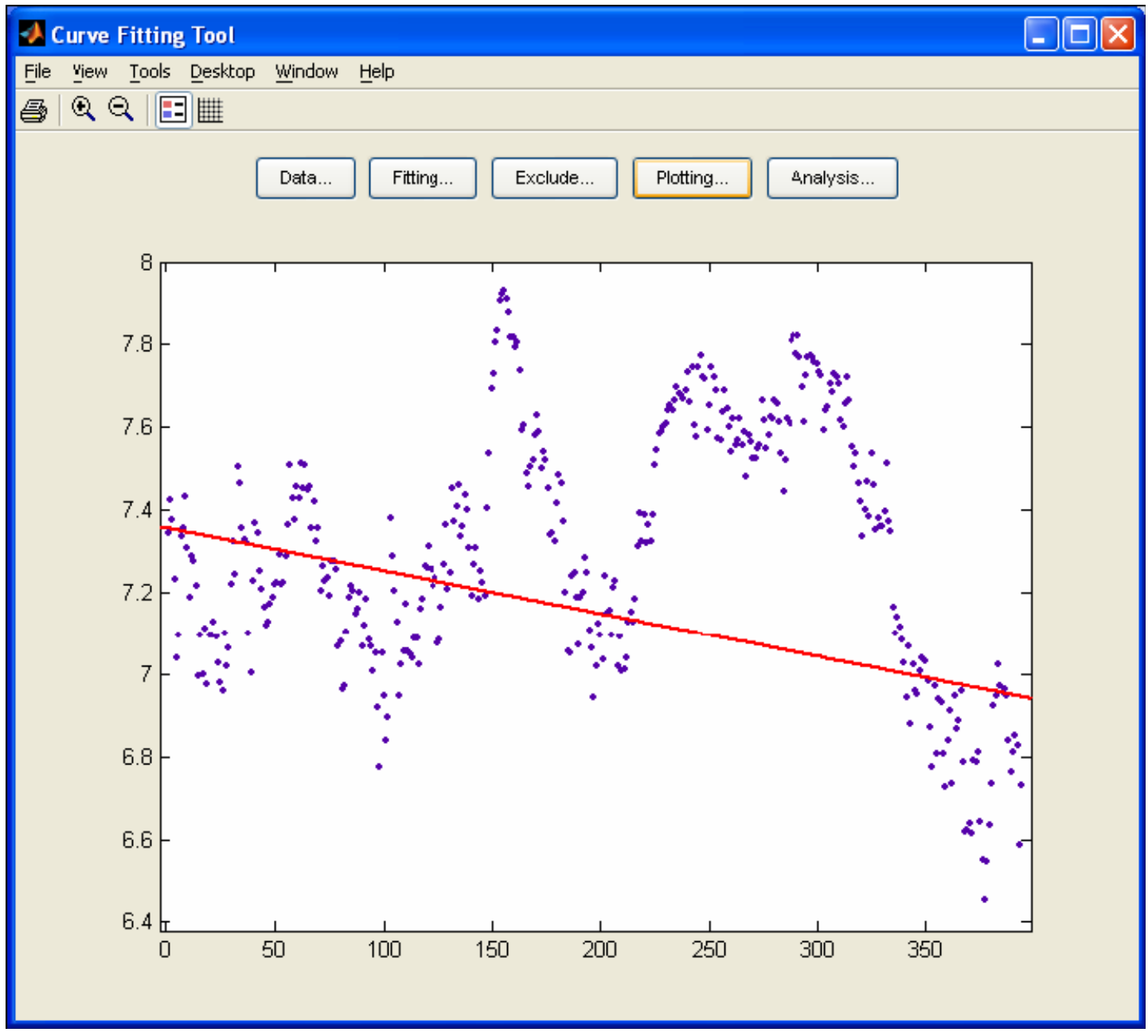




\section{APPENDIX C}

\section{OPTIBPM 7.0 Simulations and Modeling Parameters}

\section{Procedure for Execution of Simulations}

We use Mode solver 2D for calculating effective refractive indices and thereby beta values. OPTIBPM 7.0 has some assumptions and would not give correct modal indices. Compare them with the values obtained from prism coupler.

The input waveform for the waveguide stack if done from the BPM tool would not give the correct modal indices as it assumes the wafer on either side of the waveguide. The best method is to get the input wave using 2D Mode solver. We need to have same mesh size and the wafer size when we do this.

To vary the thicknesses of the waveguides or wavelength of operation we can change the script, but we have to incorporate the shifting of the waveguide offset due to the change in the thicknesses. (Not necessary if parameter is wavelength).

Path Monitor and slice selector options can prove very helpful to observe the field in the waveguides.

Using the parameters and varying them as a series could prove helpful in determining the behavior of the device when the thickness and indexes are off.

\section{Input types in OPTIBPM 8:}

There are numerous ways of assigning input to the designed structure. The general input files that are found in the software are Modal, Gaussian, Rectangular and through File. An input wave is selected based on the excitation given in the real measurement. The actual device is excited with a prism coupler at certain modes we can use the modal input for performing our simulations, the assumptions for using this input are detailed in the next paragraph. With the modal input excitation a waveguide is selected from the design and mode is calculated assuming that the cladding and substrate layer indices are same for the wafer. In all practical uses even a single layer waveguide based device would also be an asymmetric waveguide and the input if used through this way will always be calculated for a symmetric waveguide and this would work fine for single waveguide designs but for multilayer films having two or more waveguides it is 
good to used a structure designed in 2D Mode Solver and calculate the modes. After the calculations the file is saved as an .f2d file and can be used as an input to the structure for simulations. The number of mesh points and the wafer width has to be the same in " $2 \mathrm{~d}$ Mode Solver" and OPTIBPM 8 to perform the simulations. Since we are using input file separately, file checkbox has to be selected for this type of calculations.

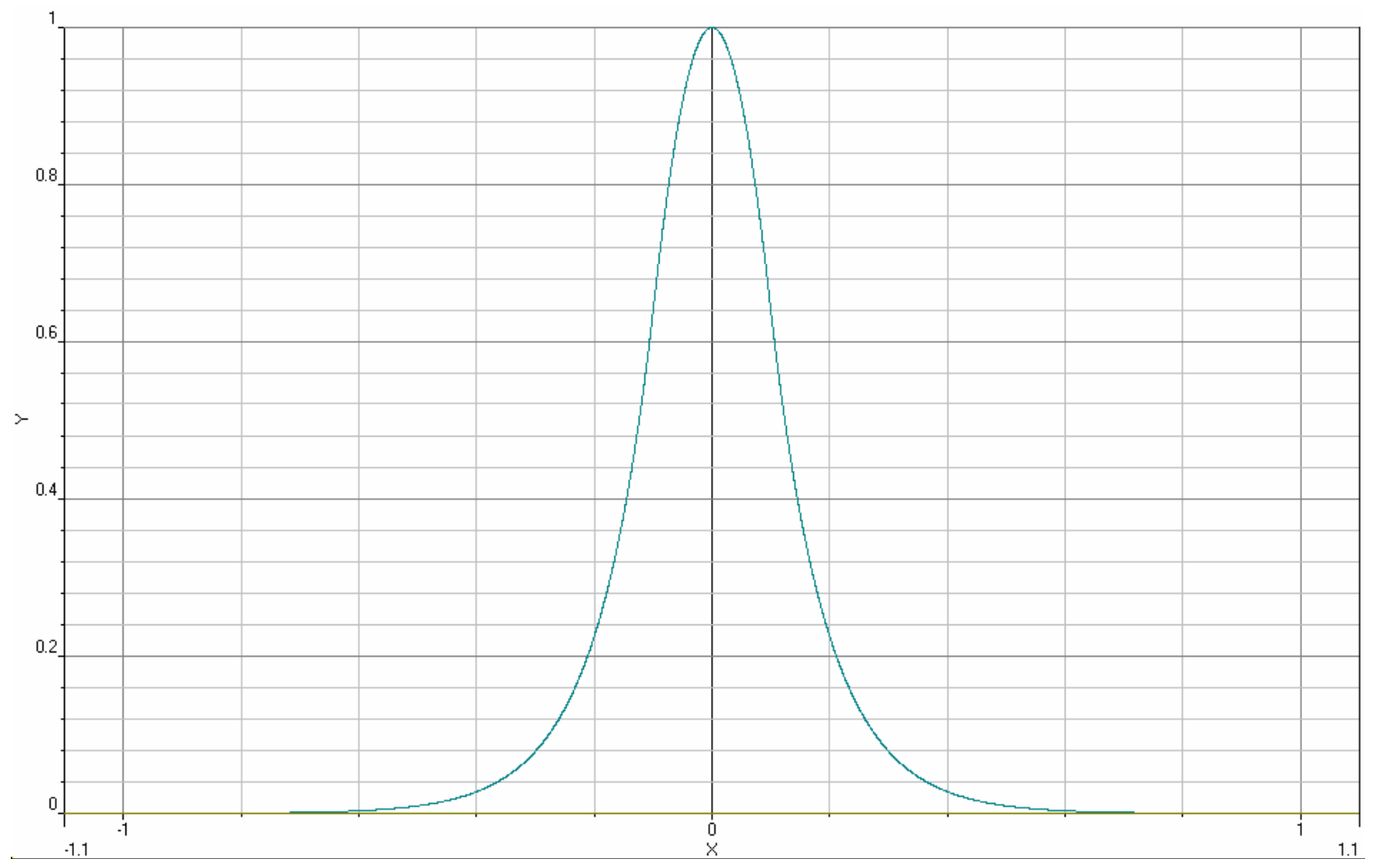

\section{Path Monitor and Power at the ends of Waveguide:}

'Path monitor' can be used to see the variation of power in the designed waveguides and 'Power in Output Waveguide' can be selected to observe the powers at the ends of the waveguide. This can prove helpful if the variations are less and which cannot be observed visually.

\section{Process for computing a simulation for structure:}

Figure 2 is the structure for which the TE simulation would be performed. Light would be input in the bottom waveguide and optical field intensity would be observed. The same structure is designed in " $2 \mathrm{D}$ Mode solver" to obtain the input wave. But for the input wave the top layer is assumed to be air since during prism coupling the modal input would be due to air. 
Figure 3 shows the input wave obtained using mode solver with the top layer index of air. Since in BPM simulation tool the input can be given only at the center of the wafer the final thicknesses for water and Borofloat are to be found. The mesh was assumed to be 8000 . Assuming the thickness of water to be 1 micron the final thickness of Borofloat would be 2.294 microns $(1+0.194+1.1+0.144 / 2)$. And the whole thickness of the wafer is 4.732 micron. These values are used in the BPM simulator with the same number of mesh points.

\begin{tabular}{ll|}
\hline Water & $\mathrm{N}=1.3318$ \\
\hline Alumina & $\mathrm{N}=1.659 \mathrm{~d}=194 \mathrm{~nm}$ \\
\hline Silica & $\mathrm{N}=1.457 \mathrm{~d}=1100 \mathrm{~nm}$ \\
\hline Alumina & $\mathrm{N}=1.659 \mathrm{~d}=144 \mathrm{~nm}$ \\
\hline Borofloat & $\mathrm{N}=1.4701$ \\
\hline
\end{tabular}

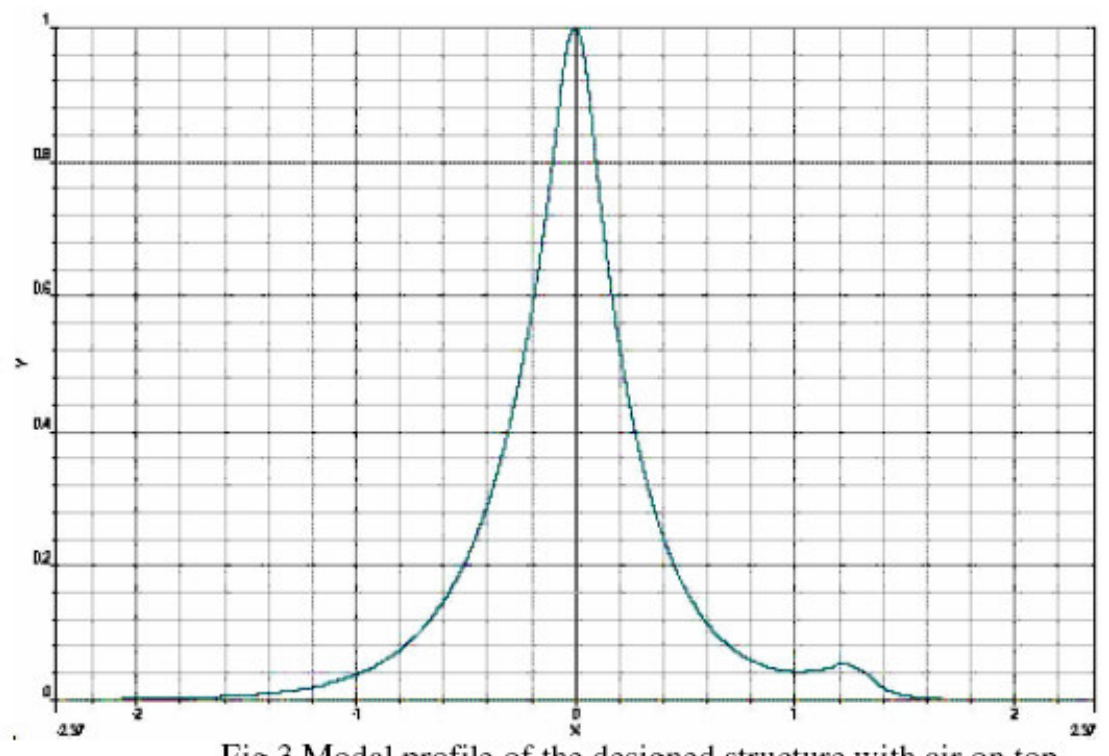

Fig 3 Modal profile of the designed structure with air on top

In the profiles and materials dialog box define the materials that are going to be used in the design under dielectrics tab. In the channels tab select each material that is going to be used in the design. In 3D simulations these channels can be used to define certain shapes. Since we are concerned with only 2D simulations we ignore the 3D parameters. A screen shot of defined materials and channels is shown in Figure 4. 


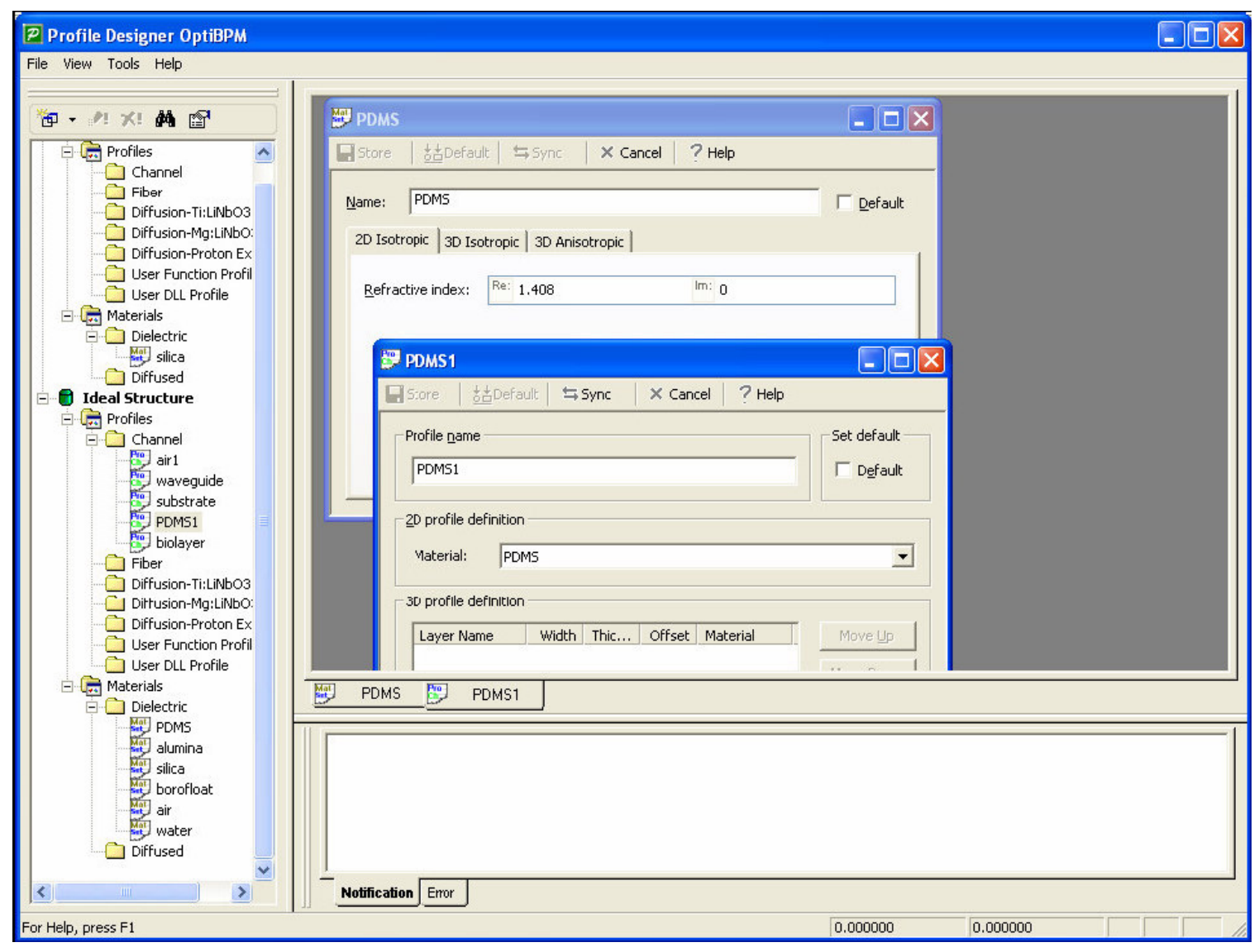

Figure 4 Profile Designer Window

After the desired materials are defined the wafer properties dialog box can be filled. Select the wafer material from the designed channels and the width of wafer would be the calculated one from mode solver. The length of the wafer depends on the interaction length we choose to perform the simulation for. Default waveguide generally can be chosen any of the material as it can be changed in the design layout window. We need to hit "OK" tab once the wafer properties dialog box is filled, it would takes us to the layout window. We can design the linear waveguides from "Draw" tab and the length and width of the waveguides are to be specified as per the design. It is to be noted that the waveguide is laid symmetric such that the location specified would be the axis of the waveguide. From the "simulation" menu "simulation parameter" is selected. Mesh points and wavelength are selected as per the mode solver.

Input plane is selected from draw menu and the "file" option is selected for giving input from another file. File obtained from mode solver is selected. Once the input is selected the next step is to perform the simulation. Extra features like path monitor, output power in waveguides can be selected depending upon the parameter of interest. 